\title{
Gaia Data Release 1
}

\section{Testing parallaxes with local Cepheids and RR Lyrae stars ${ }^{\star}$}

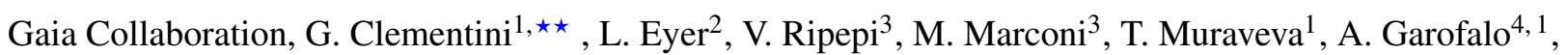
L. M. Sarro ${ }^{5}$, M. Palmer ${ }^{6}$, X. Luri $^{6}$, R. Molinaro ${ }^{3}$, L. Rimoldini ${ }^{7}$, L. Szabados ${ }^{8}$, I. Musella ${ }^{3}$, R. I. Anderson ${ }^{9,2}$, T. Prusti ${ }^{10}$, J. H. J. de Bruijne ${ }^{10}$, A. G. A. Brown ${ }^{11}$, A. Vallenari ${ }^{12}$, C. Babusiaux ${ }^{13}$, C. A. L. Bailer-Jones ${ }^{14}$, U. Bastian ${ }^{15}$, M. Biermann ${ }^{15}$, D. W. Evans ${ }^{16}$, F. Jansen ${ }^{17}$, C. Jordi ${ }^{6}$, S. A. Klioner ${ }^{18}$, U. Lammers ${ }^{19}$, L. Lindegren ${ }^{20}$, F. Mignard $^{21}$, C. Panem ${ }^{22}$, D. Pourbaix ${ }^{23,24}$, S. Randich ${ }^{25}$, P. Sartoretti ${ }^{13}$, H. I. Siddiqui ${ }^{26}$, C. Soubiran ${ }^{27}$, V. Valette ${ }^{22}$, F. van Leeuwen ${ }^{16}$, N. A. Walton ${ }^{16}$, C. Aerts ${ }^{28,29}$, F. Arenou ${ }^{13}$, M. Cropper $^{30}$, R. Drimmel ${ }^{31}$, E. Høg ${ }^{32}$, D. Katz ${ }^{13}$, M. G. Lattanzi ${ }^{31}$, W. O’Mullane ${ }^{19}$, E. K. Grebel ${ }^{15}$, A. D. Holland ${ }^{33}$, C. $\mathrm{Huc}^{22}$, X. Passot ${ }^{22}$, M. Perryman ${ }^{10}$,

L. Bramante ${ }^{34}$, C. Cacciari ${ }^{1}$, J. Castañeda ${ }^{6}$, L. Chaoul $^{22}$, N. Cheek ${ }^{35}$, F. De Angeli ${ }^{16}$, C. Fabricius ${ }^{6}$, R. Guerra ${ }^{19}$, J. Hernández ${ }^{19}$, A. Jean-Antoine-Piccolo ${ }^{22}$, E. Masana ${ }^{6}$, R. Messineo ${ }^{34}$, N. Mowlavi ${ }^{2}$, K. Nienartowicz ${ }^{7}$,

D. Ordóñez-Blanco ${ }^{7}$, P. Panuzzo ${ }^{13}$, J. Portell ${ }^{6}$, P.J. Richards ${ }^{36}$, M. Riello ${ }^{16}$, G.M. Seabroke ${ }^{30}$, P. Tanga ${ }^{21}$,

F. Thévenin ${ }^{21}$, J. Torra ${ }^{6}$, S.G. Els ${ }^{37,15}$, G. Gracia-Abril ${ }^{37,6}$, G. Comoretto ${ }^{26}$, M. Garcia-Reinaldos ${ }^{19}$, T. Lock ${ }^{19}$, E. Mercier ${ }^{37,15}$, M. Altmann ${ }^{15,38}$, R. Andrae ${ }^{14}$, T. L. Astraatmadja ${ }^{14}$, I. Bellas-Velidis ${ }^{39}$, K. Benson ${ }^{30}$, J. Berthier ${ }^{40}$, R. Blomme ${ }^{41}$, G. Busso ${ }^{16}$, B. Carry ${ }^{21,40}$, A. Cellino ${ }^{31}$, S. Cowel1 ${ }^{16}$, O. Creevey ${ }^{21,42}$, J. Cuypers ${ }^{41}$, M. Davidson ${ }^{43}$, J. De Ridder ${ }^{28}$, A. de Torres ${ }^{44}$, L. Delchambre ${ }^{45}$, A. Dell'Oro ${ }^{25}$, C. Ducourant ${ }^{27}$, Y. Frémat ${ }^{41}$, M. García-Torres ${ }^{46}$, E. Gosset ${ }^{45,24}$, J.-L. Halbwachs ${ }^{47}$, N. C. Hambly ${ }^{43}$, D. L. Harrison ${ }^{16,48}$, M. Hauser ${ }^{15}$, D. Hestroffer ${ }^{40}$, S. T. Hodgkin ${ }^{16}$, H. E. Huckle ${ }^{30}$, A. Hutton ${ }^{49}$, G. Jasniewicz ${ }^{50}$, S. Jordan ${ }^{15}$, M. Kontizas ${ }^{51}$, A. J. Korn ${ }^{52}$, A. C. Lanzafame ${ }^{53,54}$,

M. Manteiga ${ }^{55}$, A. Moitinho ${ }^{56}$, K. Muinonen ${ }^{57,58}$, J. Osinde ${ }^{59}$, E. Pancino ${ }^{25,60}$, T. Pauwels ${ }^{41}$, J.-M. Petit ${ }^{61}$, A. Recio-Blanco ${ }^{21}$, A. C. Robin ${ }^{61}$, C. Siopis ${ }^{23}$, M. Smith ${ }^{30}$, K. W. Smith ${ }^{14}$, A. Sozzetti ${ }^{31}$, W. Thuillot ${ }^{40}$, W. van Reeven $^{49}$, Y. Viala ${ }^{13}$, U. Abbas ${ }^{31}$, A. Abreu Aramburu ${ }^{62}$, S. Accart ${ }^{63}$, J. J. Aguado ${ }^{5}$, P. M. Allan ${ }^{36}$, W. Allasia ${ }^{64}$, G. Altavilla ${ }^{1}$, M. A. Álvarez ${ }^{55}$, J. Alves ${ }^{65}$, A. H. Andrei ${ }^{66,67,38}$, E. Anglada Varela ${ }^{59,35}$, E. Antiche ${ }^{6}$, T. Antoja ${ }^{10}$, S. Antón ${ }^{68,69}$, B. Arcay ${ }^{55}$, N. Bach ${ }^{49}$, S. G. Baker ${ }^{30}$, L. Balaguer-Núñez ${ }^{6}$, C. Barache ${ }^{38}$, C. Barata ${ }^{56}$, A. Barbier ${ }^{63}$,

F. Barblan ${ }^{2}$, D. Barrado y Navascués ${ }^{70}$, M. Barros ${ }^{56}$, M. A. Barstow ${ }^{71}$, U. Becciani ${ }^{54}$, M. Bellazzini ${ }^{1}$, A. Bello García $^{72}$, V. Belokurov ${ }^{16}$, P. Bendjoya ${ }^{21}$, A. Berihuete ${ }^{73}$, L. Bianchi ${ }^{64}$, O. Bienaymé ${ }^{47}$, F. Billebaud ${ }^{27}$,

N. Blagorodnova ${ }^{16}$, S. Blanco-Cuaresma ${ }^{2,27}$, T. Boch ${ }^{47}$, A. Bombrun ${ }^{44}$, R. Borrachero ${ }^{6}$, S. Bouquillon ${ }^{38}$, G. Bourda $^{27}$,

A. Bragaglia ${ }^{1}$, M. A. Breddels ${ }^{74}$, N. Brouillet ${ }^{27}$, T. Brüsemeister ${ }^{15}$, B. Bucciarelli ${ }^{31}$, P. Burgess $^{16}$, R. Burgon ${ }^{33}$, A. Burlacu ${ }^{22}$, D. Busonero ${ }^{31}$, R. Buzzi ${ }^{31}$, E. Caffau ${ }^{13}$, J. Cambras ${ }^{75}$, H. Campbell ${ }^{16}$, R. Cancelliere ${ }^{76}$,

T. Cantat-Gaudin ${ }^{12}$, T. Carlucci ${ }^{38}$, J. M. Carrasco ${ }^{6}$, M. Castellani ${ }^{77}$, P. Charlot ${ }^{27}$, J. Charnas ${ }^{7}$, A. Chiavassa ${ }^{21}$, M. Clotet ${ }^{6}$, G. Cocozza ${ }^{1}$, R. S. Collins ${ }^{43}$, G. Costigan ${ }^{11}$, F. Crifo ${ }^{13}$, N. J. G. Crosss ${ }^{43}$, M. Crosta ${ }^{31}$, C. Crowley ${ }^{44}$, C. Dafonte ${ }^{55}$, Y. Damerdji ${ }^{45,78}$, A. Dapergolas ${ }^{39}$, P. David ${ }^{40}$, M. David ${ }^{79}$, P. De Cat ${ }^{41}$, F. de Felice ${ }^{80}$, P. de Laverny ${ }^{21}$, F. De Luise ${ }^{81}$, R. De $\mathrm{March}^{34}$, R. de Souza ${ }^{82}$, J. Debosscher ${ }^{28}$, E. del Pozo ${ }^{49}$, M. Delbo ${ }^{21}$, A. Delgado ${ }^{16}$,

H. E. Delgado ${ }^{5}$, P. Di Matteo ${ }^{13}$, S. Diakite ${ }^{61}$, E. Distefano ${ }^{54}$, C. Dolding ${ }^{30}$, S. Dos Anjos ${ }^{82}$, P. Drazinos ${ }^{51}$, J. Durán ${ }^{59}$, Y. Dzigan ${ }^{83,84}$, B. Edvardsson ${ }^{52}$, H. Enke ${ }^{85}$, N. W. Evans ${ }^{16}$, G. Eynard Bontemps ${ }^{63}$, C. Fabre $^{86}$, M. Fabrizio ${ }^{60,81}$, A. J. Falcão ${ }^{87}$, M. Farràs Casas $^{6}$, L. Federici ${ }^{1}$, G. Fedorets ${ }^{57}$, J. Fernández-Hernández ${ }^{35}$, P. Fernique ${ }^{47}$, A. Fienga ${ }^{88}$, F. Figueras ${ }^{6}$, F. Filippi ${ }^{34}$, K. Findeisen ${ }^{13}$, A. Fonti ${ }^{34}$, M. Fouesneau ${ }^{14}$, E. Fraile ${ }^{89}$, M. Fraser ${ }^{16}$, J. Fuchs ${ }^{90}$, M. Gai ${ }^{31}$, S. Galleti ${ }^{1}$, L. Galluccio ${ }^{21}$, D. Garabato ${ }^{55}$, F. García-Sedano ${ }^{5}$, N. Garralda ${ }^{6}$, P. Gavras ${ }^{13,39,51}$, J. Gerssen ${ }^{85}$, R. Geyer ${ }^{18}$, G. Gilmore $^{16}$, S. Girona ${ }^{91}$, G. Giuffrida ${ }^{60}$, M. Gomes ${ }^{56}$, A. González-Marcos ${ }^{92}$, J. González-Núñez ${ }^{35,93}$,

J. J. González-Vidal ${ }^{6}$, M. Granvik ${ }^{57}$, A. Guerrier ${ }^{63}$, P. Guillout ${ }^{47}$, J. Guiraud ${ }^{22}$, A. Gúrpide ${ }^{6}$, R. Gutiérrez-Sánchez ${ }^{26}$, L. P. Guy ${ }^{7}$, R. Haigron ${ }^{13}$, D. Hatzidimitriou ${ }^{51,39}$, M. Haywood ${ }^{13}$, U. Heiter ${ }^{52}$, A. Helmi ${ }^{74}$, D. Hobbs ${ }^{20}$, W. Hofmann ${ }^{15}$,

B. Holl ${ }^{2}$, G. Holland ${ }^{16}$, J. A. S. Hunt ${ }^{30}$, A. Hypki ${ }^{11}$, V. Icardi ${ }^{34}$, M. Irwin ${ }^{16}$, G. Jevardat de Fombelle ${ }^{7}$, P. Jofré ${ }^{16,27}$,

P. G. Jonker ${ }^{94,29}$, A. Jorissen ${ }^{23}$, F. Julbe ${ }^{6}$, A. Karampelas ${ }^{51,39}$, A. Kochoska ${ }^{95}$, R. Kohley ${ }^{19}$, K. Kolenberg $96,28,97$,

E. Kontizas ${ }^{39}$, S. E. Koposov ${ }^{16}$, G. Kordopatis ${ }^{85,21}$, P. Koubsky ${ }^{90}$, A. Krone-Martins ${ }^{56}$, M. Kudryashova ${ }^{40}$,

R. K. Bachchan ${ }^{20}$, F. Lacoste-Seris ${ }^{63}$, A. F. Lanza ${ }^{54}$, J.-B. Lavigne ${ }^{63}$, C. Le Poncin-Lafitte ${ }^{38}$, Y. Lebreton ${ }^{13,98}$, T. Lebzelter ${ }^{65}$, S. Leccia ${ }^{3}$, N. Leclerc ${ }^{13}$, I. Lecoeur-Taibi ${ }^{7}$, V. Lemaitre ${ }^{63}$, H. Lenhardt ${ }^{15}$, F. Leroux ${ }^{63}$, S. Liao $^{31,99}$,

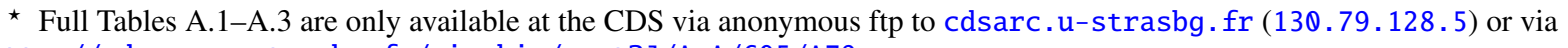
http://cdsarc.u-strasbg.fr/viz-bin/qcat?]/A+A/605/A79

$\star \star$ Corresponding author: G. Clementini,

e-mail: gisella.clementini@oabo.inaf.it 
E. Licata ${ }^{64}$, H. E. P. Lindstrøm ${ }^{32,100}$, T. A. Lister ${ }^{101}$, E. Livanou ${ }^{51}$, A. Lobel ${ }^{41}$, W. Löffler ${ }^{15}$, M. López ${ }^{70}$, D. Lorenz ${ }^{65}$, I. MacDonald ${ }^{43}$, T. Magalhães Fernandes ${ }^{87}$, S. Managau ${ }^{63}$, R. G. Mann ${ }^{43}$, G. Mantelet ${ }^{15}$, O. Marchal ${ }^{13}$,

J. M. Marchant ${ }^{102}$, S. Marinoni ${ }^{77,60}$, P. M. Marrese ${ }^{77,60}$, G. Marschalkó ${ }^{8,103}$, D. J. Marshall ${ }^{104}$, J. M. Martín-Fleitas ${ }^{49}$, M. Martino ${ }^{34}$, N. Mary ${ }^{63}$, G. Matijevič ${ }^{85}$, P. J. McMillan ${ }^{20}$, S. Messina ${ }^{54}$, D. Michalik ${ }^{20}$, N. R. Millar ${ }^{16}$,

B. M. H. Miranda ${ }^{56}$, D. Molina ${ }^{6}$, M. Molinaro ${ }^{105}$, L. Molnár ${ }^{8}$, M. Moniez ${ }^{106}$, P. Montegriffo ${ }^{1}$, R. Mor ${ }^{6}$, A. Mora ${ }^{49}$, R. Morbidelli $^{31}$, T. Morel ${ }^{45}$, S. Morgenthaler ${ }^{107}$, D. Morris ${ }^{43}$, A. F. Mulone ${ }^{34}$, J. Narbonne ${ }^{63}$, G. Nelemans ${ }^{29,28}$,

L. Nicastro ${ }^{108}$, L. Noval ${ }^{63}$, C. Ordénovic ${ }^{21}$, J. Ordieres-Meré ${ }^{109}$, P. Osborne ${ }^{16}$, C. Pagani ${ }^{71}$, I. Pagano ${ }^{54}$, F. Pailler ${ }^{22}$, H. Palacin ${ }^{63}$, L. Palaversa ${ }^{2}$, P. Parsons ${ }^{26}$, M. Pecoraro ${ }^{64}$, R. Pedrosa ${ }^{110}$, H. Pentikäinen ${ }^{57}$, B. Pichon ${ }^{21}$,

A. M. Piersimoni ${ }^{81}$, F.-X. Pineau ${ }^{47}$, E. Plachy ${ }^{8}$, G. Plum ${ }^{13}$, E. Poujoulet ${ }^{111}$, A. Prša ${ }^{112}$, L. Pulone ${ }^{77}$, S. Ragaini ${ }^{1}$,

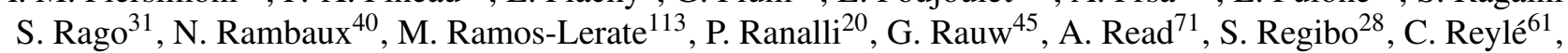
R. A. Ribeiro ${ }^{87}$, A. Riva ${ }^{31}$, G. Rixon ${ }^{16}$, M. Roelens ${ }^{2}$, M. Romero-Gómez ${ }^{6}$, N. Rowell ${ }^{43}$, F. Royer ${ }^{13}$, L. Ruiz-Dern ${ }^{13}$, G. Sadowski ${ }^{23}$, T. Sagristà Sellés ${ }^{15}$, J. Sahlmann ${ }^{19}$, J. Salgado ${ }^{59}$, E. Salguero ${ }^{59}$, M. Sarasso ${ }^{31}$, H. Savietto ${ }^{114}$,

M. Schultheis ${ }^{21}$, E. Sciacca ${ }^{54}$, M. Segol ${ }^{115}$, J.C. Segovia ${ }^{35}$, D. Segransan ${ }^{2}$, I-C. Shih ${ }^{13}$, R. Smareglia ${ }^{105}$, R. L. Smart ${ }^{31}$, E. Solano $^{70,116}$, F. Solitro ${ }^{34}$, R. Sordo ${ }^{12}$, S. Soria Nieto ${ }^{6}$, J. Souchay ${ }^{38}$, A. Spagna ${ }^{31}$, F. Spoto ${ }^{21}$, U. Stampa ${ }^{15}$, I. A. Steele ${ }^{102}$, H. Steidelmüller ${ }^{18}$, C. A. Stephenson ${ }^{26}$, H. Stoev ${ }^{117}$, F. F. Suess ${ }^{16}$, M. Süveges ${ }^{7}$, J. Surdej ${ }^{45}$,

E. Szegedi-Elek ${ }^{8}$, D. Tapiador ${ }^{118,119}$, F. Taris ${ }^{38}$, G. Tauran ${ }^{63}$, M. B. Taylor ${ }^{120}$, R. Teixeira ${ }^{82}$, D. Terrett ${ }^{36}$, B. Tingley ${ }^{121}$, S. C. Trager $^{74}$, C. Turon ${ }^{13}$, A. Ulla ${ }^{122}$, E. Utrilla ${ }^{49}$, G. Valentini ${ }^{81}$, A. van Elteren ${ }^{11}$, E. Van Hemelryck ${ }^{41}$, M. van

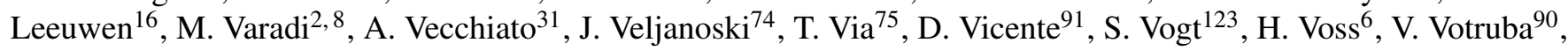
S. Voutsinas ${ }^{43}$, G. Walmsley ${ }^{22}$, M. Weiler ${ }^{6}$, K. Weingrill ${ }^{85}$, T. Wevers ${ }^{29}$, Ł. Wyrzykowski $^{16,124}$, A. Yoldas ${ }^{16}$, M. Žerjal ${ }^{95}$, S. Zucker ${ }^{83}$, C. Zurbach ${ }^{50}$, T. Zwitter ${ }^{95}$, A. Alecu ${ }^{16}$, M. Allen ${ }^{10}$, C. Allende Prieto ${ }^{30,125,126}$, A. Amorim ${ }^{56}$, G. Anglada-Escudé ${ }^{6}$, V. Arsenijevic ${ }^{56}$, S. Azaz ${ }^{10}$, P. Balm ${ }^{26}$, M. Beck ${ }^{7}$, H.-H. Bernstein ${ }^{\dagger 15}$, L. Bigot ${ }^{21}$, A. Bijaoui ${ }^{21}$, C. Blasco ${ }^{127}$, M. Bonfigli ${ }^{81}$, G. Bono ${ }^{77}$, S. Boudreault ${ }^{30,128}$, A. Bressan ${ }^{129}$, S. Brown ${ }^{16}$, P.-M. Brunet ${ }^{22}$, P. Bunclark ${ }^{\dagger 16}$, R. Buonanno ${ }^{77}$, A. G. Butkevich ${ }^{18}$, C. Carret ${ }^{110}$, C. Carrion ${ }^{5}$, L. Chemin ${ }^{27,}{ }^{130}$, F. Chéreau ${ }^{13}$, L. Corcione ${ }^{31}$, E. Darmigny ${ }^{22}$, K. S. de Boer ${ }^{131}$, P. de Teodoro ${ }^{35}$, P. T. de Zeeuw ${ }^{11,132}$, C. Delle Luche ${ }^{13,63}$, C. D. Domingues ${ }^{133}$, P. Dubath ${ }^{7}$, F. Fodor 22 , B. Frézouls 22 , A. Fries ${ }^{6}$, D. Fustes ${ }^{55}$, D. Fyfe ${ }^{71}$, E. Gallardo ${ }^{6}$, J. Gallegos ${ }^{35}$, D. Gardiol ${ }^{31}$, M. Gebran 6,134 , A. Gomboc ${ }^{95,135}$, A. Gómez ${ }^{13}$, E. Grux ${ }^{61}$, A. Gueguen ${ }^{13,136}$, A. Heyrovsky ${ }^{43}$, J. Hoar ${ }^{19}$, G. Iannicola ${ }^{77}$, Y. Isasi Parache ${ }^{6}$, A.-M. Janotto ${ }^{22}$, E. Joliet ${ }^{44}$, 137, A. Jonckheere ${ }^{41}$, R. Keil ${ }^{138,139}$, D.-W. Kim ${ }^{14}$, P. Klagyivik ${ }^{8}$, J. Klar ${ }^{85}$, J. Knude ${ }^{32}$, O. Kochukhov ${ }^{52}$, I. Kolka ${ }^{140}$, J. Kos ${ }^{95,141}$, A. Kutka ${ }^{90,142}$, V. Lainey ${ }^{40}$, D. LeBouquin ${ }^{63}$, C. Liu ${ }^{14,143}$, D. Loreggia ${ }^{31}$, V. V. Makarov ${ }^{144}$, M. G. Marseille ${ }^{63}$, C. Martayan ${ }^{41,145}$,

O. Martinez-Rubi ${ }^{6}$, B. Massart ${ }^{21,63,146}$, F. Meynadier ${ }^{13,38}$, S. Mignot ${ }^{13}$, U. Munari ${ }^{12}$, A.-T. Nguyen 22 , T. Nordlander ${ }^{52}$, K. S. O’Flaherty ${ }^{147}$, P. Ocvirk ${ }^{85,47}$, A. Olias Sanz ${ }^{148}$, P. Ortiz ${ }^{71}$, J. Osorio ${ }^{68}$, D. Oszkiewicz ${ }^{57,149}$, A. Ouzounis ${ }^{43}$, P. Park ${ }^{2}$, E. Pasquato ${ }^{23}$, C. Peltzer ${ }^{16}$, J. Peralta ${ }^{6}$, F. Péturaud ${ }^{13}$, T. Pieniluoma ${ }^{57}$, E. Pigozzi ${ }^{34}$, J. Poels ${ }^{\dagger 4}$, G. Prat ${ }^{150}$, T. Prod'homme ${ }^{11,151}$, F. Raison ${ }^{152,136}$, J. M. Rebordao ${ }^{133}$, D. Risquez ${ }^{11}$, B. Rocca-Volmerange ${ }^{153}$, S. Rosen ${ }^{30,71}$, M. I. Ruiz-Fuertes ${ }^{7}$, F. Russo ${ }^{31}$, I. Serraller Vizcaino ${ }^{154}$, A. Short ${ }^{10}$, A. Siebert ${ }^{47,85}$, H. Silva ${ }^{87}$, D. Sinachopoulos ${ }^{39}$, E. Slezak ${ }^{21}$, M. Soffel ${ }^{18}$, D. Sosnowska ${ }^{2}$, V. Straižys ${ }^{155}$, M. ter Linden ${ }^{44,156}$, D. Terrell ${ }^{157}$, S. Theil ${ }^{158}$, C. Tiede ${ }^{14,159}$,

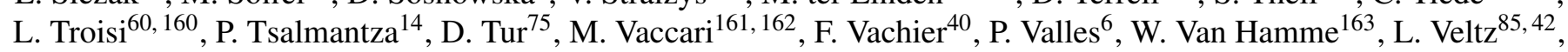
J. Virtanen ${ }^{57,58}$, J.-M. Wallut ${ }^{22}$, R. Wichmann ${ }^{164}$, M. I. Wilkinson ${ }^{16,71}$, H. Ziaeepour ${ }^{61}$, and S. Zschocke ${ }^{18}$

(Affiliations can be found after the references)

Received 18 October 2016 / Accepted 26 April 2017

\section{ABSTRACT}

Context. Parallaxes for 331 classical Cepheids, 31 Type II Cepheids, and 364 RR Lyrae stars in common between Gaia and the HIPPARCOS and Tycho-2 catalogues are published in Gaia Data Release 1 (DR1) as part of the Tycho-Gaia Astrometric Solution (TGAS).

Aims. In order to test these first parallax measurements of the primary standard candles of the cosmological distance ladder, which involve astrometry collected by Gaia during the initial 14 months of science operation, we compared them with literature estimates and derived new period-luminosity $(P L)$, period-Wesenheit $(P W)$ relations for classical and Type II Cepheids and infrared $P L, P L$-metallicity $(P L Z)$, and optical luminosity-metallicity $\left(M_{V}-[\mathrm{Fe} / \mathrm{H}]\right)$ relations for the RR Lyrae stars, with zero points based on TGAS.

Methods. Classical Cepheids were carefully selected in order to discard known or suspected binary systems. The final sample comprises 102 fundamental mode pulsators with periods ranging from 1.68 to 51.66 days (of which 33 with $\sigma_{\varpi} / \varpi<0.5$ ). The Type II Cepheids include a total of $26 \mathrm{~W}$ Virginis and BL Herculis stars spanning the period range from 1.16 to 30.00 days (of which only 7 with $\sigma_{\varpi} / \varpi<0.5$ ). The RR Lyrae stars include 200 sources with pulsation period ranging from 0.27 to 0.80 days (of which 112 with $\sigma_{\varpi} / \varpi<0.5$ ). The new relations were computed using multi-band $\left(V, I, J, K_{\mathrm{s}}\right)$ photometry and spectroscopic metal abundances available in the literature, and by applying three alternative approaches: (i) linear least-squares fitting of the absolute magnitudes inferred from direct transformation of the TGAS parallaxes; (ii) adopting astrometrybased luminosities; and (iii) using a Bayesian fitting approach. The last two methods work in parallax space where parallaxes are used directly, thus maintaining symmetrical errors and allowing negative parallaxes to be used. The TGAS-based $P L, P W, P L Z$, and $M_{V}-[\mathrm{Fe} / \mathrm{H}]$ relations are discussed by comparing the distance to the Large Magellanic Cloud provided by different types of pulsating stars and alternative fitting methods. 
Results. Good agreement is found from direct comparison of the parallaxes of RR Lyrae stars for which both TGAS and HST measurements are available. Similarly, very good agreement is found between the TGAS values and the parallaxes inferred from the absolute magnitudes of Cepheids and RR Lyrae stars analysed with the Baade-Wesselink method. TGAS values also compare favourably with the parallaxes inferred by theoretical model fitting of the multi-band light curves for two of the three classical Cepheids and one RR Lyrae star, which were analysed with this technique in our samples. The $K$-band $P L$ relations show the significant improvement of the TGAS parallaxes for Cepheids and RR Lyrae stars with respect to the HIPPARCos measurements. This is particularly true for the RR Lyrae stars for which improvement in quality and statistics is impressive. Conclusions. TGAS parallaxes bring a significant added value to the previous HIPPARcos estimates. The relations presented in this paper represent the first Gaia-calibrated relations and form a work-in-progress milestone report in the wait for Gaia-only parallaxes of which a first solution will become available with Gaia Data Release 2 (DR2) in 2018.

Key words astrometry - parallaxes - stars: distances - stars: variables: Cepheids - stars: variables: RR Lyrae - methods: data analysis

\section{Introduction}

On 14 September 2016, photometry and astrometry data collected by the Gaia mission during the first 14 months of science operation were released to the public with the Gaia first data release (hereafter Gaia DR1; Gaia Collaboration 2016b,a). In particular, the Gaia DR1 catalogue includes positions, proper motions, and parallaxes for about 2 million stars in common between Gaia and the HIPPARCos and Tycho-2 catalogues computed as part of the Tycho-Gaia Astrometric Solution (TGAS); the principles of TGAS are discussed in Michalik et al. (2015) and the results published in Gaia DR1 are described in detail in Lindegren et al. (2016). Among the TGAS sources is a sample of Galactic pulsating stars which includes 331 classical Cepheids, 31 Type II Cepheids, and 364 RR Lyrae stars. As part of a number of checks performed within the Gaia Data Processing and Analysis Consortium (DPAC), we have tested TGAS parallaxes for Cepheids and RR Lyrae stars by building canonical relations followed by these variable stars, such as the period-luminosity $(P L)$ and period-Wesenheit $(P W)$ relations for classical and Type II Cepheids and the infrared $P L$, $P L$-metallicity $(P L Z)$, and optical luminosity-metallicity $\left(M_{V^{-}}\right.$ $[\mathrm{Fe} / \mathrm{H}])$ relations for RR Lyrae stars, with zero points based on TGAS parallaxes. The results of these tests are presented in this paper.

Thanks to the characteristic $P L$ relation discovered at the beginning of the last century by Mrs Henrietta Swan Leavitt (1868-1921), classical Cepheids have become the basis of an absolute calibration of the extragalactic distance scale (see e.g. Freedman et al. 2001; Saha et al. 2006; Fiorentino et al. 2013; Riess et al. 2011, 2016, and references therein). The $P L$ is a statistical relation with an intrinsic dispersion caused by the finite width of the instability strip for pulsating stars. This dispersion is particularly significant in the optical bands (e.g. $B, V$ ), where it is of the order of $\pm 0.25 \mathrm{mag}$, but decreases moving towards longer wavelengths becoming less than $\sim \pm 0.1 \mathrm{mag}$ in the near- and mid-infrared (NIR and MIR) filters (see e.g. Madore \& Freedman 1991; Caputo et al. 2000a; Marconi et al. 2005; Ngeow et al. 2012; Ripepi et al. 2012; Inno et al. 2013; Gieren et al. 2013, and references therein). Main open issues concerning the use of the Cepheid $P L$ for extragalactic distance determinations are the dependence of the $P L$ relation on chemical composition, on which no general consensus has yet been reached in the literature, and the possible non-linearity of the Cepheid $P L$ relations at the longest periods, for which some authors find evidence in the form of a break around 10 days, with a clear corresponding change in the $P L$ slope in $B, V, R$, and $I$ (see e.g. Ngeow \& Kanbur 2006; Tammann et al. 2003). The metallicity (and helium) dependence and the non-linearity effect, as well as the effect of the finite intrinsic width of the instability strip mentioned above, all decrease when moving from optical to NIR and MIR passbands (see e.g. Madore \& Freedman 1991; Caputo et al. 2000a; Marconi et al. 2005; Ripepi et al. 2012, 2016; Inno et al. 2013; Gieren et al. 2013, and references therein).

When optical bands are used great advantages are obtained by adopting reddening-free formulations of the $P L$ relation, called Wesenheit functions $(P W s)$ (see Madore 1982; Caputo et al. 2000a; Ripepi et al. 2012). These relations include a colour term, thus partially correcting for the intrinsic width of the instability strip, whose coefficient is given by the ratio of total to selective extinction. The Wesenheit relation in the $V, I$ bands, $P W(V, I)$, is often adopted to derive accurate extragalactic distances as it is widely recognised to be little dependent on metallicity (see e.g. Bono et al. 2010, and references therein). Other filter combinations extending to the NIR are also commonly used in the literature (see e.g. Riess et al. 2011, 2016; Fiorentino et al. 2013; Ripepi et al. 2012, 2016). However, all these relations need an accurate calibration of their zero points and a quantitative assessment of the dependence of slope and zero point on the chemical composition as any systematic effects on the coefficients of both $P L$ and $P W$ relations directly propagates in the calibration of the secondary distance indicators and the estimate of the Hubble constant, $H_{0}$. Gaia will play a crucial role in definitely addressing all these issues of the Cepheid-based distance ladder.

On the other hand, an alternative and independent route to $H_{0}$ using the cosmic "distance ladder" method is provided by Population II pulsating stars such as the RR Lyrae stars (see e.g. Beaton et al. 2016, and references therein); the Type II Cepheids; and the SX Phoenicis variables, which are old $(t \gtrsim$ $10 \mathrm{Gyr}$ ), subsolar mass variables, that typically populate globular clusters and galactic halos. While Type II Cepheids and SX Phoenicis stars follow PL relations, the standard candle commonly associated with RR Lyrae stars is the relation existing between the mean absolute visual magnitude $\left\langle M_{V}(R R)\right\rangle$ and the iron content $[\mathrm{Fe} / \mathrm{H}]$, usually assumed in a linear form: $M_{V}(R R)=a[\mathrm{Fe} / \mathrm{H}]+b$. Current determinations of the slope $a$ and zero point $b$ of this relation span a wide range of values (see e.g. Clementini et al. 2003; Cacciari \& Clementini 2003; Marconi 2015, and references therein) and theoretical investigations based on evolutionary and pulsation models also suggest a change in the slope at $[\mathrm{Fe} / \mathrm{H}] \approx-1.5 \mathrm{dex}$ (see e.g. Caputo et al. 2000b; Cassisi et al. 1998; Lee et al. 1990). The other characteristic relation that makes RR Lyrae stars fundamental primary distance indicators for systems mainly composed of Population II stars is the $P L$ relation they conform to at infrared wavelengths and in the $K$ band $(2.2 \mu \mathrm{m})$ in particular, as first pointed out in the pioneering investigations of Longmore et al. (1986, 1990). Owing to the strict relation between the $V-K$ colour 
and the effective temperature, and between the latter quantity and the pulsation period, the nearly horizontal distribution of the RR Lyrae stars in the $M_{V}$ versus $\log P$ plane evolves into a strict $P L$ relation in the $M_{K}$ versus $\log P$ plane (see e.g. Fig. 2 in Catelan et al. 2004), according to which longer periods correspond to brighter pulsators in the $K$ band. It has also been demonstrated (Bono et al. 2001) that the intrinsic dispersion of the $P L(K)$ relation drastically decreases when metallicity differences and evolutionary effects are taken into account. However, coefficients and the zero point of the $M_{K}-\log P-[\mathrm{Fe} / \mathrm{H}] \mathrm{re}-$ lation (hereafter $P M_{K} Z$ ) are still a matter of debate in the literature and may differ significantly from one study to another (see e.g. Marconi 2015). Bono et al. (2003) and Catelan et al. (2004) analysed the $P M_{K} Z$ from the semi-theoretical and theoretical point of view and found a non-negligible dependence of the RR Lyrae $K$-band absolute magnitude, $M_{K}$, on metallicity: $b=0.231 \pm 0.012$ and $b=0.175$, respectively. Conversely, the dependence of the $K$-band luminosity on metallicity derived in empirical studies is generally much shallower (Del Principe et al. 2006) or even negligible (Sollima et al. 2006, 2008; Borissova et al. 2009; Muraveva et al. 2015). The values in the literature for the dependence of $M_{K}$ on period vary from -2.101 (Bono et al. 2003) to -2.73 (Muraveva et al. 2015).

In this paper we use TGAS parallaxes of local Cepheids and RR Lyrae stars along with literature $V, I, J, K_{\mathrm{s}}, W_{1}$ photometry to compute new $P L, P W$, and $M_{V}-[\mathrm{Fe} / \mathrm{H}]$ relations through a variety of methods and compare their results. This enables us to test TGAS parallaxes for these primary standard candles. Estimation of distances from trigonometric parallaxes is not straightforward and is still a matter of debate. The direct transformation to distance (and then absolute magnitude) by parallax inversion is not often advisable if errors are large since it causes asymmetric errors in the magnitudes and does not allow the use of negative parallaxes. Methods that operate in parallax space such as the Astrometry-Based Luminosity (ABL, Arenou \& Luri 1999) and Bayesian approaches are to be preferred. In this paper we adopt the least-squares fit of the absolute magnitudes obtained from direct transformation of the parallaxes, the ABL method, and a Bayesian approach to fit the various relations that Cepheids and RR Lyrae stars conform to, then compare the results that different types of variables and different fitting methods provide for the distance to the Large Magellanic Cloud (LMC). Far from seeking results on the cosmic distance ladder as re-designed by these first Gaia measurements, the exercise presented in this paper is meant to assess the limitations and potential of this first astrometry solution and to compare different methods of handling parallaxes. The present approach partially differs from the photometric parallax approach adopted in Lindegren et al. (2016) and Casertano et al. (2017), where literature Cepheid PL relations (whether in the visual or the NIR) are assumed to probe TGAS parallaxes of classical Cepheids; there is hope that our approach is less prone to shortcomings arising from the intrinsic width of the Cepheid instability strip and the poor knowledge of the universality, linearity, and metallicity-dependence of the reference relations used in the above-mentioned studies.

The paper is organised as follows. In Sect. 2 we present the samples of Cepheids (both classical and Type II) and RR Lyrae stars we have analysed, describe how they were selected, and compare their TGAS parallaxes with parallax values (HIPPARCOS and/or HST) available in the literature for some of them, with the parallaxes inferred from the theoretical modelling of the light curves and from Baade-Wesselink studies. In Sect. 3 we analyse possible biases that affect the Cepheid and RR Lyrae samples and describe the methods we used to fit the various relations of these variable stars. In Sect. 4 we present the photometric dataset used for the classical Cepheids and the derivation of the corresponding $P L$ and $P W$ relations. Section 5 is devoted to the Type II Cepheids, and Sect. 6 to the RR Lyrae stars. In Sect. 7 we discuss the TGAS-based relations derived in the previous sections by comparing the distance to the Large Magellanic Cloud they provided and present a few concluding remarks.

\section{Cepheid and RR Lyrae samples}

\subsection{Sample selection}

The magnitude distribution of the sources for which a parallax measurement is available in Gaia DR1 is shown in Fig. 1 in Gaia Collaboration (2016a) and includes sources with a mean $G$-band apparent magnitude between $\sim 5$ and $\sim 13.5$ mag (but only very few with $G \lesssim 7 \mathrm{mag}$ ). The typical uncertainty of the TGAS parallaxes is 0.3 milliarcsecond (mas), to which a systematic component of 0.3 mas should be added. This systematic component arises from model assumptions and simplifications of data processing for DR1, among which, mainly, position and colour of the sources, as widely discussed in Lindegren et al. (2016) and also summarised in Sect. 6 of Gaia Collaboration (2016a). Since TGAS parallaxes are available for sources observed by Tycho-2 (Høg et al. 2000) - only a fraction of which are also in the HIPPARCos catalogue (ESA 1997; van Leeuwen 2007a) - in order to build the largest possible samples we used the list of Cepheids and RR Lyrae stars in the Tycho-2 catalogue as reference. To create this list we cross-identified the Tycho-2 whole catalogue with the General Catalog of Variable Stars (GCVS database; Samus et al. 2007-2015), which contains a total of 1100 between classical and Type II Cepheids, and with the David Dunlap Observatory Database of Galactic Classical Cepheids (DDO ${ }^{1}$; Fernie et al. 1995), which contains over 500 classical Cepheids. In particular, according to the variability types in the GCVS, in these selections we included, under the definition of Classical Cepheids, the following types: Cepheids and classical Cepheids or Delta Cephei-type variables (CEP, CEP(B), DCEP, DCEPS, and DCEPS(B), as labelled in the GCVS). Then we included under Type II Cepheids, the following types: CW, CWA, CWB, RV, RVA and RVB. Crossmatching these databases with the Tycho-2 general catalogue $(\gtrsim 2.5$ billion sources) and following supplements ( $~ 18$ thousand sources) we found final samples of 388 classical and 33 Type II Cepheids ${ }^{2}$. We then queried the tgas_source table in the Gaia Archive Core Systems (GACS) ${ }^{3}$ to retrieve TGAS parallaxes and Gaia $G$-band apparent magnitudes for the samples of 388 classical and 33 Type II Cepheids. Only for 331 of the classical Cepheids in our list are TGAS parallaxes and Gaia $G$ magnitudes actually available in GACS. They span $G$-band apparent magnitudes in the range $4.68 \leq G \leq 12.54 \mathrm{mag}$. Their parallaxes range from -1.610 to 6.214 mas, with parallax errors in the range from 0.215 to 0.958 mas, and with 29 sources having TGAS negative parallax. The error distribution of TGAS

\footnotetext{
1 http://wWw . astro.utoronto.ca/DD0/research/cepheids/ 2 The cross-match between Tycho-2 and GCVS sources was done using equatorial J2000 RA, Dec coordinates and assuming an astrometric error of 1 arcsec between catalogues. Conversely, we converted the DDO database equatorial B1950 coordinates to J2000 before matching the Tycho-2 and DDO catalogues and assumed 1-5 arcsec as maximum difference of the two sets of coordinates.

3 http://archives.esac.esa.int/gaia
} 


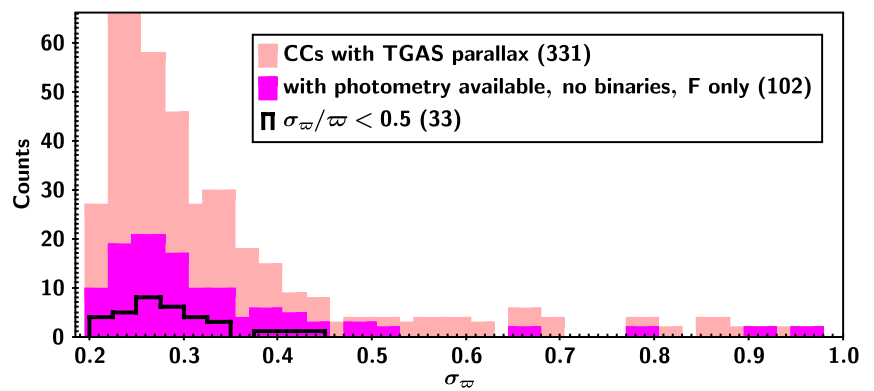

Fig. 1. Error distribution of TGAS parallaxes for classical Cepheids (CCs, in the label): whole sample (331 stars, pink), subsample with literature photometry after removing binaries and retaining only fundamental-mode $(\mathrm{F})$ pulsators (102 stars, magenta), subsample of the previous 102 sources retaining only stars with positive parallax and parallax errors $\sigma_{\varpi} / \varpi<0.5$ (33 stars, black contour). The bin size is 0.025 mas.

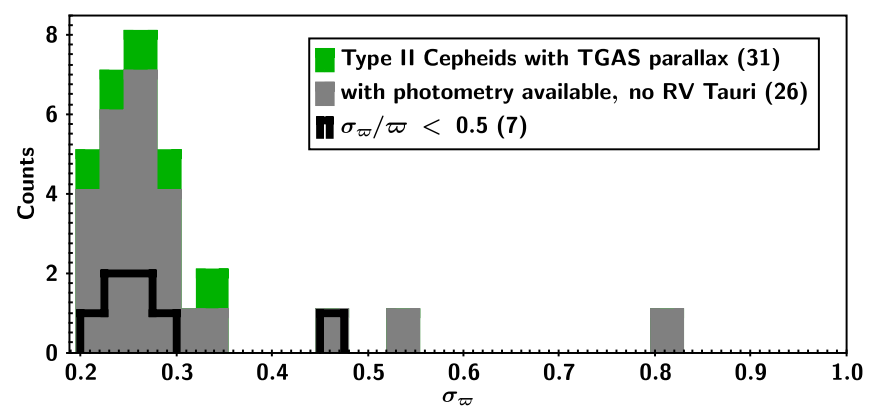

Fig. 2. Error distribution of TGAS parallaxes for Type II Cepheids: whole sample (31 stars, green), subsample with literature photometry and removing variables of RV Tauri type (26 stars, grey), subsample of the previous 26 sources retaining only stars with positive parallax and parallax errors $\sigma_{\varpi} / \varpi<0.5$ (7 stars, black contour). The bin size is 0.025 mas.

parallaxes for the 331 classical Cepheids is shown by the pink histogram in Fig. 1. Of the 33 Type II Cepheids, only 31 have $G$ magnitudes and TGAS parallaxes available. They span $G$-band apparent magnitudes in the range $6.89 \leq G \leq 12.10 \mathrm{mag}$. Their parallaxes range from -0.234 to 3.847 mas, with parallax errors from 0.219 mas to 0.808 mas, and with negative parallax for five of them. The error distribution of the TGAS parallax for the 31 Type II Cepheids is shown by the green histogram in Fig. 2.

Concerning the RR Lyrae stars, the GCVS (Samus et al. 2007-2015) contains information on 7954 such variables which are labelled as RR, RR(B), RR:, RRA, RRAB, RRAB:, RRC, RRC:, where ":" means uncertain classification. We crossmatched the GCVS RR Lyrae star sample against the Tycho-2 general catalogue and its supplements, and found 421 sources in common. Three sources, (S Eri, V2121 Cyg, and NZ Peg) have uncertain classification according to "The SIMBAD astronomical database" (Wenger et al. 2000) and were removed. We then cross-matched the remaining 418 sources against the tgas_source table in GACS and found a TGAS parallax for 364 of them. Values of the $G$-band apparent magnitude for these $364 \mathrm{RR}$ Lyrae stars are in the range $7.03 \leq G \leq 13.56 \mathrm{mag}$. Their parallaxes are in the range from -0.837 to 13.131 mas with parallax errors ranging from 0.209 to 0.967 mas; six stars have negative parallaxes. The error distribution of TGAS parallax for the 364 RR Lyrae stars is shown by the cyan histogram in Fig. 3.

Finally, the distribution on the sky of the 331 classical Cepheids, 31 Type II Cepheids, and 364 RR Lyrae stars considered in this paper is shown in Fig. 4; the red filled circles

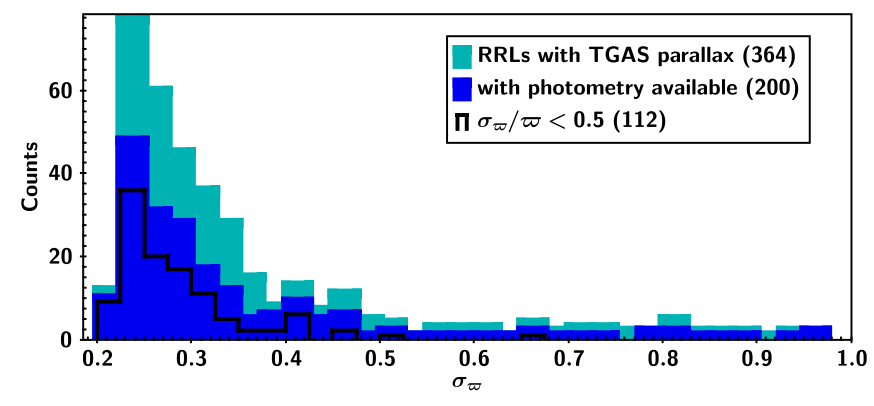

Fig. 3. Error distribution of the TGAS parallaxes for RR Lyrae stars (RRLs): whole sample (364 stars, cyan), subsample with literature photometry (200 stars, blue), subsample of the previous 200 sources retaining only stars with positive parallax and parallax errors $\sigma_{\varpi} / \varpi<0.5$ (112 stars, black contour). The bin size is 0.025 mas.

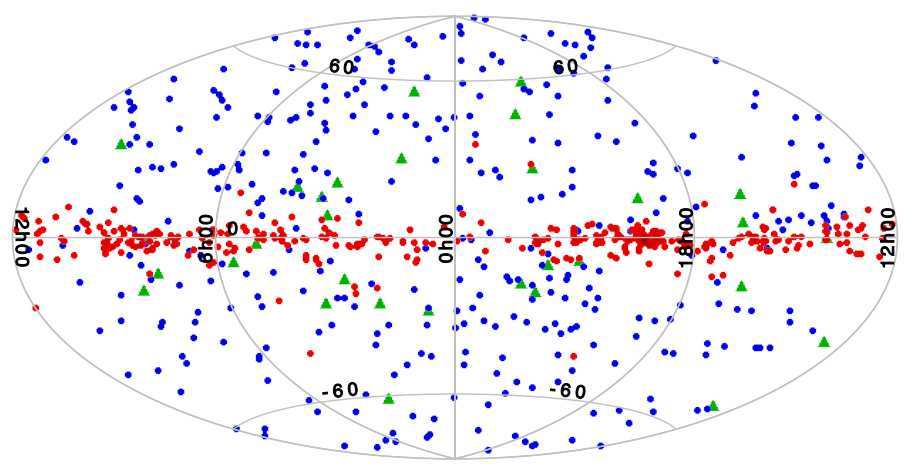

Fig. 4. Sky distribution, in Galactic coordinates, of the 331 classical Cepheids (red filled circles), 31 Type II Cepheids (green filled triangles), and 364 RR Lyrae stars (blue filled circles) discussed in this paper.

mark the classical Cepheids that, as expected, mainly concentrate in the Milky Way (MW) disc, and the green filled triangles and blue filled circles mark the Type II Cepheids and RR Lyrae stars, respectively, that nicely outline the MW halo. We note that by combining the results from these three different standard candles and the improved census of such variables that Gaia is expected to provide, it will be possible to further probe the MW 3D structure and the entire sky extension of the Galactic halo, a topic for which Gaia has already demonstrated its potential through the discovery of over 300 new RR Lyrae stars in the as yet unexplored far outskirts of one of our closest neighbours, the LMC (Clementini et al. 2016).

\subsection{Comparison with other parallax measurements}

Parallaxes obtained with the TGAS for classical and Type II Cepheids and for the RR Lyrae stars published in Gaia DR1 are listed in Tables A.1-A.3, where we also provide $G$-band magnitudes and other relevant photometric and spectroscopic information for these stars. In order to assess qualitatively the goodness of the TGAS parallaxes for Cepheids and RR Lyrae stars we compared the parallax values for variables having both TGAS and HIPPARCos measurements (248 classical Cepheids, 31 Type II Cepheids, and 188 RR Lyrae stars). The comparison of TGAS versus HIPPARCOS for the classical Cepheids is shown in Fig. 5 using black filled circles to mark the whole sample. We have labelled in the figure two stars, RW Cam and SY Nor, for which a significant discrepancy exists between HIPPARCos and TGAS parallax values. Both stars are known to have very bright close-by companions (Evans 1994; Fernie 2000). We also do not plot the three sources with the largest differences, namely V1477 


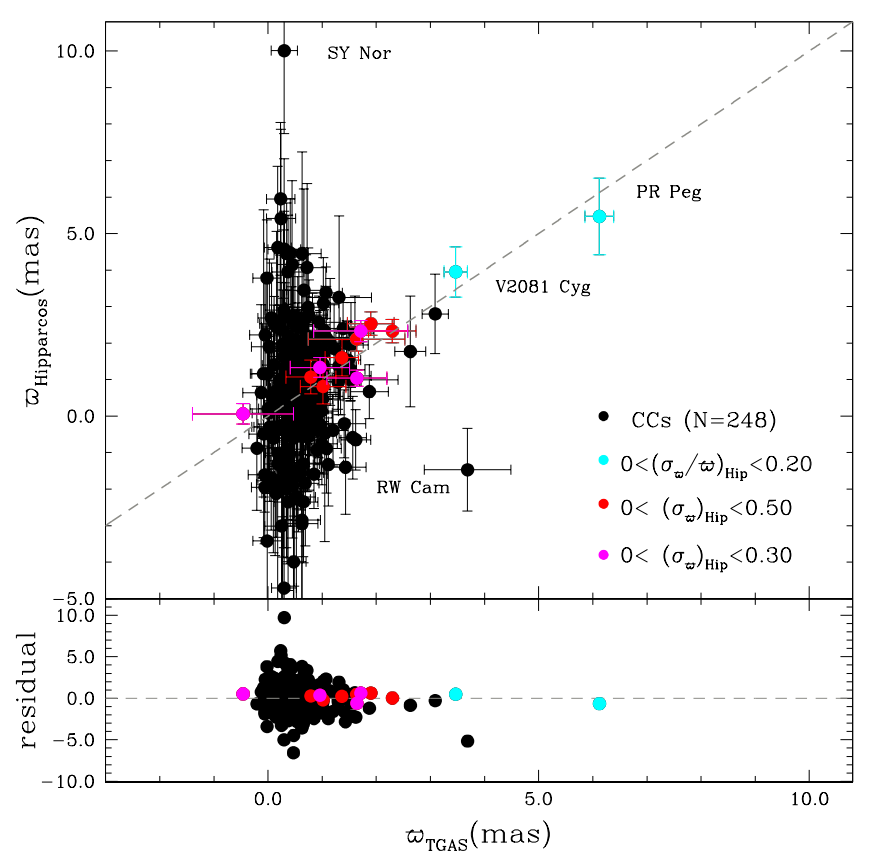

Fig. 5. Comparison between HIPPARCos and TGAS parallaxes obtained from a sample of 248 classical Cepheids which have both measurements. Red and magenta filled circles represent stars with $\left(\sigma_{\varpi}\right)_{\text {HIPPARCOS }}<0.50$ and 0.30 mas, respectively; cyan filled circles are two stars with $\left(\sigma_{\varpi} / \varpi\right)_{\text {HIPPARCOS }}<0.20$, namely V2081 Cyg and PR Peg. A dashed line shows the bisector. Residuals are TGAS HIPPARCos parallax values.

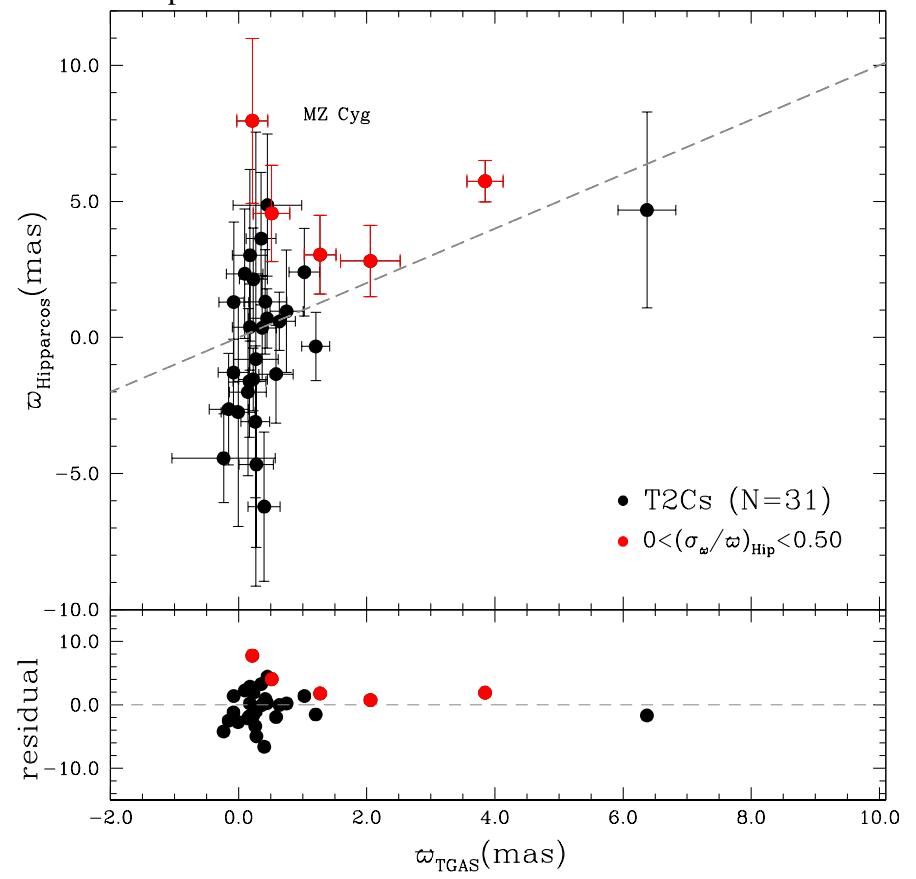

Fig. 6. Comparison between HIPPARCos and TGAS parallaxes obtained from the sample of 31 Type II Cepheids (T2Cs) which have both measurements. Red filled circles represent stars with $\left(\sigma_{\varpi} / \varpi\right)_{\text {HIPPARCOS }}<$ 0.50. A dashed line shows the bisector. Residuals are TGAS HIPPARCos parallax values.

Aql, UX Per, and AQ Pup. The TGAS-HIPPARcos comparison for classical Cepheids shows comforting results; the number of negative parallaxes has reduced from $32 \%$ in HIPPARCos to only 4\% in TGAS: of the 248 classical Cepheids, 79 have a negative HIPPARCos parallax compared with only 5 of them still

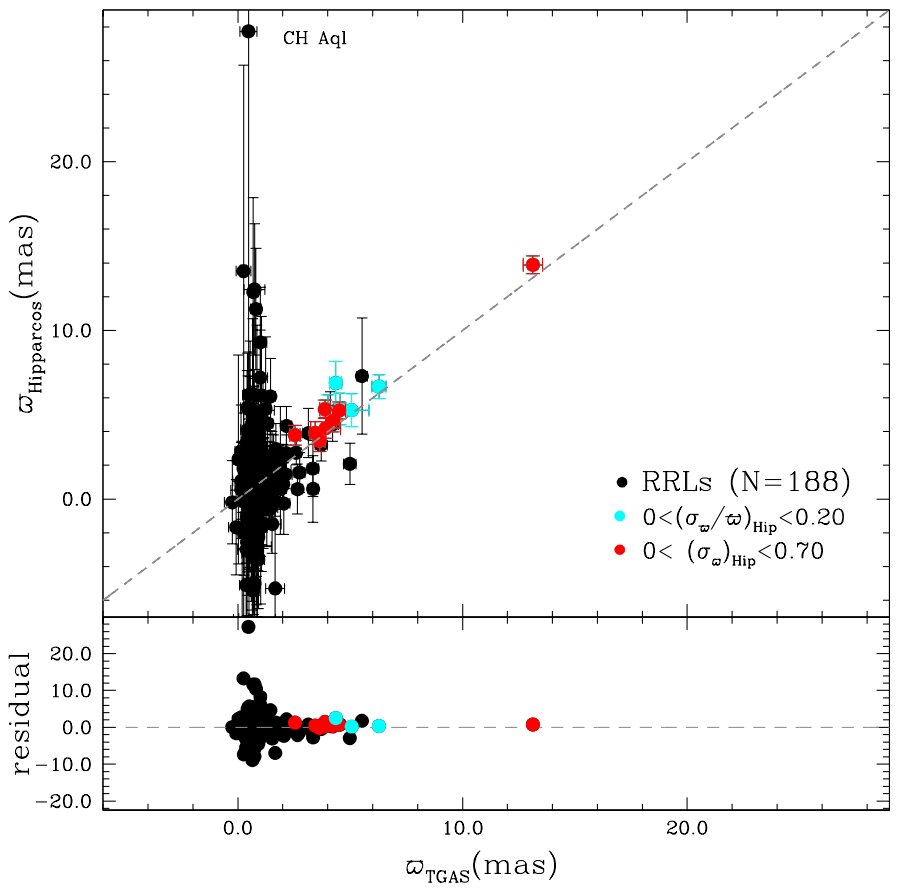

Fig. 7. Comparison between HIPPARcos and TGAS parallaxes obtained from a sample of 188 RR Lyrae stars (black filled circles) which have both measurements. Cyan filled circles mark sources with $\left(\sigma_{\varpi} / \varpi\right)_{\text {HIPPARCOS }}<0.20$. Red filled circles are RR Lyrae stars with $\left(\sigma_{\varpi}\right)_{\text {HIPPARCOS }}<0.70$. A dashed line shows the bisector. Residuals are TGAS - HIPPARCOS parallax values.

having negative parallax and an additional 6 stars for a total of 11 sources in TGAS. This is not surprising, since the fraction of negative parallaxes is expected to decrease when uncertainties get smaller. We have created different subsamples based on absolute and relative errors of the HIPPARCos parallaxes in order to highlight the samples with the most reliable parallaxes. Classical Cepheids with $\left(\sigma_{\varpi} / \varpi\right)_{\text {HIPPARCOS }}<0.20$ are marked in Fig. 5 by cyan filled circles; they are V2081 Cyg and PR Peg ${ }^{4}$. Red and magenta filled circles highlight stars with $\left(\sigma_{\varpi}\right)_{\text {HIPPARCOS }}<0.50$ and 0.30 mas, respectively. Increasing agreement between the TGAS and HIPPARCos results is found if we consider only sources with precise HIPPARCOS values, suggesting that more precise HIPPARCOS measures correspond to more precise TGAS measures. Figures 6 and 7 show the same test, but for Type II Cepheids and RR Lyrae stars, respectively. Red filled circles in Fig. 6 indicate Type II Cepheids with $\left(\sigma_{\varpi}\right)_{\text {HIPPARCOS }}<0.50$. Of the 31 Type II Cepheids with both HIPPARCos and TGAS parallaxes, 13 had a negative HIPPARCos parallax (42\% of the sample) compared with only 4 of them still having negative parallax and an additional 1 for a total of 5 sources (16\%) in TGAS. MZ Cyg is the source with the largest discrepancy between HIPPARCOS and TGAS among the Type II Cepheids with a positive parallax value. Red filled circles in Fig. 7 are RR Lyrae stars with $\left(\sigma_{\varpi}\right)_{\text {HIPPARCOS }}<0.70$, while cyan filled circles are the few RR Lyrae stars with $\left(\sigma_{\varpi} / \varpi\right)_{\text {HIPPARCos }}<0.20$. Of the 188 RR Lyrae stars with both HIPPARCos and TGAS parallax, 59 had a negative HIPPARCos parallax (31\% of the sample) compared with only 2 of them still having a negative parallax

4 Based on the periods and absolute magnitudes of these two stars, it was suggested to us that they might not be classical Cepheids; however, we double-checked the literature and found that both stars are still classified as Cepheids in the latest version of the General catalogue of variable stars: Version GCVS 5.1 (Samus et al. 2017). 
Table 1. Comparison between HIPPARCos, TGAS, and HST parallaxes.

\begin{tabular}{|c|c|c|c|c|c|c|c|c|}
\hline Name & ID $_{\text {HIPPARCOS }}{ }^{*}$ & $\begin{array}{c}\varpi_{\text {HIPPARCOS }} \\
\text { (mas) }\end{array}$ & $\begin{array}{c}\sigma \varpi_{\text {HIPPARCOS }} \\
\text { (mas) }\end{array}$ & $\begin{array}{c}\varpi_{\mathrm{TGAS}} \\
\text { (mas) }\end{array}$ & $\begin{array}{c}\sigma \varpi_{\mathrm{TGAS}} \\
(\mathrm{mas})\end{array}$ & $\begin{array}{l}\varpi_{\mathrm{HST}} \\
(\mathrm{mas})\end{array}$ & $\begin{array}{c}\sigma \varpi_{\mathrm{HST}} \\
(\mathrm{mas})\end{array}$ & HST Reference \\
\hline \multicolumn{9}{|c|}{ Classical Cepheids } \\
\hline $\mathrm{FF} \mathrm{Aql}{ }^{* *}$ & 93124 & 2.110 & \pm 0.330 & 1.640 & \pm 0.89 & 2.810 & \pm 0.180 & Benedict et al. (2007) \\
\hline SS CMa & 36088 & 0.400 & \pm 1.780 & 0.686 & \pm 0.234 & 0.348 & \pm 0.038 & Casertano et al. (2016) \\
\hline SY Aur & 24281 & -1.840 & \pm 1.720 & 0.687 & \pm 0.255 & 0.428 & \pm 0.054 & Riess et al. (2014) \\
\hline \multicolumn{9}{|c|}{ Type II Cepheids } \\
\hline VY Pyx & 434736 & 5.00 & \pm 0.44 & 3.85 & \pm 0.28 & 6.44 & \pm 0.23 & Benedict et al. (2011) \\
\hline \multicolumn{9}{|c|}{ RR Lyrae stars } \\
\hline RR Lyr & 95497 & 3.46 & \pm 0.64 & 3.64 & \pm 0.23 & 3.77 & \pm 0.13 & Benedict et al. (2011) \\
\hline RZ Cep & 111839 & 0.59 & \pm 1.48 & 2.65 & \pm 0.24 & $2.12(2.54)^{* * *}$ & \pm 0.16 & Benedict et al. (2011) \\
\hline SU Dra & 56734 & 0.20 & \pm 1.13 & 1.43 & \pm 0.29 & 1.42 & \pm 0.16 & Benedict et al. (2011) \\
\hline UV Oct & 80990 & 2.44 & \pm 0.81 & 2.02 & \pm 0.22 & 1.71 & \pm 0.10 & Benedict et al. (2011) \\
\hline XZ Cyg & 96112 & 2.29 & \pm 0.84 & 1.56 & \pm 0.23 & 1.67 & \pm 0.17 & Benedict et al. (2011) \\
\hline
\end{tabular}

Notes. ${ }^{(*)}$ van Leeuwen (2007b). ${ }^{(*)}$ Gallenne et al. (2012) have estimated the distance to FF Aql via the interferometric Baade-Wesselink technique; the corresponding parallax is $2.755 \pm 0.554$ mas. ${ }^{(* *)}$ Two different parallax values are provided for this star by Benedict et al. (2011); in the table we list both values.

(1\%) in TGAS. CH Aql is the source with the largest discrepancy between HIPPARCOS and TGAS among the RR Lyrae stars with positive parallax values. From these first global comparisons the improvement of Gaia with respect to HIPPARCos is straighforward and is even more so for the Population II standard candles, that is for RR Lyrae stars and Type II Cepheids.

Considering now the most accurate astrometric parallaxes available in the literature, we note that three classical Cepheids in our sample - FF Aquilae (FF Aql), SY Aurigae (SY Aur), and SS Canis Majoris (SS CMa) - have their parallax measured with the Hubble Space Telescope (HST) by Benedict et al. (2007), Riess et al. (2014), and Casertano et al. (2016), respectively. The parallax of FF Aql was determined with the HST Fine Guidance Sensor, reaching a precision of $\sigma_{\varpi} / \varpi \sim 6 \%$. The astrometric measurements of SY Aur and SS CMa were obtained with the Wide Field Camera 3 (WFC3) by spatial scanning that improved the precision of the source position determination allowing parallaxes with uncertainties in the range of $\sim 0.3-0.5$ mas $\left(\sigma_{\varpi} / \varpi \sim 11-12 \%\right)$ to be derived. Parallax measurements available for these three stars are summarised in the upper portion of Table 1. Taking into account the rather small sample and the much larger errors, as expected for these first Gaia parallaxes, agreement between TGAS and HST is within $2 \sigma$ for FF Aql and SS CMa, and within $1 \sigma$ for SY Aur. We also note that FF Aql is known to be in a binary system and this may have affected the measure of its parallax (see Sect. 4.1). Figure 8 shows for these three classical Cepheids the comparison between the TGAS and HST parallax values (lower panel), between TGAS and HIPPARcos (middle panel), and between HIPPARCos and the HST (upper panel). Going from top to bottom the agreement between the different parallax values increases, the best agreement existing between the TGAS and HST values, thus confirming that TGAS, although less precise than HST, provides more reliable parallax measurements and an improvement with respect to HiPPARCos.

The parallax has been measured with the HST only for one of the Type II Cepheids in our sample, VY Pyx (Benedict et al. 2011). Results of the comparison between the TGAS, HIPPARCos, and HST parallaxes for this star are summarised in the middle portion of Table 1 and are shown in Fig. 9.
The TGAS parallax for VY Pyx differs significantly from the HST and HIPPARCos values, which, on the other hand, seem to be in reasonable agreement with each other. However, as discussed in Benedict et al. (2011) the $K$-band absolute magnitude of VY Pyx inferred from the HST parallax places the star 1.19 mag below the $P M_{K}$ relation defined by five RR Lyrae stars with parallax also measured by the HST (see Fig. 6 in Benedict et al. 2011, and the discussion below), in contrast with the Type II Cepheids being expected to lay on the extrapolation to longer periods of the RR Lyrae star $P M_{K}$ relation (see e.g. Ripepi et al. 2015, and references therein). Benedict et al. (2011) explain this discrepancy either as being due to the wide range in absolute magnitude spanned by the short-period Type II Cepheids or as being caused by some anomaly in VY Pyx itself. We have reproduced Fig. 6 from Benedict et al. (2011) in our Fig. 10, using for the five RR Lyrae stars in the Benedict et al. sample the $M_{K}$ magnitudes calculated on the basis of their TGAS parallaxes (blue filled circles) and plotting with red lines the $P M_{K}$ relations obtained using instead the Benedict et al. HST parallaxes for the five RR Lyrae stars with (red solid line) and without (red dashed line) Lutz-Kelker corrections (Lutz \& Kelker 1973). Green circles represent star VY Pyx with the $M_{K}$ magnitude calculated on the basis of the Benedict et al. HST parallax (open circle) and TGAS parallax (filled circles), respectively. The TGAS parallax makes VY Pyx nicely follow the $P M_{K}$ relation defined by the five RR Lyrae stars, both in the formulation based on their TGAS parallaxes (black solid line) and that based on the Benedict et al. parallaxes (red solid lines).

As anticipated in the discussion of VY Pyx, HST parallaxes have been measured by Benedict et al. (2011) for five RR Lyrae stars. The comparison between HIPPARCos, TGAS, and Benedict et al. (2011) for these five variables is summarised in the lower portion of Table 1 and graphically shown in Fig. 11 for HIPPARCos versus HST (upper panel), TGAS versus HIPPARCos (middle panel) and TGAS versus HST (lower panel). Errors on the HIPPARCOS parallaxes are much larger than those on the HST and TGAS measures and, except for RR Lyrae itself, the HIPPARCos parallaxes differ significantly from the HST values, whereas the TGAS and HST parallaxes agree within $1 \sigma$ for RR Lyr, SU Dra, UV Oct, and XZ Cyg. On the other hand, the $1 \sigma$ agreement of the HIPPARCOS, TGAS, and 

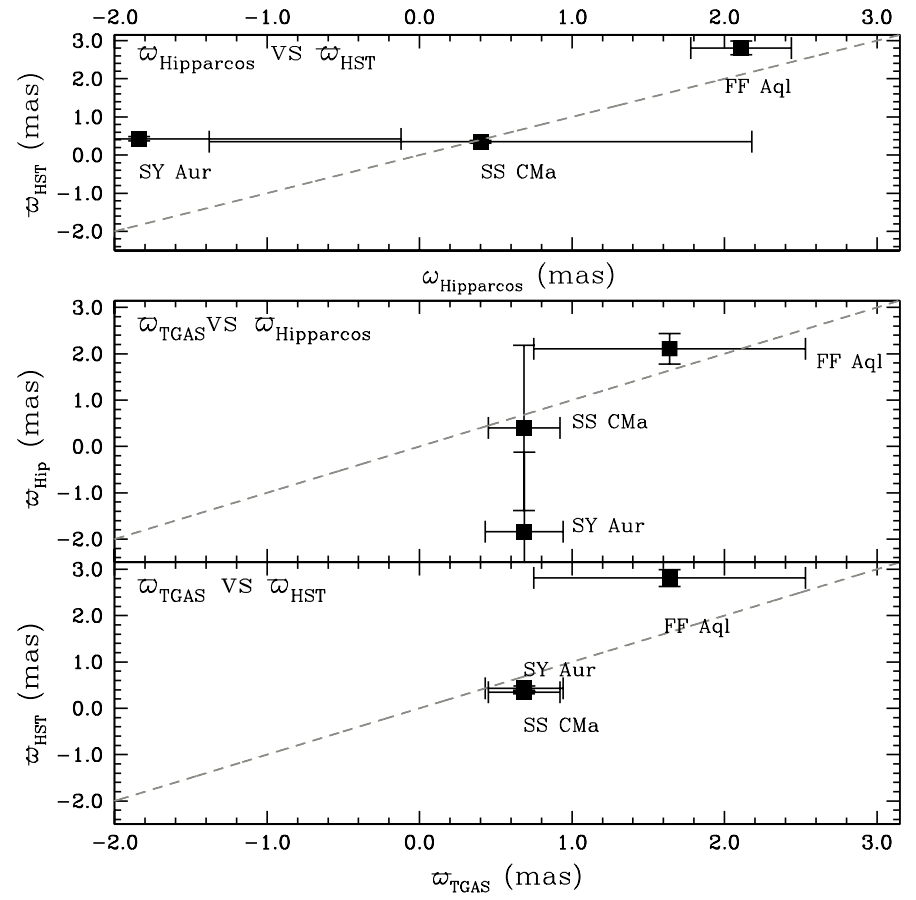

Fig. 8. Comparison between HIPPARCos and HST parallax (upper panel), TGAS and HIPPARCOS parallax (middle panel), and TGAS and HST parallax (lower panel) for the classical Cepheids FF Aql, SY Aur, and SS CMa. FF Aql is the brightest star in our sample of 331 classical Cepheids and is known to be a component of a binary system. A dashed line shows the bisector.

HST parallax values for RR Lyrae itself is particularly satisfactory, also in light of the much reduced error bar in the TGAS value: 0.23 mas compared with 0.64 mas in HIPPARCOS. For the remaining star, RZ Cep, Benedict et al. (2011) provide two different parallax values, 2.12 and 2.54 mas (Neeley et al. 2015). We show both values in Fig. 11. Although the Benedict et al. (2011) preferred value for this star is 2.12 mas (corresponding to the grey filled circle in Fig. 11), the alternative value of 2.54 mas is in much better agreement with the TGAS parallax of RZ Cep and nicely places the star on the bisector of the HST and TGAS parallaxes. To conclude, as already noted for the classical Cepheids (see Fig. 8), the best agreement is found between the TGAS and the HST parallaxes, confirming once again the higher reliability of the TGAS parallaxes and the improvement with respect to HIPPARCos.

Figure 10 deserves further comments. There is a systematic zero point offset of about 0.14 mag between the $P M_{K}$ relation inferred from the HST parallaxes of Benedict et al. (2011) for the five RR Lyrae stars without applying any Lutz-Kelker correction (red dashed line) and the relation (black solid line) obtained with the $M_{K}$ magnitudes inferred from the TGAS parallaxes (blue filled circles). The latter relation was obtained by linear least-squares fit of the $M_{K}$ magnitudes based on the TGAS parallaxes, adopting the same slope as in Benedict et al. (2011), that is -2.38 from Sollima et al. (2008) and without applying Lutz-Kelker corrections. Since there is good agreement between the TGAS and HST parallaxes of these five RR Lyrae stars, the observed zero point offset between $P M_{K}$ relations might hint to some systematic effect in the method used to compute these relations. Indeed, as discussed in detail in Sect. 3.2, the direct transformation of parallaxes to absolute magnitudes and linear leastsquares fits is not advisable in the presence of large errors like
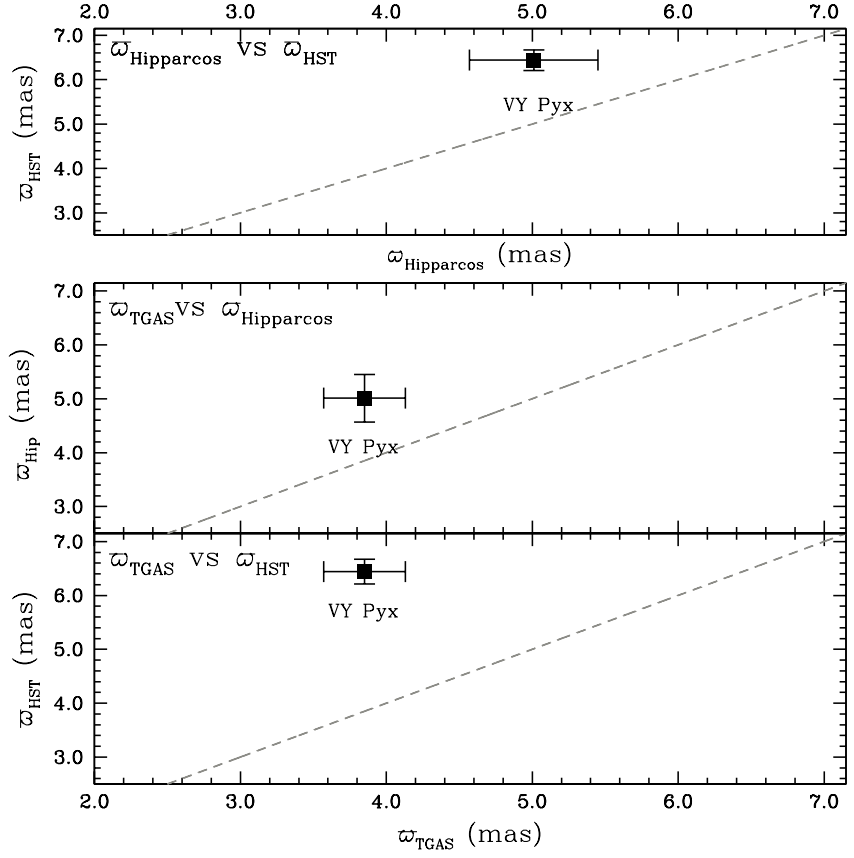

Fig. 9. Comparison between HiPPARcos and HST parallax (upper panel), TGAS and HIPPARCos parallax (middle panel), and TGAS and HST parallax (lower panel) for the Type II Cepheid VY Pyx. Dashed lines show the bisectors.

those affecting the parallaxes of these stars, and this might have induced systematic effects.

We note that although globally the possible systematic errors in the TGAS parallaxes are well below their formal errors ${ }^{5}$, there could still be some systematic effects at a typical level of \pm 0.3 mas depending on the sky position and the colour of the source (Lindegren et al. 2016). However, the question of these additional systematic errors is still very much under debate within DPAC, and its value has often been recognised as an overestimate, which is why uncertainties smaller than 0.3 mas can be found in the TGAS catalogue. In principle, the nominal uncertainties quoted in the TGAS catalogue already contemplate all sources of variance including the systematic uncertainties and a safety margin. Therefore, there should be no need to add the 0.3 mas extra-variance. Furthermore, the zero point error in the parallaxes is of the order of -0.04 mas (Arenou et al. 2017), hence does not seem to support the need for the extra-variance. Additionally, while the analysis of regional/zonal effects (for example in quasars) shows differences across various regions of the sky, these systematic effects are spatially correlated and not totally random over the celestial sphere. Hence, they become an important issue only if analysing a particular region of the sky, like star clusters. However, in all-sky studies like those presented in this paper, and particularly for the RR Lyrae stars, which are not concentrated in any specific part of the sky (see Fig. 4) this systematic effect does not influence the global zero point of the derived $P L, P L Z$, and $M_{V}-[\mathrm{Fe} / \mathrm{H}]$ relations.

Arenou et al. (2017) report systematic zero points respectively of $-0.014 \pm 0.014$ mas and $-0.07 \pm 0.02$ mas in the TGAS parallaxes of 207 classical Cepheids and 130 RR Lyrae stars they have analysed, and an average shift of $-0.034 \pm 0.012$ mas when combining the two samples. We have not found information in the literature about systematic effects on the HST parallaxes.

5 Casertano et al. (2017) claim that formal errors of TGAS parallaxes may also be overestimated. 


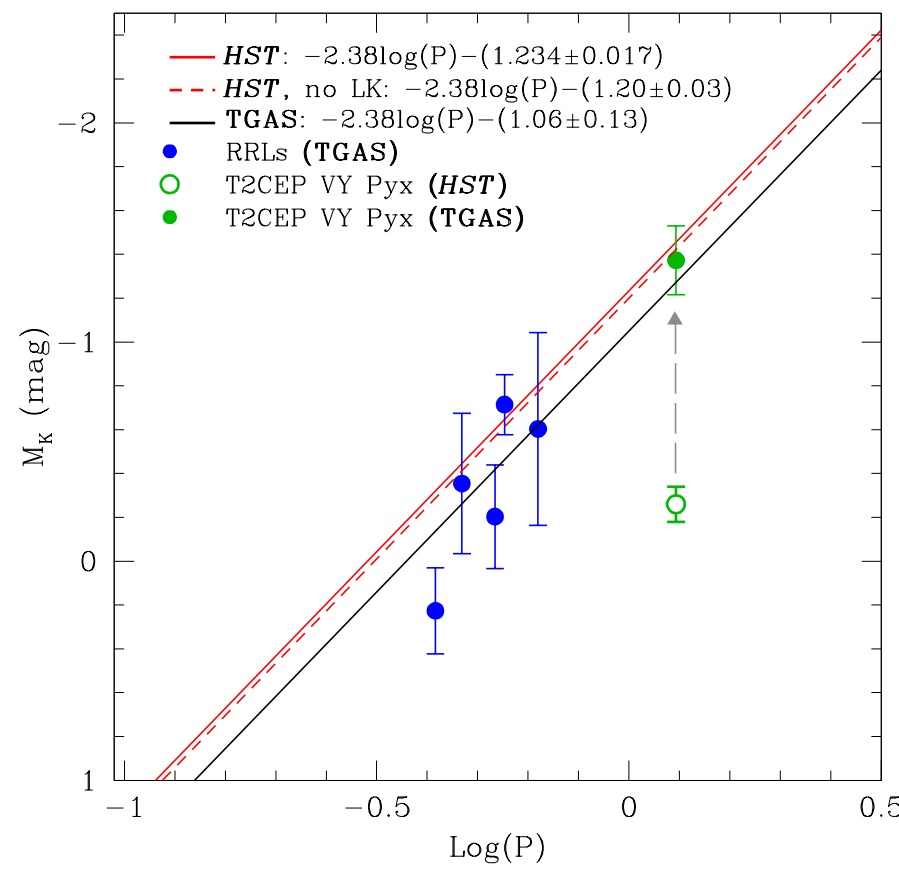

Fig. 10. Weighted linear least-squares fit performed over the $M_{K}$ magnitude of the five RR Lyrae stars in Benedict et al. (2011) using the $M_{K}$ values inferred from the HST parallaxes with (red solid line) and without (red dashed line) Lutz-Kelker correction and the $M_{K}$ values (blue filled circles) inferred from the TGAS parallaxes (black line). Green filled and open circles show the Type II Cepheid VY Pyx with the $M_{K}$ magnitude determined from the TGAS and HST parallax, respectively. The star was not used in the fit.

Nevetheless, the direct star-by-star comparison of the parallaxes in Table 1 and Fig. 11 does not seem to show evidence of the presence of a systematic difference between the TGAS and HST parallaxes of the fairly small sample (3 classical Cepheids, 1 Type II Cepheid, and 5 RR Lyrae stars) for which a direct comparison with the HST is possible.

\subsection{Comparison with parallaxes inferred by theoretical model fitting of the light curves}

An independent method for inferring the distance (hence the parallax) of a pulsating star is the "model fitting" of the multi-wavelength starlight curves through non-linear convective pulsation models (see e.g. Marconi \& Clementini 2005; Keller \& Wood 2006; Marconi et al. 2013a,b, and references therein). Indeed, one of the advantages of non-linear hydrodynamical codes that involve a detailed treatment of the coupling between pulsation and convection is that they are able to predict the variation of any relevant quantity along the pulsation cycle. The direct comparison between observed and predicted light curves based on an extensive set of models with the period fixed to the observed value but varying the mass, the luminosity, the effective temperature, and the chemical composition allows us to obtain a best fit model and in turn to constrain not only the distance, but also the intrinsic stellar properties of the pulsating star under study. This approach was first applied to a Magellanic classical Cepheid (Wood et al. 1997) and a field RR Lyrae (Bono et al. 2000), and later extended to cluster members (Marconi \& Degl'Innocenti 2007; Marconi et al. 2013b) and variables for which radial velocity curves were also available (see e.g. Di Fabrizio et al. 2002; Natale et al. 2008; Marconi et al. 2013a,b, and references
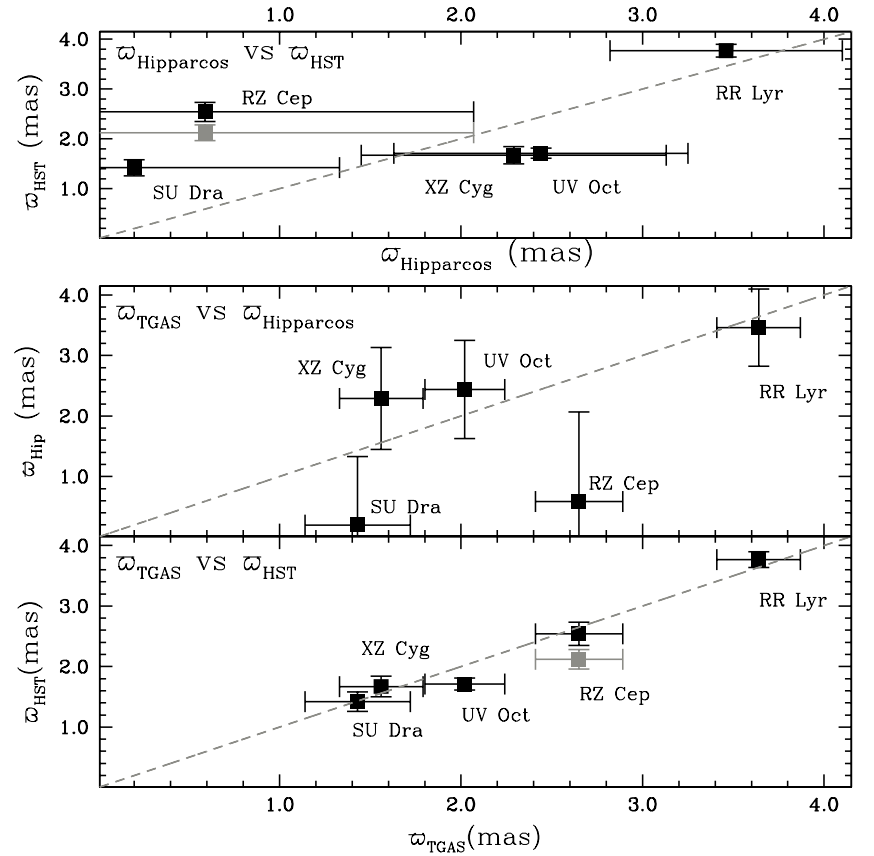

Fig. 11. Comparison between HIPPARCos and HST parallaxes (upper panel), TGAS and HIPPARCOS (middle panel), TGAS and HST (lower panel) for the RR Lyrae stars RR Lyr, RZ Cep, SU Dra, XZ Cyg, and UV Oct. Two values from Benedict et al. (2011) are shown for RZ Cep: 2.12 mas (grey filled square) and 2.54 mas (black filled square). TGAS parallax for RZ Cep is in good agreement with the larger, less favoured value in Benedict et al. (2011). Dashed lines show the bisectors.

therein). Furthermore, the method was successfully applied to a number of different classes of pulsating stars in the LMC (see e.g. Bono et al. 2002; Marconi \& Clementini 2005; McNamara et al. 2007; Marconi et al. 2013a,b, and references therein), also by different teams (see also Wood et al. 1997; Keller \& Wood 2002, 2006), but always obtaining consistent results. Because of the significant amount of time and computing resources required by the model fitting technique, here we applied this method only to three classical Cepheids, for which multi-band light curves are available in the literature, that we selected from the sample of classical Cepheids we used to derive the $P L$ relations in Sect. 4.2. The first case is RS Pup, pulsating in the fundamental mode with a period of 41.528 days. The photometric data for this star and for the other two analysed in this section are taken from a number of papers (Welch et al. 1984; Laney \& Stobie 1992; Berdnikov 2008; Monson \& Pierce 2011) and sample well the light variations in the different filters. Fig. 12 shows the results of model fitting the starlight curves in the $B, V, R, I, K$ bands. RS Pup is the second longest period classical Cepheid in our sample and is known to be surrounded by a nebula reflecting the light from the central star (see e.g. Kervella et al. 2008, 2014), thus allowing an independent geometric evaluation of the distance to be obtained from the light echoes propagating in the star circumstellar nebula, corresponding to a parallax $\varpi_{K 14}=0.524 \pm 0.022$ mas (see Kervella et al. 2014 , for details). This value is consistent within the errors with the TGAS value $\varpi_{\text {TGAS }}=0.63 \pm 0.26$ mas. The pulsation model best reproducing RS Pup multi-filter light curve corresponds to a $9 M_{\odot}$ star with an intrinsic luminosity $\log L / L_{\odot}=4.19$. From the apparent distance moduli obtained with the best fit in the various bands, we were able to estimate the extinction correction and the intrinsic distance modulus $\mu_{0}($ FIT $)=11.1 \pm 0.1 \mathrm{mag}$. This 
Teff=4875, $\log (\mathrm{L} / \mathrm{Lo})=4.19, \mathrm{M} / \mathrm{Mo}=9$, alpha=1.5

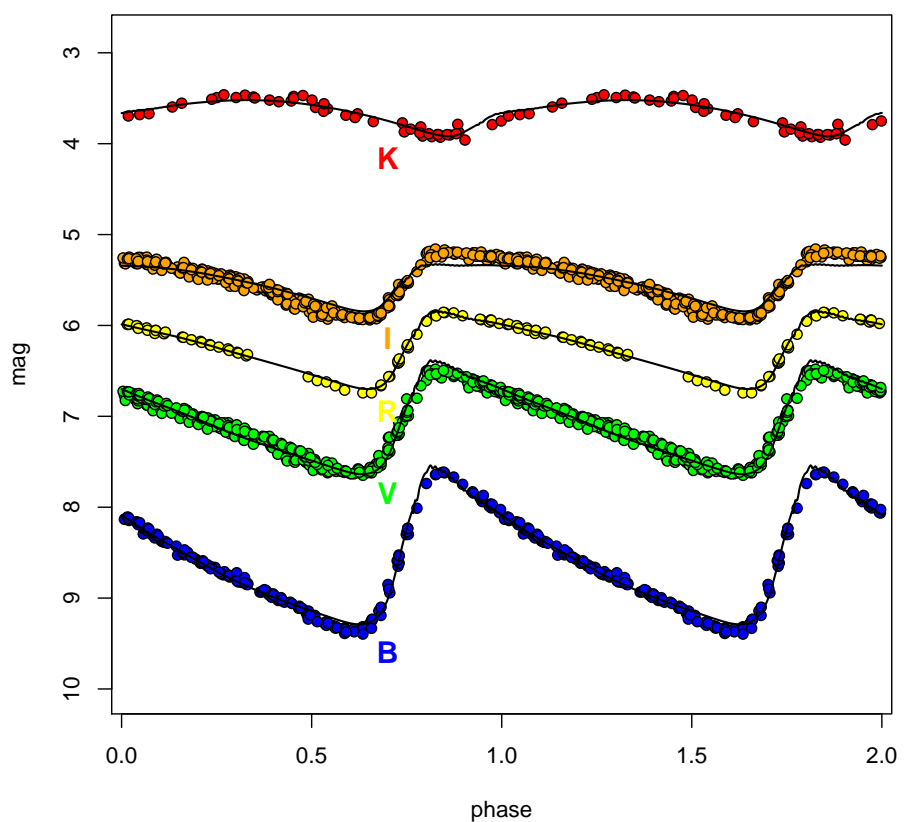

Fig. 12. Model fitting of the fundamental mode classical Cepheid RS Pup, the second longest period Cepheid in our sample with $P=$ 41.528 days. The model fitting provides a parallax of $\varpi_{\mathrm{FIT}}=0.58 \pm$ 0.03 mas in excellent agreement with the TGAS parallax for this star, $\varpi_{\mathrm{TGAS}}=0.63 \pm 0.26$ mas.

provides a model fitting parallax $\varpi_{\text {FIT }}=0.58 \pm 0.03$ mas, which is in excellent agreement with the TGAS parallax for the star.

Similarly, we performed the model fitting of V1162 Aql light curves, a Galactic fundamental mode Cepheid of much shorter period (5.376 days), as shown in Fig. 13. In this case, the pulsation model best reproducing the multi-filter light curve corresponds to a $5 M_{\odot}$ star with an intrinsic luminosity $\log L / L_{\odot}=3.26$. The inferred model fitting intrinsic distance modulus is $\mu_{0}(\mathrm{FIT})=10.5 \pm 0.1$, corresponding to a parallax of $\varpi_{\text {FIT }}=0.79 \pm 0.04$ mas, which is in agreement with the TGAS value $\left(\varpi_{\mathrm{TGAS}}=1.01 \pm 0.29\right.$ mas $)$, within the errors.

The third classical Cepheid analysed with the model fitting technique is RS Cas, a Galactic fundamental mode Cepheid with a period of about 6.296 days. When applying our model fitting approach, we obtain the best fit model shown in Fig. 14, corresponding to a $6 M_{\odot}$ star with an intrinsic luminosity $\log L / L_{\odot}=$ 3.38. This implies an intrinsic distance modulus $\mu_{0}(\mathrm{FIT})=$ $11.1 \pm 0.1$ and a pulsation parallax of $\varpi_{\mathrm{FIT}}=0.60 \pm 0.03 \mathrm{mas}$, much smaller than the TGAS parallax of $\varpi_{\mathrm{TGAS}}=1.53 \pm$ 0.32 mas. Consequently, the predicted distance modulus is about 2 mag longer than the TGAS-based value, and the absolute magnitude is brighter by the same amount. It is interesting to note that an upward shift of approximately 1.5-2 mag would allow RS Cas to match the $P L$ relations in Fig. 21. This seems to suggest that the TGAS parallax for RS Cas is incorrect. We wonder whether the discrepancy observed for this star may be caused by a companion such as a white dwarf, which might affect the TGAS measurement.

Finally, we note that the theoretical model fitting technique has also been applied to RR Lyrae stars both in and outside the MW (e.g. Bono et al. 2000; Di Fabrizio et al. 2002; Marconi \& Clementini 2005). For one of the RR Lyrae stars with TGAS parallax, U Com, Bono et al. (2000) measured the parallax by fitting the star multi-band light curves with
Teff=5750, $\log (\mathrm{L} / \mathrm{Lo})=3.26, \mathrm{M} / \mathrm{Mo}=5$, alpha=1.5

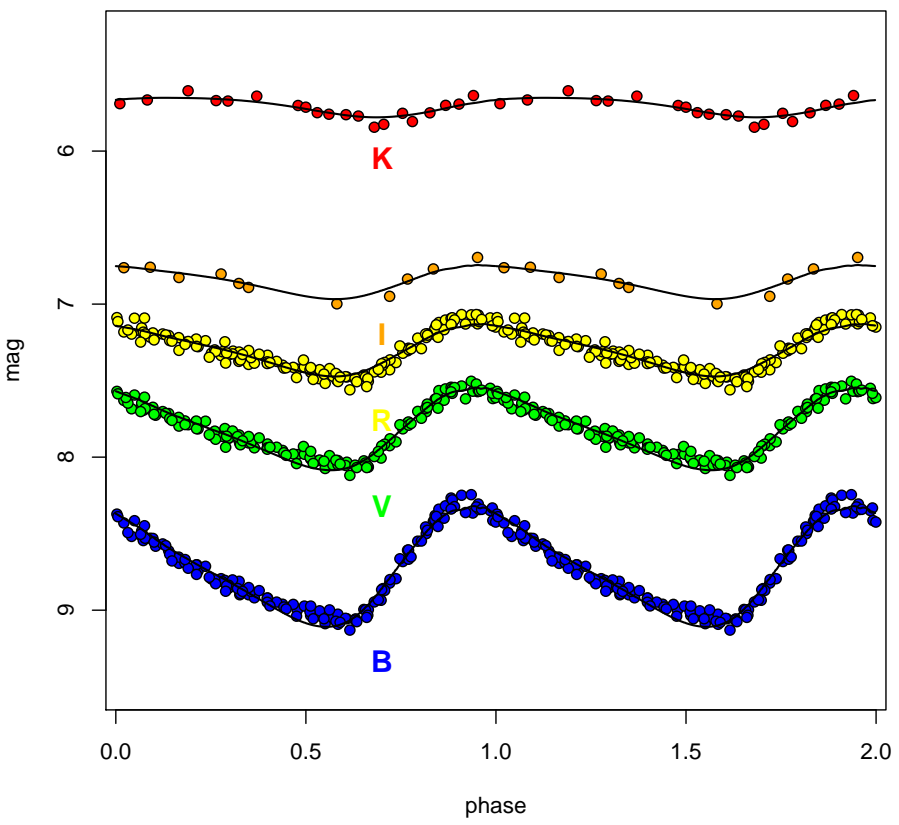

Fig. 13. Model fitting of the fundamental mode classical Cepheid V1162 Aql, $P=5.376$ days. The model fitting provides a parallax of $\varpi_{\text {FIT }}=0.79 \pm 0.04$ mas in agreement, within the errors, with the TGAS parallax for this star, $\varpi_{\mathrm{TGAS}}=1.01 \pm 0.29$ mas.

Teff=5675, $\log (\mathrm{L} / \mathrm{LO})=3.38, \mathrm{M} / \mathrm{Mo}=6$, alpha $=1.3$

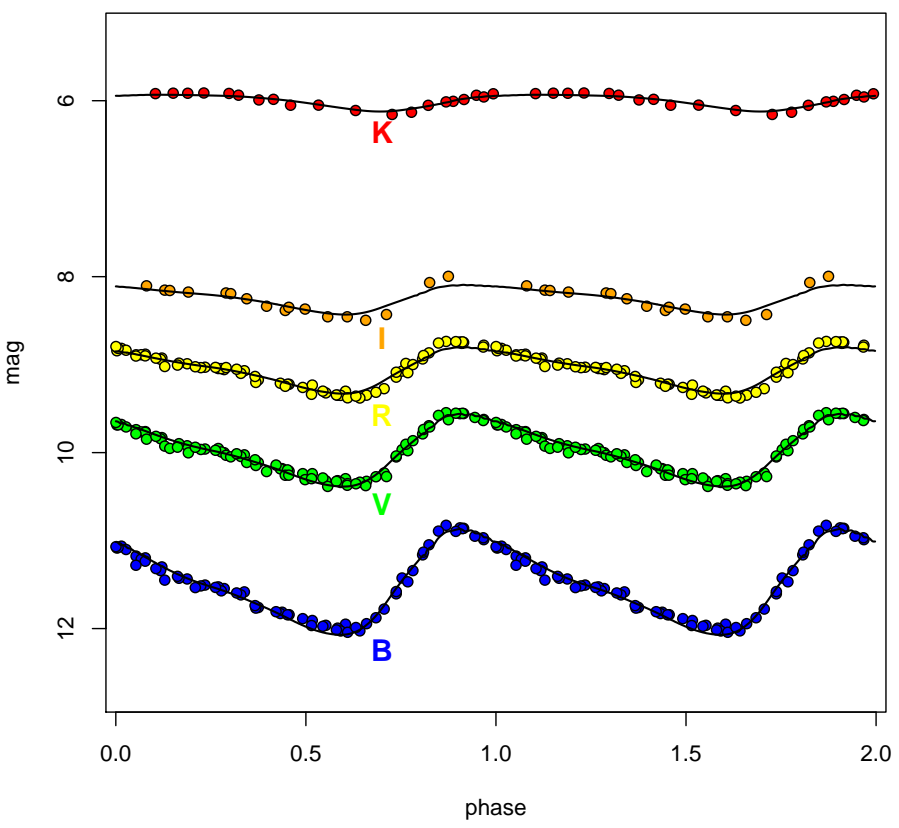

Fig. 14. Model fitting of the fundamental mode classical Cepheid RS Cas, $P=6.296$ days. The model fitting provides a parallax of $\varpi_{\mathrm{FIT}}=$ $0.60 \pm 0.03$ mas in significant disagreement with the TGAS parallax for this star, $\varpi_{\mathrm{TGAS}}=1.53 \pm 0.32$ mas.

non-linear convective pulsation models: $\varpi_{\mathrm{FIT}}=0.63 \pm 0.02$ mas. This value agrees, within the errors, with TGAS parallax for $\mathrm{U}$ Com: $\varpi_{\mathrm{TGAS}}=0.46 \pm 0.28$ mas.

The results presented in this section confirm the predictive capability of the adopted theoretical scenario and the potential 


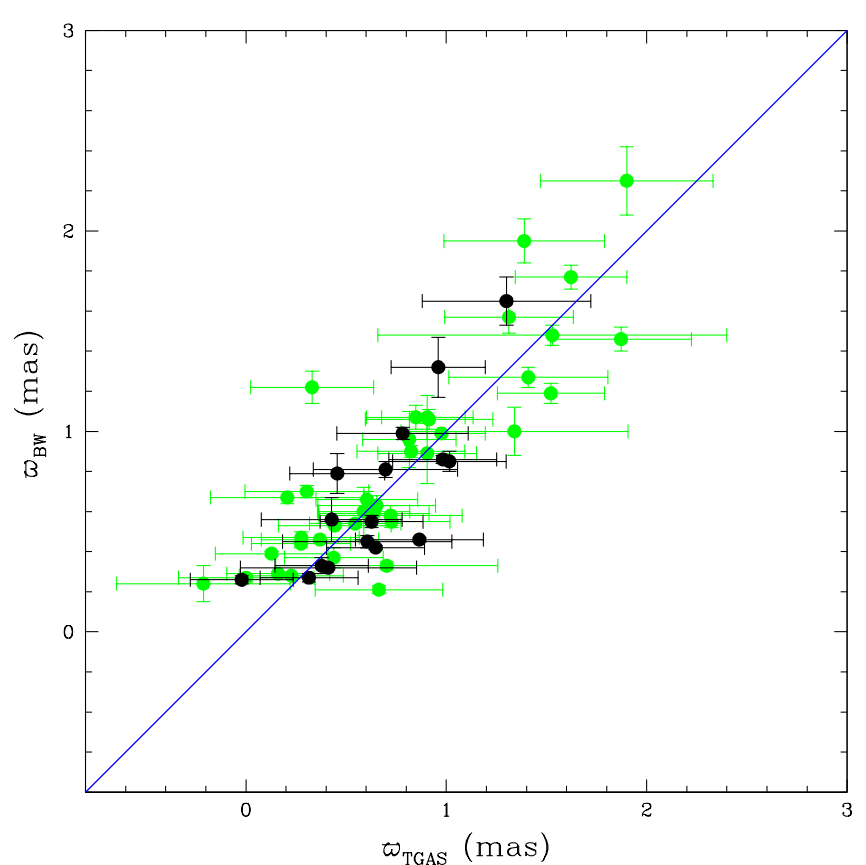

Fig. 15. Comparison between the photometric parallaxes estimated via the B-W method and the TGAS parallaxes of classical Cepheids. Black and green circles represent single and binary Cepheids, respectively. The blue line is the bisector.

of the light curve model fitting technique to test and constrain the accuracy of empirical distance determinations.

\subsection{Comparison with Baade-Wesselink studies.}

Photometric parallaxes of classical Cepheids and RR Lyrae stars have often been estimated with the Baade-Wesselink (B-W) method in various different implementations (e.g. the Infrared Surface Brightness technique or the Spectro-PhotoInterferometric modelling approach of Mérand 2015 and Breitfelder et al. 2016).

Fouqué et al. (2007) list in their Table 6 the photometric parallaxes inferred from the application of the Infrared Surface Brightness version of the B-W technique for 62 classical Cepheids, 54 of which have a TGAS parallax estimate. The comparison between the TGAS and the B-W parallaxes for these 54 Cepheids is shown in Fig. 15. The sample of 54 classical Cepheids contains a large fraction (38) of binary systems. The phenomenon of binarity/multiplicity is rather common among classical Cepheids, as will be discussed in more detail in Sect. 4.1. The presence of a binary companion may prevent an accurate estimate of parallax. Cepheids known to be in binary systems are shown as green circles in Fig. 15; they are more scattered around the bisector line. The rms scatter from the bisector line is 0.28 mag for binary classical Cepheids and reduces to 0.23 mag when the 16 non-binary Cepheids are considered. A weighted least-squares fit of the relation $\varpi_{B-W}=\alpha \varpi_{\mathrm{TGAS}}$ returns a slope value of $(0.90 \pm 0.07)$ for the sample of 16 classical Cepheids which are not in binary systems.

A similar comparison was also done for the RR Lyrae stars. We considered 19 MW RR Lyrae variables with TGAS parallaxes and absolute visual $\left(M_{V}\right)$ and $K$-band $\left(M_{K}\right)$ magnitudes available in the literature from B-W studies (see Table 2 in Muraveva et al. 2015). The B-W absolute magnitudes were taken from the compilations in Table 11 of Cacciari et al. (1992) and Table 16 of Skillen et al. (1993) and revised, first assuming

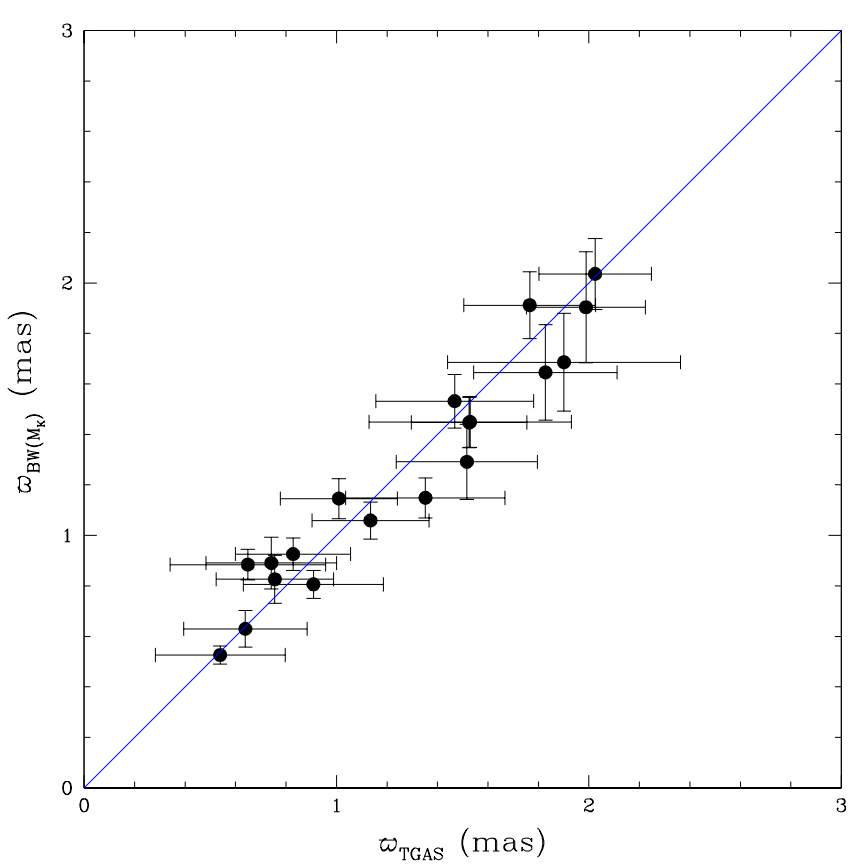

Fig. 16. Comparison between the photometric parallaxes inferred from the $K$-band absolute magnitudes $\left(M_{K}\right)$ estimated via the B-W method and the TGAS parallaxes for RR Lyrae stars. The blue line represents the bisector.

for the $p$ factor used to transform the observed radial velocity to true pulsation velocity the value $p=1.38$ proposed by Fernley $(1994)^{6}$, and then averaging multiple determinations of individual stars. We note that the $K$-band magnitudes of RR Lyrae stars analysed with the B-W method are in the Johnson photometric system; however, the difference between the 2MASS $K_{\mathrm{s}}$ and the Johnson $K$ is small, about 0.03 mag on average, for the typical colour of RR Lyrae stars (Muraveva et al. 2015) and is always much smaller than the individual errors in the B-W $M_{K}$ magnitudes. We have transformed the B-W absolute magnitudes to photometric parallaxes. The direct transformation of parallaxes to absolute magnitudes and vice-versa should be avoided when parallax uncertainties are large because of the resulting asymmetric errors (see Sect. 3.2). However, given the small relative errors in distance moduli (less than $3 \%$ ) of the 19 RR Lyrae stars analysed with the B-W technique, the uncertainties in the inferred parallaxes are symmetric and would not be affected by any reasonable prior distribution. Comparison of the photometric parallaxes inferred from the $M_{K}$ absolute magnitudes of the 19 RR Lyrae stars with the corresponding TGAS parallaxes is shown in Fig. 16. The two independent parallax estimates appear to be in very good agreement within the errors. We also performed the comparison with the parallaxes inferred from the $V$-band absolute magnitudes. A weighted least-squares fit of the relations $\varpi_{M_{V}(B-W)}=\alpha \varpi_{\mathrm{TGAS}}$ and $\varpi_{M_{K}(B-W)}=\alpha \varpi_{\mathrm{TGAS}}$ returns slopes of 0.97 and 0.98 , respectively, which are both very close to the bisector slope $\alpha=1$. To conclude, TGAS parallaxes are in general good agreement with the photometric parallaxes obtained in the B-W studies of classical Cepheids and RR Lyrae stars. The agreement is particularly good for the RR Lyrae stars and seems to support the adoption of the larger $p$ factor proposed by Fernley (1994).

6 According to Table 1 in Fernley (1994), this change in the $p$ factor makes the B-W absolute magnitudes become systematically brighter by $0.1 \mathrm{mag}$ on average. 


\section{Selection biases and methods}

\subsection{Biases}

When determining a luminosity calibration (e.g. a $P L, P W, P L Z$, or $M_{V}-[\mathrm{Fe} / \mathrm{H}]$ relation in our case) from astrometric data, we have to be very careful by taking into account possible sources of bias that can affect it lest the results contain systematic errors. A first source of such biases is the way the sample has been obtained, due to either censorship (missing data) or to truncation (some selection process done on purpose for the study). Selection criteria that directly or indirectly favour brighter or fainter stars can affect the $P L, P W, P L Z$, or $M_{V}-[\mathrm{Fe} / \mathrm{H}]$ relations derived from the sample. Specifically, the so-called Malmquist bias (Malmquist 1936) caused by the limitation in apparent magnitude of the TGAS subset has to be taken into account. In this section we attempt to discuss qualitatively the effects of the different selection filters that result in the samples used in this work to infer the luminosity calibration. It is well known that biased samples may result in biased estimates of the (linear) model parameters. Our samples are the result of several processing stages each with a different impact on the resulting sample. As it turns out, the very limited size of our samples and the magnitude of the uncertainties mask all of these effects.

\subsubsection{Trucations/censorships in the generation of the TGAS catalogue}

We first discuss the truncation of the samples at the bright end. Stars brighter than $B_{T}=2.1$ or $V_{T}=1.9$ mag were excluded from the Tycho-2 catalogue. In particular, 17588 stars included in the HIPPARCOS catalogue were not included in Tycho-2. Some of these were actually included in TGAS, but TGAS is itself affected by the removal of many (but not all) sources brighter than $G \sim 7 \mathrm{mag}$, so the effects of the truncation are subtle and difficult to assess. We have checked the existing catalogues to identify known Tycho-2 classical Cepheids and RR Lyrae stars missing from the TGAS catalogue. It turns out that 54 known RR Lyrae stars and 57 classical Cepheids in Tycho-2 are missing from the TGAS catalogue. Only two Type II Cepheids are missing in the TGAS catalogue, hence in the following we focus on RR Lyrae star and classical Cepheid samples and do not discuss Type II Cepheids further. The distributions in apparent $G$ magnitude of the missing RR Lyrae stars and Cepheids are shown in Fig. 17. We see that the truncation at the bright end of the Tycho-2 sample does not affect the RR Lyrae sample, while it results in the loss of 24 classical Cepheids. This represents a major loss that can seriously affect the inferences of the $P L$ and $P W$ relations for classical Cepheids. Fortunately, 21 of these 24 sources are also in the HIPPARCos catalogue and we can gauge its impact in the inference. It turns out that the HIPPARCOS parallaxes and periods are fully consistent with the distributions from our TGAS sample both in the 2D plane and in their marginal distributions. We can therefore conclude that this loss did not bias our sample in any respect.

High proper motion $(\mu>3.5$ arcsec/yr) Tycho- 2 stars are also missing from the TGAS catalogue, although this selection has no effect on our samples, at least to the level that can be checked with Tycho-2 proper motions (i.e. the Tycho-2 proper motions of the known RR Lyrae and classical Cepheids missing from TGAS are well below the limit of $3.5 \mathrm{arcsec} / \mathrm{yr})$. So we can discard this truncation as a potential source of biases.

As discussed in Gaia Collaboration (2016a), the Gaia DR1 has completeness issues in dense areas of the sky where the

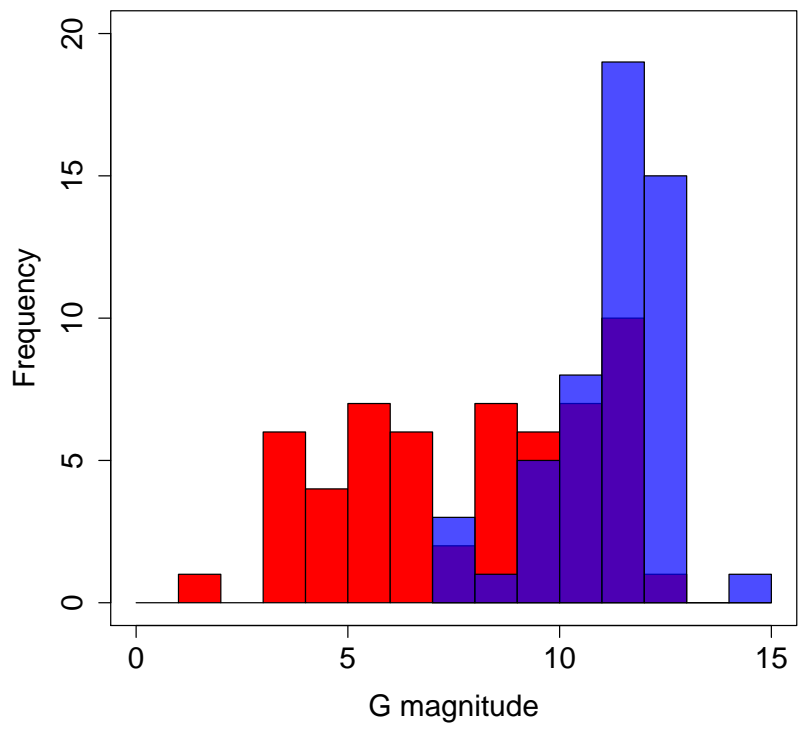

Fig. 17. Apparent magnitudes of known Tycho-2 RR Lyrae stars (blue) and classical Cepheids (red) not included in the TGAS catalogue.

limiting magnitude of the catalogue can be brighter by several magnitudes. This could result in a potential loss of Cepheids in dense regions of the disc, were it not for the bright limiting magnitude of the Tycho-2 catalogue. RR Lyrae stars are not concentrated in the disc (see Fig. 4) and therefore are even less likely to be affected.

Another truncation/censorship of the Tycho-2 sample comes from the rejection by the photometric processing of sources with less than five transits or extreme red or blue colour indices. The cut in the number of transits results in a loss of sources with projected positions near the ecliptic that should not bias our samples in any respect. Biases will appear if there is a correlation between the position in the celestial sphere and the brightness of the sources, such that the sources missed due to an insufficient number of transits were predominantly bright or faint (we discard direct correlations with the period). In principle, the number of transits depends on the scanning law and so any correlation, if present, should be negligible.

The selection of sources based on the estimated $G_{B P}-G_{R P}$ can be very feasibly reduced to the rejection of the sources outside the range $[0.5,3.5]$ mag (but for details see Gaia Collaboration 2016a). This may result in a loss of stars in the highest extinction regions of the disc. If periods are not taken into account and only the instability strip is considered, the brighter stars will be redder and thus more prone to be rejected by the photometric reduction pipeline, due to interstellar reddening beyond the 3.5 mag limit. In principle, this should only significantly affect the sample of Cepheids (because RR Lyrae stars are not concentrated in the disc where most of the extinction occurs). For these samples of brightest sources and a fixed period, however, the brighter sources are at the blue edge of the instability strip, so at any given period in the $P L, P W$, or $P L Z$ relations, we are more likely to lose the fainter stars. In summary, we may expect a bias present in the bright part of the $P L$ relations in the sense of an underrepresentation of the fainter pulsators.

We have estimated the $G, G_{B P}$, and $G_{R P}$ magnitudes for Tycho-2 sources missing from TGAS using the photometric relations by Jordi et al. (2010). Figure 18 shows the distribution of estimated $G_{B P}-G_{R P}$ colour indices for the samples of classical Cepheids (red) and RR Lyrae stars (blue). As expected, all values are bluer than the $3.5 \mathrm{mag}$ limit and hence reddening is 


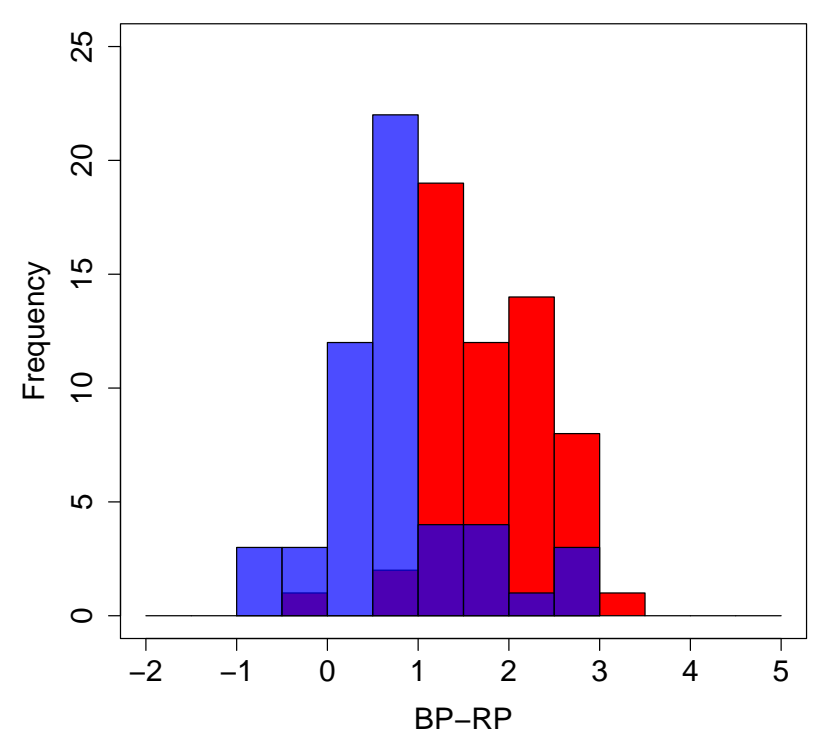

Fig. 18. Estimated $G_{B P}-G_{R P}$ colour indices of known Tycho-2 RR Lyrae stars (blue) and classical Cepheids (red) missing from the TGAS catalogue.

not responsible for the exclusion of this sources from the catalogue. On the other hand, however, the TGAS catalogue misses at least 16 RR Lyrae-type pulsators (but only one Cepheid) with colour indices bluer than 0.5 . This should more strongly affect the fainter members of the instability strip, which are bluer on average.

Finally, sources with astrometric uncertainties beyond 1 mas/yr in proper motion, 20 mas in position, or 1 mas in parallax are also excluded from the TGAS catalogue. All these uncertainties depend on the source colour and apparent magnitude, and the complex TDI-gating scheme makes a more detailed quantification of the effects very difficult. In general, the fainter and/or redder the star, the larger the uncertainty, with the apparent magnitude having a much stronger effect on the uncertainties (see e.g. de Bruijne et al. 2005). We see from simulations that the net effect is a bias that predominantly affects the long-period end of the $P L, P W$, or $P L Z$ distributions in the sense of shifting the average absolute magnitude for a fixed period towards brighter (more negative) values. Hence, it has the opposite effect of the Malmquist bias, which tends to flatten the slope of the $P L$ relations. Indeed, we see from these simulations that in some cases, the two biases cancel their effects on the slope and only a zero point shift remains as the net result of the two biases.

\subsubsection{Selections caused by availability of external data}

So far, we have analysed the effects of the Malmquist bias already present in the Tycho- 2 sample, and the biases emerging from the various truncations/censorships of the Tycho-2 sample carried out in the generation of the TGAS catalogue. In this work we apply a last filter to retain only sources in TGAS with $V, I, J$, $K_{\mathrm{s}}$, or $W 1$ magnitudes and periods available (hereafter external data; see Sects. 4-6). Therefore, the effect of this last filter remains to be discussed. If the filter left the distribution of TGAS pulsators unchanged, then no further bias would be introduced in the analyses. In the following paragraphs we study the differences under the light of the available data.

We have compared the 1D empirical distributions in parallax, absolute magnitudes, and periods (with respect to OGLE distributions of the LMC fundamental mode classical Cepheids and the LMC and Galactic bulge RR Lyrae stars). In the following, we apply the Anderson-Darling test (Anderson \& Darling 1952) which is a frequentist approach to a problem that would be better approached using Bayesian methods. Unfortunately, the frequentist approach (with all its limitations) will have to suffice until we can put forward a reasonable parametric model of the biases.

For the RR Lyrae parallaxes, the Anderson-Darling test yields a $p$-value of 0.00008 , well below a reasonable significance level. The main difference between the two distributions is a clear excess of parallaxes greater than 2 mas in the sample of TGAS sources without external data. When the uncertainties are taken into account using bootstrapping, the net result is that $42 \%(80 \%)$ of the experiments fall below the common 0.01 (0.05) significance level.

If we remove sources with negative parallaxes, we can apply the Anderson-Darling test to check whether the two samples of absolute magnitudes could have been drawn from the same distribution. The result is a $p$-value equal to $9 \times 10^{-7}$; the set of stars without external data show a median absolute $G$ magnitude $\left(1.2_{-0.7}^{+0.8}\right.$ with the uncertainties quoting the first to third quantile range) fainter than the sample used for the $P L$ relations $(0.7 \pm 0.5)$. The bootstrapping experiment performed to include the uncertainties in the analysis yields $38 \%(68 \%)$ of experiments with values below the $0.01(0.05)$ significance level.

In both cases (parallaxes and absolute magnitudes), the evidence for a difference seems inconclusive when the uncertainties are taken into account, but there are very significant hints that indicate an underrepresentation of faint sources in the TGAS sample. This evidence is made more robust if we re-estimate the $p$-values using absolute magnitudes derived from distance estimates obtained along the lines suggested by Bailer-Jones (2015). In this case we use the prior for the distance derived from the Bayesian models of the PL relationships (in the hierarchical model, this prior is inferred as part of the model, as explained in Sect. 3.2). The final $p$-value obtained with these absolute magnitudes is 0.003 , again below the two significance levels quoted above.

For the parallaxes of classical Cepheids, the AndersonDarling test yields a $p$-value of 0.01 . In this case again, the difference is the excess of large parallaxes in the sample of sources without external data with respect to the TGAS sample. The excess is much smaller than in the RR Lyrae case. When the uncertainties are taken into account, $11 \%(45 \%)$ of the experiments fall below the $0.01(0.05)$ significance level.

For absolute magnitudes (and again, removing stars with negative parallaxes) we obtain a $p$-value of 0.22 (without bootstrapping the uncertainties) and $0.02 \%(0.002 \%)$ of the bootstrap samples below the 0.01 (0.05) significance level. Hence, if the difference is real, we do not expect it to bias our $P L$ inferences significantly.

Overall, the complexity of the censorships of the samples available in this paper is not easy to interpret and makes it difficult to produce a reliable estimation of all possible biases introduced by them. We have identified at least three clear sources of biases: (i) the loss of the bluest RR Lyrae stars; (ii) the absence in the Gaia DR1 of the sources with the largest astrometric uncertainties (i.e. the exclusion from the TGAS catalogue of sources with uncertainties larger than 1 mas/yr in proper motion, 20 mas in position, or 1 mas in parallax); and (iii) the selection of sources with external data available. Although we have assumed that the local systematic correlations described in Sect. 2.2 average out for the two stellar types discussed, the lack of a detailed description of these correlations prevents us 
from completely discarding potential biases in the case of classical Cepheids which are not distributed uniformly in the celestial sphere. The results presented in this paper should be considered as preliminary; they will be superseded by the results from further releases of Gaia data allowing the use of samples less affected by uncontrolled censorship effects.

\subsection{Methods}

A commonly used way to obtain the luminosity calibration (e.g. a $P L, P W, P L Z$, or $M_{V}-[\mathrm{Fe} / \mathrm{H}]$ relation) is the direct transformation to distance (and then absolute magnitude) by parallax inversion and then the least-squares fit of the derived parameters. However, in the presence of parallaxes with errors larger than $10 \%$ this method has significant disadvantages.

The absolute magnitude of a star is calculated from its parallax by using the relation

$M=m_{0}+5 \log (\varpi)-10$,

where $M$ is the absolute magnitude, $m_{0}$ is the apparent dereddened magnitude of the star, and $\varpi$ is the parallax in mas. Although the errors in the Gaia parallaxes are well behaved, approximately Gaussian, and thus symmetrical, it is important to note that the errors in these derived magnitudes are not. The logarithm in the expression makes the derived error in $M$ asymmetrical and can thus lead to biases. In particular, the application of the least-squares method to a fit using these values is generally not advisable since this method relies on the errors of the fitted values being Gaussian or at least symmetrical. Furthermore, negative parallaxes cannot be used in such fitting processes.

In order to include stars with negative parallax measurements and to avoid non-linear transformations when fitting the $P L, P W$, $P L Z$, or $M_{V}-[\mathrm{Fe} / \mathrm{H}]$ relations, we follow the prescription of Arenou \& Luri (1999), who define an Astrometry-Based Luminosity (ABL) as

$a=10^{0.2 M}=\varpi 10^{0.2 m_{0}-2}$,

where $M$ is the absolute magnitude, $\varpi$ the parallax in mas, and $m_{0}$ the extinction corrected apparent magnitude. Using the ABL instead of the absolute magnitude is preferable as the parallax is used linearly, leading to symmetrical error bars, as occurs for the parallax errors. Furthermore, there is no additional Lutz-Kelker bias due to sample truncation, as stars with negative parallax can be included (though biases due to the earlier sample selection will remain; see Sect. 3.1). For example, assuming that the stars follow a relation between period and absolute magnitude of the form

$M=\alpha \log P+\rho$,

where $P$ is the period and $\alpha$ and $\rho$ are the slope and zero point of the $P L$ and $P W$ relations, we fit

$10^{0.2(\alpha \log P+\rho)}=\varpi 10^{0.2 m_{0}-2}$

using the weighted non-linear least squares. The same approach could be used to fit the $P L Z$ and $M_{V}-[\mathrm{Fe} / \mathrm{H}]$ relations of RR Lyrae stars. We just apply the non-linear least-squares fit to the equations

$10^{0.2(\alpha \log \mathrm{P}+\beta[\mathrm{Fe} / \mathrm{H}]+\rho)}=\varpi 10^{0.2 m_{0}-2}$

and

$10^{0.2(\alpha[\mathrm{Fe} / \mathrm{H}]+\rho)}=\varpi 10^{0.2 m_{0}-2}$.

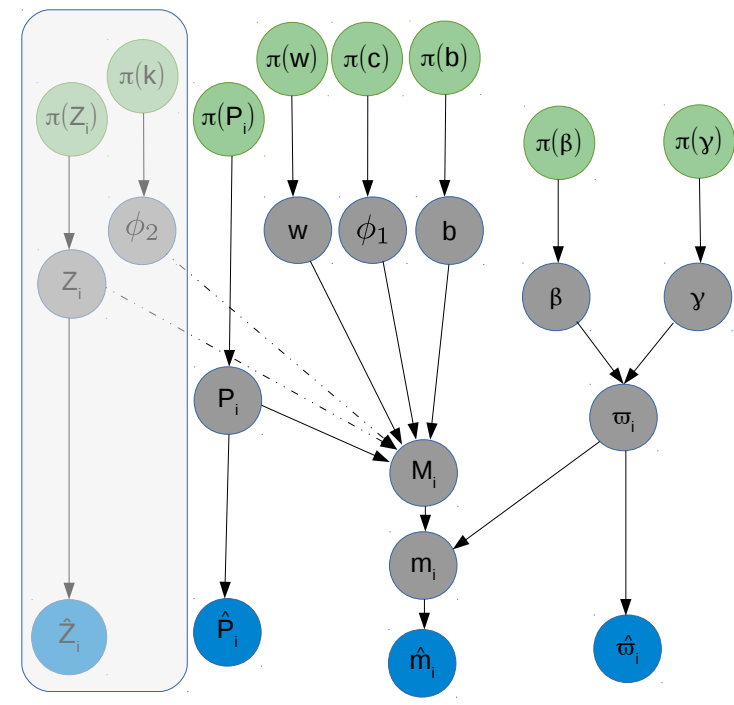

Fig. 19. Directed acyclic graph representing the forward model used to infer the $P L(Z)$ relation coefficients.

Finally, using a Bayesian approach to fit the $P L, P W, P L Z$, or $M_{V}-[\mathrm{Fe} / \mathrm{H}]$ relations followed by the variable stars provides an excellent alternative option. The Bayesian estimate of the $P L(Z)$ relationships is accomplished by means of a hierarchical model (which will be described elsewhere, but for a similar approach see Sesar et al. 2016) encoded in the directed acyclic graph shown in Fig. 19. It shows the measurements at the bottom level: periods $\left(\hat{P}_{i}\right)$, apparent magnitudes $\left(\hat{m}_{i}\right)$, metallicities (when appropriate, $\hat{Z}_{i}$ ), and parallaxes $\left(\hat{\varpi}_{i}\right)$. The subindex $i$ runs from 1 to the total number of stars $N$ in each sample. Our model assumes that the measurements (denoted $\left\{\hat{d}_{i}=\left(\hat{m}_{i}, \hat{P}_{i}, \hat{\varpi}_{i}, \hat{Z}_{i}\right) ; i\right.$ : $1,2, \ldots, N\})$ are realisations from normal distributions centred at the true (unknown) values (denoted $P_{i}, Z_{i}, m_{i}$, and $\varpi_{i}$ ) and with standard deviations given by the measurement uncertainties. We express the most general $P L Z$ relation as

$M=b+c \cdot \log (P)+k \cdot Z+\epsilon$,

where $\epsilon$ is a Gaussian distributed random variable

$\epsilon \sim \mathcal{N}(0, w)$,

which represents the intrinsic dispersion of the relation (not due to measurement uncertainties).

The parallaxes are assumed to be drawn from a log-normal prior of parameters $\beta$ and $\gamma$. The model is hierarchical in the sense that this prior is inferred as part of the model, and $\beta$ and $\gamma$ are themselves prescribed by their own priors (see Table 2 for a full list of priors). We have tried several prior definitions and the results are insensitive to the prior choice except for unreasonable set-ups.

In the following analysis we apply all three methods: (i) the direct transformation of the parallaxes to absolute magnitudes (Eq. (1)) and the weighted linear least-squares fitting (LSQ) in the period-absolute magnitude plane ( $P L, P W, P L Z$ relations) and the absolute magnitude-metallicity plane $\left(M_{V}-[\mathrm{Fe} / \mathrm{H}]\right.$ relation); (ii) the use of ABL to perform the non-linear weighted least-squares fit of Eqs. (4)-(6); and (iii) the realisation of the Bayesian fitting approach. The Bayesian solution corresponds to the maximum a posteriori (MAP) values, and the rms represents the dispersion of the unweighted residuals with respect to this MAP solution. The ABL and Bayesian methods are preferred to 
Table 2. Prior $(\pi)$ definitions for the hierarchical Bayesian model of the $P L(Z)$ relations.

\begin{aligned} & \hline \hline$p\left(m_{i} \mid \varpi_{i}, \phi_{1}, b, \phi_{2}, w, P_{i}, Z_{i}\right) \sim \mathcal{N}\left(m_{i} \mid\right.\left.\tan \left(\phi_{1}\right) \cdot \log _{10}\left(P_{i}\right)+\tan \left(\phi_{2}\right) \cdot Z_{i}+b-5 \cdot \log _{10}\left(\varpi_{i}\right)+10, w\right) \\ & p\left(\varpi_{i} \mid \beta, \gamma\right) \sim \ln \mathcal{N}\left(\varpi_{i} \mid \beta, \gamma\right) \\ & \pi(\gamma) \sim \exp (1) \\ & p(\beta) \sim \mathcal{N}(0,2) \\ & \pi\left(\phi_{1}\right) \sim \mathcal{N}(0,3.14 / 2) \\ & \pi\left(\phi_{2}\right) \sim \mathcal{N}(0,3.14 / 2) \\ & \pi(b) \sim \mathcal{N}(0,10) \\ & \pi(w) \sim \exp (10) \\ & \pi\left(Z_{i}\right) \sim \mathcal{N}\left(Z_{i} \mid 0,5\right) \\ & \pi\left(P_{i}\right) \sim \mathcal{N}\left(P_{i} \mid 0,3\right) \\ &$\hline\end{aligned}

Notes. The symbol $\sim$ should be read as "is distributed according to". The exp expression should not be interpreted as the exponential analytical function, but as the exponential probability density distribution. We use the $\pi$ symbol to refer to the prior probability, and use 3.14 to refer to the half-length of the circle.

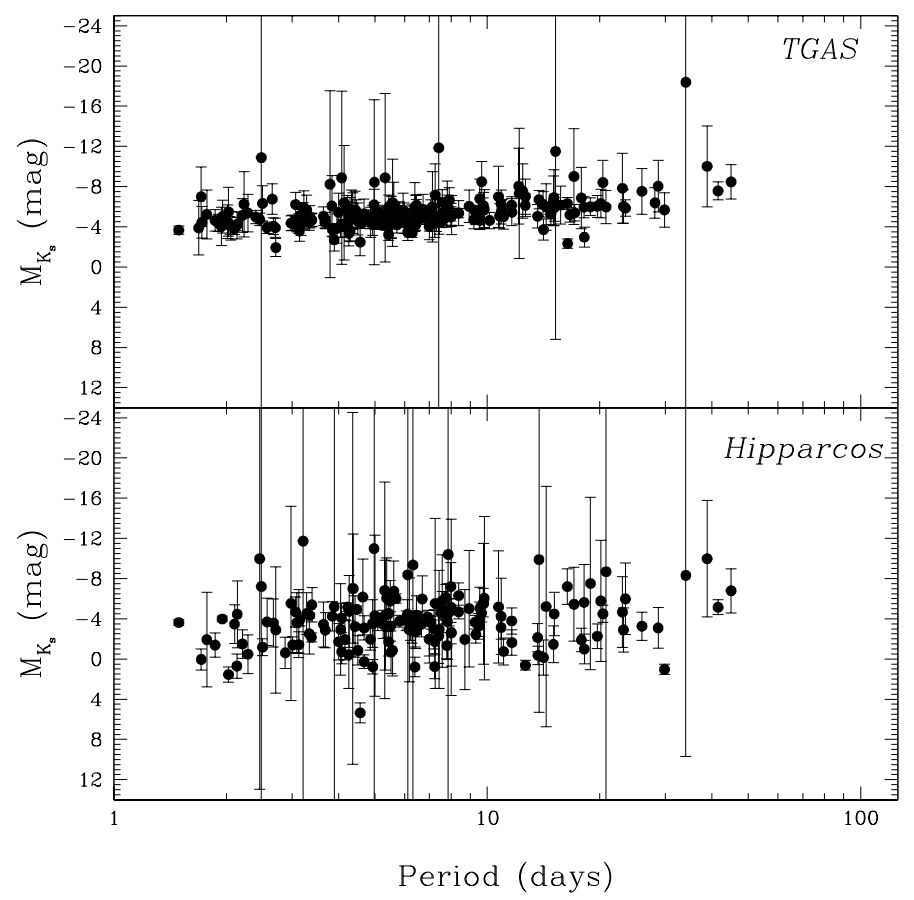

Fig. 20. Classical Cepheid $P M_{K_{\mathrm{S}}}$ relations using TGAS (upper panel) and HIPPARCOS (lower panel) parallaxes. The upper panel shows the absolute magnitudes, $M_{K_{\mathrm{s}}}$, of 221 classical Cepheids with positive parallaxes from TGAS. The lower panel shows 156 classical Cepheids with positive HIPPARCos parallaxes.

avoid biases, and we include the LSQ fitting for comparison purposes and also to allow comparison with published results using this method.

\section{Classical Cepheids}

TGAS parallaxes are available in Gaia DR1 for 331 Galactic classical Cepheids. We have collected from the literature $V, I$, and 2MASS $K_{\mathrm{S}}$ photometry; $E(B-V)$ reddening values; and period and classification for the whole sample, making an effort to assemble a catalogue which is as uniform and homogeneous as possible. For the mean magnitudes, we collected intensityaveraged mean $V, I, K_{\mathrm{s}}$ magnitudes based on a complete sampling of the light curves whenever available; in several cases we computed the intensity-averaged mean values ourselves from the light curves published in the literature. Period and $V, I, K_{\mathrm{s}}$ photometry values were taken from different sources, primarily
Groenewegen (1999), Berdnikov et al. (2000), Fernie et al. (1995, DDO Database of Galactic Classical Cepheids), Ngeow (2012), the GCVS (Samus et al. 2007-2015), and the ASAS3 catalogue (Pojmanski 2002), among others. The $E(B-V)$ reddening values and related errors were taken from Fernie et al. (1995), Groenewegen (1999), Turner et al. (2001), Fouqué et al. (2007), and Pejcha \& Kochanek (2012). In a few cases we specifically estimated the reddening for this study from the available photometry. Metal abundances were mainly taken from Genovali et al. (2014), and for a few stars from Ngeow (2012). Information about duplicity is from Klagyivik \& Szabados (2009) and Anderson et al. (2016).

In the literature we found period values for 312 classical Cepheids in our sample $(94 \%) ; E(B-V)$ reddening values for 276 stars $(83 \%)$; and photometry in the $V, I$, and $K_{\mathrm{s}}$ bands for $297(90 \%), 250$ (76\%), and $292(88 \%)$ classical Cepheids, respectively. We provide the complete dataset of the 331 Galactic classical Cepheids in Table A.1. HIPPARCos parallaxes are available for 248 of them. Among the 248 classical Cepheids with parallax measured by both HIPPARCos and Gaia we selected 228 with $K_{\mathrm{s}}$ magnitude, reddening, and period available in the literature. To correct the $K_{\mathrm{s}}$ magnitudes for extinction we used the extinction law of Cardelli et al. (1989) and adopted for the ratio of total to selective extinction the value $R_{\mathrm{V}}=3.1$, thus deriving $K_{\mathrm{s}, 0}=K_{\mathrm{s}}-0.35 E(B-V)$. We then transformed the TGAS and HIPPARCos parallaxes to absolute $M_{K_{\mathrm{s}}}$ magnitudes applying Eq. (1). This transformation was possible only for stars with positive parallax values, namely 221 out of 228 stars with TGAS parallax and only 156 out of 228 for HIPPARCOS. The corresponding $P M_{K_{\mathrm{S}}}$ relations are shown in the upper and lower panels of Fig. 20 for the TGAS and HIPPARCOS parallaxes, respectively. The improvement produced by the TGAS parallaxes is impressive. The scatter is very much reduced, the sample is about $30 \%$ larger, and although the error bars are still very large a clear $P M_{K_{\mathrm{s}}}$ relation can now be seen in the data. Since other factors (e.g. multiplicity, see Sect. 4.1) in addition to errors in the parallax measurements may contribute to the large dispersion seen in the upper panel of Fig. 20, we cleaned the sample from binary systems and retained for analysis only single, fundamental mode, classical Cepheids.

\subsection{Binarity-mutiplicity among Cepheids}

Classical Cepheids are Population I stars; therefore, occurrence of binaries among them is a common phenomenon. However, it is not easy to detect the presence of a companion because it is the 
supergiant Cepheid that dominates the system. For the brightest (naked-eye) Cepheids, the frequency of binaries (including systems consisting of more than two stars) exceeds $50 \%$, and there is a selection effect towards fainter visual magnitudes that results in a deficit in known binaries (Szabados 2003). For classical Cepheids in the MW, there is an online database developed and maintained at the Konkoly Observatory ${ }^{7}$ which contains the actual list of known binaries involving a Cepheid component. A few further binary Cepheids are known from Anderson et al. (2016). When performing astrometric reduction of the Gaia data, these stars have to be treated as binary systems because the orbital motion (if not taken into account) falsifies the resulting trigonometric parallax (Szabados 1997). Moreover, the brightness of the Cepheid ( $G, G_{B P}$, and $G_{R P}$ magnitudes) has to be corrected for the photometric contribution of the companion star. If the available photometric, spectroscopic, and astrometric data are insufficient to characterise the binary system or to get reliable apparent and absolute magnitudes of the Cepheid component, that particular Cepheid cannot be used as a calibrator in determining the zero point of the $P L$ relationship. Combining information from the online database of the Konkoly Observatory, Klagyivik \& Szabados (2009), and Anderson et al. (2016) we find that $198(60 \%)$ of the 331 classical Cepheids in our sample are binaries. They were not used to compute the $P L$ relations. Once known binaries are discarded our sample reduces to 133 non-binary classical Cepheids. The selection for binarity we have operated may sound very strict; however, the reduced scatter of the comparison with photometric parallaxes obtained with the Baade-Wesselink technique (see Fig. 15) when binary Cepheids are discarded indicates that ours is a safe approach.

\subsection{Derivation of the classical Cepheid PL and PW relations}

We found information on the period; $V, I_{\mathrm{c}}, K$ photometry; and reddening only for 125 of the 133 non-binary classical Cepheids. Furthermore, three of them (BB Her, EV Aql, and V733 Aql) are classified as Type II Cepheids in the McMaster Cepheid Photometry and Radial Velocity Data Archive ${ }^{8}$. We discarded them from the sample of classical Cepheids, but did not include them in the list of Type II Cepheids since their classification is uncertain. After this additional cleaning we were left with a sample of 122 bona fide classical Cepheids.

Fundamental mode (F) and first-overtone (FO) classical Cepheids follow different $P L$ relations. Information on the pulsation mode of the 122 classical Cepheids in the clean sample is available from the studies by Udalski et al. (1999), Sziládi et al. (2007), Klagyivik \& Szabados (2009), Molnár \& Szabados (2014), and Inno et al. (2015), which show that our sample contains 102 fundamental mode classical Cepheids. In order to remove any scatter caused by mixing multiple $P L$ relations and given the small number of FO pulsators (20), in the following analysis we consider only the F classical Cepheids. The error distribution of the TGAS parallaxes for the $102 \mathrm{~F}$ classical Cepheids is shown by the magenta histogram in Fig. 1. Relevant information (Gaia/HIPPARCOS/Tycho-2 IDs; $G,\langle V\rangle,\langle I\rangle,\left\langle K_{\mathrm{s}}\right\rangle$ magnitudes; period; reddening; metallicity; parallax; and parallax errors) for them is summarised in the first 102 rows of Table A.1. Typical errors on the $V, I, K_{\mathrm{s}}$ apparent mean magnitudes are estimated to be of about $0.02 \mathrm{mag}$.

We note that $G$-band magnitudes are available for all the classical Cepheids in our sample; however, we decided not

\footnotetext{
http://www. konkoly.hu/CEP/intro.html

8 http://crocus.physics.mcmaster.ca/Cepheid/
}

to compute $G$-band PLs primarily because the Gaia $G$-band throughput is too large $(330-1050 \mathrm{~nm})$, encompassing roughly from the $U$ to the $Y$ spectral ranges. The intrinsic width of the classical Cepheids instability strip varies significantly going from $U$ to $K$ passbands and the dispersion of the Cepheids $P L$ varies accordingly, being tightest in the NIR (Sect. 1 and, e.g. Fig. 4 in Madore \& Freedman 1991), which makes the $K$ band the best choice for testing TGAS parallaxes with the classical Cepheid $P L$ relations. Furthermore, the $G$-band magnitudes available for TGAS classical Cepheids in Gaia DR1 are often the straight average of only a few measurements unevenly sampling the cyclic light variation of these stars, hence further enhancing the scatter of the $G$-band $P L$. The situation will improve significantly with Gaia DR2 because better sampled $G$-band light curves will be released for the TGAS Cepheids and, more importantly, because $G_{R P}$ magnitudes $(640-1050 \mathrm{~nm})$ will become available, allowing the Gaia $G_{R P} P L$ relation to be built for classical Cepheids.

We computed $P L$ relations in the $V, I$, and $K_{\mathrm{S}}$ bands (hereafter $\left.P M_{V}, P M_{I}, P M_{K_{\mathrm{s}}}\right)$, as well as $(V, V-I),\left(K_{\mathrm{s}}, V-K_{\mathrm{s}}\right) P W$ relations (hereafter $\left.P W(V, V-I), P W\left(K_{\mathrm{s}}, V-K_{\mathrm{s}}\right)\right)$ for the 102 $F$ classical Cepheids in our sample. To correct for extinction the $V, I$, and $K_{\mathrm{s}}$ magnitudes we adopted the extinction relations from Cardelli et al. (1989) and $R_{V}=3.1$, thus obtaining $V_{0}=V-3.1 E(B-V), I_{0}=I-1.48 E(B-V)$, and $K_{\mathrm{s}, 0}=$ $K_{\mathrm{s}}-0.35 E(B-V)$. The Wesenheit magnitudes used in this paper are defined as follows: $W(V, I)=V-2.55(V-I)$ (Fouqué et al. 2007) and $W\left(V, K_{\mathrm{s}}\right)=K_{\mathrm{s}}-0.13\left(V-K_{\mathrm{s}}\right)$ (Ripepi et al. 2012). As pointed out in Sect. 1, the $P W$ relations have several advantages with respect to normal $P L$ relations: the effect of errors on the reddening estimates is in principle removed, in practice greatly mitigated, and the colour term in the $W$ magnitude definition takes into account and partially corrects for the finite colour width of the instability strip, thus reducing the associated uncertainty on the distance determinations. It is also worth noticing that in the case of the $W\left(V, K_{\mathrm{s}}\right)$, the $P W$ is equivalent to the $P L C$ relation in the same filters (see e.g. Ripepi et al. 2012). Since errors on the TGAS parallaxes are large we did not attempt to derive both slope and zero point, but fixed the slope of the $P L$ and $P W$ relations and used the TGAS parallaxes just to estimate the zero points. We adopted the slopes from Fouqué et al. (2007) for the $P M_{V}, P M_{I}, P M_{K_{\mathrm{s}}}$, and $P W(V, V-I)$ relations and from Ripepi et al. (2012) for the $P W\left(K_{\mathrm{s}}, V-K_{\mathrm{s}}\right)$ relation.

The $P L$ and $P W$ relations obtained by applying the three different approaches described in Sect. 3.2 to the various passbands considered in this paper $\left(V, I\right.$ and $\left.K_{\mathrm{s}}\right)$ are listed in Table 3 and shown in Fig. 21 for the case of the $P K_{\mathrm{s}}$ relations. Specifically, the upper panel of Fig. 21 shows the LSQ of the absolute $M_{K_{\mathrm{s}}}$ magnitudes obtained by direct transformation of the parallaxes (Eq. (1)). The fit was possible only for 95 classical Cepheids in the sample for which TGAS parallaxes have positive values. The $P M_{V}, P M_{I}, P M_{K_{\mathrm{s}}}, P W(V, V-I)$, and $P W\left(K_{\mathrm{s}}, V-K_{\mathrm{s}}\right)$ relations were then computed using the ABL method and a weighted nonlinear least-squares fit in the form of Eq. (4). Using the ABL approach, it is possible to use the whole sample of 102 classical Cepheids without discarding stars with negative parallaxes. The $P M_{K_{\mathrm{s}}}$ relation derived with the ABL method is shown in the middle panel of Fig. 21. Finally, we used our Bayesian approach to fit the $P L$ and $P W$ relations of the whole sample of 102 classical Cepheids, the resulting $P M_{K_{\mathrm{s}}}$ relation is presented in the bottom panel of Fig. 21. Comparison of the results in Table 3 shows that the ABL and Bayesian approaches are generally in good agreement with each other and provide brighter absolute magnitudes (hence longer distances) than the direct transformation of 
Table 3. $P L$ and $P W$ relations for classical Cepheids with zero point based on TGAS parallaxes.

\begin{tabular}{lcc}
\hline \hline & Relation & $\begin{array}{c}\text { rms } \\
(\mathrm{mag})\end{array}$ \\
\hline$P M_{V}$ 95 objects (LSQ) & $-2.678 \log P-(1.00 \pm 0.08)$ & 0.74 \\
$P M_{V} 102$ objects (ABL) & $-2.678 \log P-(1.54 \pm 0.10)$ & 0.85 \\
$P M_{V} 102$ objects (BA) & $-2.678 \log P-\left(1.49_{-0.11}^{+0.12}\right)$ & 1.31 \\
\hline$P M_{I} 95$ objects (LSQ) & $-2.98 \log P-(1.28 \pm 0.08)$ & 0.78 \\
$P M_{I} 102$ objects (ABL) & $-2.98 \log P-(1.84 \pm 0.10)$ & 0.87 \\
$P M_{I} 102$ stars (BA) & $-2.98 \log P-\left(1.80_{-0.12}^{+0.13}\right)$ & 1.33 \\
\hline$P M_{\mathrm{K}_{\mathrm{s}}} 95$ objects $(\mathrm{LSQ})$ & $-3.365 \log P-(2.06 \pm 0.08)$ & 0.74 \\
$P M_{\mathrm{K}} 102$ objects $(\mathrm{ABL})$ & $-3.365 \log P-(2.63 \pm 0.10)$ & 0.88 \\
$P M_{K_{S}} 102$ stars $(\mathrm{BA})$ & $-3.365 \log P-\left(2.60_{-0.15}^{+0.11}\right)$ & 1.33 \\
\hline$P W(V, V-I) 95$ objects (LSQ) & $-3.477 \log P-(2.21 \pm 0.08)$ & 0.77 \\
$P W(V, V-I) 102$ objects (ABL) & $-3.477 \log P-(2.82 \pm 0.11)$ & 0.90 \\
$P W(V, V-I) 102$ stars (BA) & $-3.477 \log P-(2.63 \pm 0.13)$ & 1.36 \\
\hline$P W\left(K_{\mathrm{s}}, V-K_{\mathrm{s}}\right) 95$ objects (LSQ) & $-3.32 \log P-(2.32 \pm 0.08)$ & 0.73 \\
$P W\left(K_{\mathrm{s}}, V-K_{\mathrm{s}}\right) 102$ objects (ABL) & $-3.32 \log P-(2.87 \pm 0.10)$ & 0.87 \\
$P W\left(K_{\mathrm{s}}, V-K_{\mathrm{s}}\right) 102$ stars (BA) & $-3.32 \log P-\left(2.81_{-0.12}^{+0.14}\right)$ & 1.33 \\
\hline
\end{tabular}

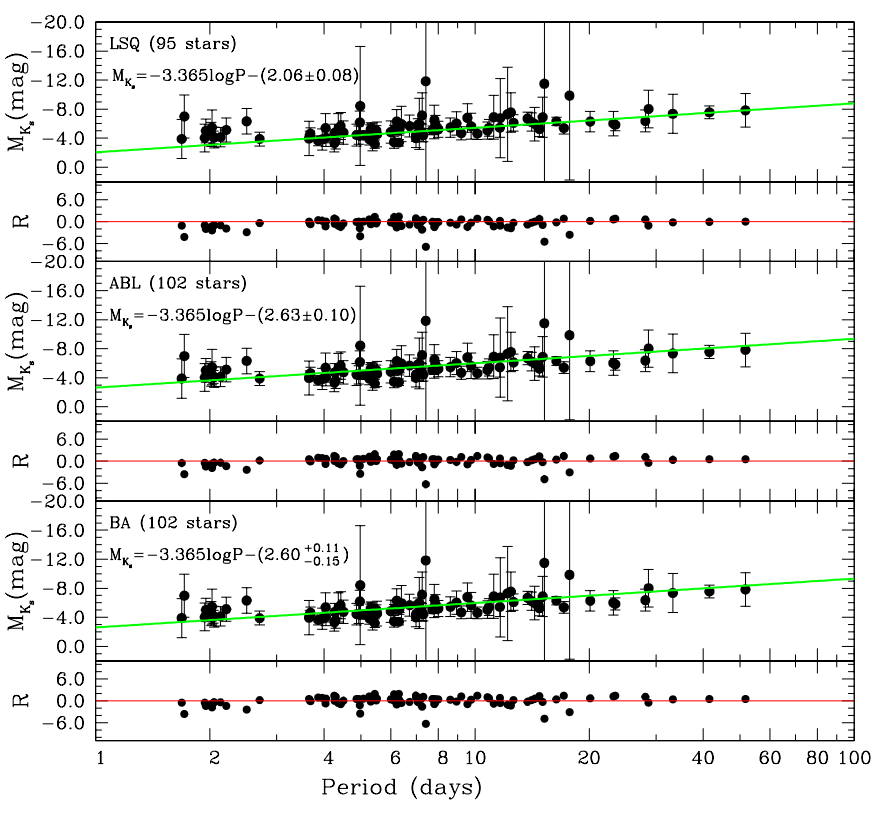

Fig. 21. Classical Cepheid $P L$ relations in the $K_{\mathrm{s}}$-band obtained i) by linear least-squares fitting the stars' absolute magnitudes inferred from direct transformation of the TGAS parallaxes (upper panel); ii) via nonlinear least-squares fit and the ABL method (middle panel); and iii) using a Bayesian approach (bottom panel). The slope of the fit is adopted from Fouqué et al. (2007). The bottom part of each panel shows the residuals from the best fit line.

parallaxes and the LSQ fit whose absolute magnitudes are about $\sim 0.5$ mag systematically fainter. However, we also note that the rms scatter of all relations is very large, due to the large parallax uncertainties. The rms of the Bayesian solution is particularly large as it represents the unweighted residuals with respect to the maximum a posteriori solution.

\section{Type II Cepheids}

Type II Cepheids are pulsating variables that belong to the Population II star family. They have been studied by several authors (Wallerstein \& Cox 1984; Gingold 1985; Harris 1985; Bono et al. 1997; Wallerstein 2002; Feast et al. 2008; Matsunaga et al. 2011; Soszyński et al. 2008; Ripepi et al. 2015) and are usually divided into three classes: BL Herculis (BL Her) with periods from 1 to 4 days, W Virginis (W Vir) with periods from 4 to 20 days, and RV Tauri (RV Tau) with periods from 20 to 150 days. The light curves of BL Her and W Vir stars can be almost sinusoidal or highly non-sinusoidal, those of RV Tauri have alternating minima. It is believed that BL Her variables are low-mass stars $\left(0.5-0.6 M_{\odot}\right)$ that start the central He burning on the blue side of the RR Lyrae star gap and cross the instability strip at about $0.5-1.5$ mag brighter than the RR Lyrae stars (hence the longer periods). Hydrodynamic models indicate that they pulsate in the fundamental radial mode (see e.g. Marconi \& Di Criscienzo 2007, and references therein). The W Vir variables, instead, cross the instability strip during their blue-loop excursions ("blue-nose") from the asymptotic giant branch (AGB) during helium-shell flashes. They are 2-4 mag brighter than the RR Lyrae stars. Like the BL Her stars, the W Vir stars are also thought to pulsate in the radial fundamental mode (see e.g. Lemasle et al. 2015, and references therein).

The RV Tau are post-AGB stars that cross the instability strip at high luminosity during their path to the white dwarf cooling sequence. It is still unclear whether it is appropriate to include them in the same class as the BL Her and W Vir stars since they have different evolutionary histories (Wallerstein 2002). In addition to the three types listed above, Soszyński et al. (2008) suggested the existence of peculiar W Vir (pW Vir) stars. They exhibit peculiar light curves and, at constant period, are generally brighter than normal Type II Cepheids. Although their true nature remains uncertain, it is likely that the $\mathrm{pW}$ Vir are part of binary systems.

It has been known for a long time that the Type II Cepheids follow a PL relation (Nemec et al. 1994) in the optical. Kubiak \& Udalski (2003) later found that all Type II Cepheids with periods in the range from 0.7 to about 10 days in the OGLE II sample (Udalski et al. 1992) follow the same $P L$ relation as then also confirmed by Pritzl et al. (2003) and Matsunaga et al. (2006) for Type II Cepheids in Galactic globular clusters, by Groenewegen et al. (2008) for Type II Cepheids in the Galactic bulge, and on the basis of OGLE III data by 
Table 4. $P L$ and $P W$ relations for Type II Cepheids based on TGAS parallaxes.

\begin{tabular}{lcc}
\hline \hline & Relation & $\begin{array}{c}\text { rms } \\
(\mathrm{mag})\end{array}$ \\
\hline$P M_{J} 22$ objects (LSQ) & $-2.19 \log P-(0.97 \pm 0.13)$ & 0.88 \\
$P M_{J} 26$ objects (ABL) & $-2.19 \log P-(1.50 \pm 0.20)$ & 1.25 \\
$P M_{J} 26$ objects (BA) & $-2.19 \log P-\left(1.36_{-0.25}^{+0.26}\right)$ & 1.15 \\
\hline$P M_{K_{\mathrm{s}}} 22$ objects (LSQ) & $-2.385 \log P-(1.18 \pm 0.12)$ & 0.81 \\
$P M_{K_{\mathrm{s}}} 26$ objects (ABL) & $-2.385 \log P-(1.58 \pm 0.17)$ & 1.10 \\
$P M_{K_{\mathrm{s}}} 26$ objects (BA) & $-2.385 \log P-\left(1.51_{-0.22}^{+0.23}\right)$ & 1.14 \\
\hline$P W\left(K_{\mathrm{s}}, J-K_{\mathrm{s}}\right) 22$ objects $(\mathrm{LSQ})$ & $-2.52 \log P-(1.34 \pm 0.10)$ & 0.80 \\
$P W\left(K_{\mathrm{s}}, J-K_{\mathrm{s}}\right) 26$ objects $(\mathrm{ABL})$ & $-2.52 \log P-(1.59 \pm 0.13)$ & 1.04 \\
$P W\left(K_{\mathrm{s}}, J-K_{\mathrm{s}}\right) 26$ objects $(\mathrm{BA})$ & $-2.52 \log P-\left(1.66_{-0.22}^{+0.21}\right)$ & 1.13 \\
\hline
\end{tabular}

Soszyński et al. (2008) for the LMC sample. Matsunaga et al. (2011) using single-epoch data showed that the Type II Cepheids follow tight $P L$ relations in the $J, H, K_{\mathrm{s}}$ passbands. This result has been confirmed by Ripepi et al. (2015) based on multi-epoch $J, K_{\mathrm{s}}$ photometry for 130 Type II Cepheids observed in the LMC by the VISTA Survey of the Magellanic Clouds system (VMC; Cioni et al. 2011). These authors found that unique $P L$ and $P W$ relations hold both for BL Her and W Vir variables, whereas RV Tau stars follow different and more dispersed relationships. In light of this and the different evolutionary history, we have not considered the RV Tau stars in our analysis. Ripepi et al. (2015) also found that the metallicity dependence of the Type II Cepheid $P L$ and $P W$ relations, if present, is small. Therefore, the metallicity was neglected in our analysis.

The sample of 31 Type II Cepheids that have TGAS parallax (see Sect. 2) contains 12 BL Her, 14 W Vir, and 5 RV Tau stars. Excluding the RV Tau variables we are left with a sample of 26 stars spanning the period range from 1.16 to $30.0 \mathrm{~d}$. The error distribution of the TGAS parallaxes for these 26 Type II Cepheids is shown by the grey histogram in Fig. 2. Their $J$ and $K_{\mathrm{s}}$ photometry was taken from the 2MASS catalogue (Cutri et al. 2003); the pulsation periods were taken from the GCVS (Samus et al. 2007-2015); and the $E(B-V)$ reddening values and related errors were taken from the NASA/IPAC Infrared Science Archive ${ }^{9}$, which is based on the reddening maps of Schlafly \& Finkbeiner (2011). We provide the complete dataset of the 31 Type II Cepheids in Table A.2. To de-redden the $K_{\mathrm{s}}$ and $J$ magnitudes we applied the extinction laws $A_{J}=$ $0.87 E(B-V)$ and $A_{K}=0.35 E(B-V)$ from Cardelli et al. (1989), adopting $R_{V}=3.1$. We also calculated the Wesenheit magnitude $W\left(K_{\mathrm{s}}, J\right)=K_{\mathrm{s}}-0.69\left(J-K_{\mathrm{s}}\right)$ and fitted the $P M_{K_{\mathrm{s}}}, P M_{J}$, and $P W\left(K_{\mathrm{s}}, J-K_{\mathrm{s}}\right)$ relations, adopting the slopes derived by Ripepi et al. (2015) for BL Her and W Wir stars in the LMC. For the Type II Cepheids we only have single-phase 2MASS $J$ and $K_{\mathrm{s}}$ magnitudes instead of magnitudes averaged over the whole pulsation cycle, which introduced additional uncertainty. The typical amplitude of the Type II Cepheids in these bands is $\sim 0.3$ mag, and so we adopted mean errors of the apparent $J$ and $K_{\mathrm{s}}$ magnitudes of $0.15 \mathrm{mag}$. The sample of 26 Type II Cepheids contains four stars with negative parallaxes, hence we performed the linear least-squares fitting of the absolute magnitudes only for the 22 Type II Cepheids with a positive parallax, while we applied the ABL and Bayesian methods to the whole sample of 26 Type II Cepheids. As we did for the classical Cepheids, in Fig. 22 we show only the $P M_{K_{\mathrm{s}}}$ relation and summarise in Table 4 the relations obtained in all various bands considered in

9 http://irsa.ipac.caltech.edu/applications/DUST/

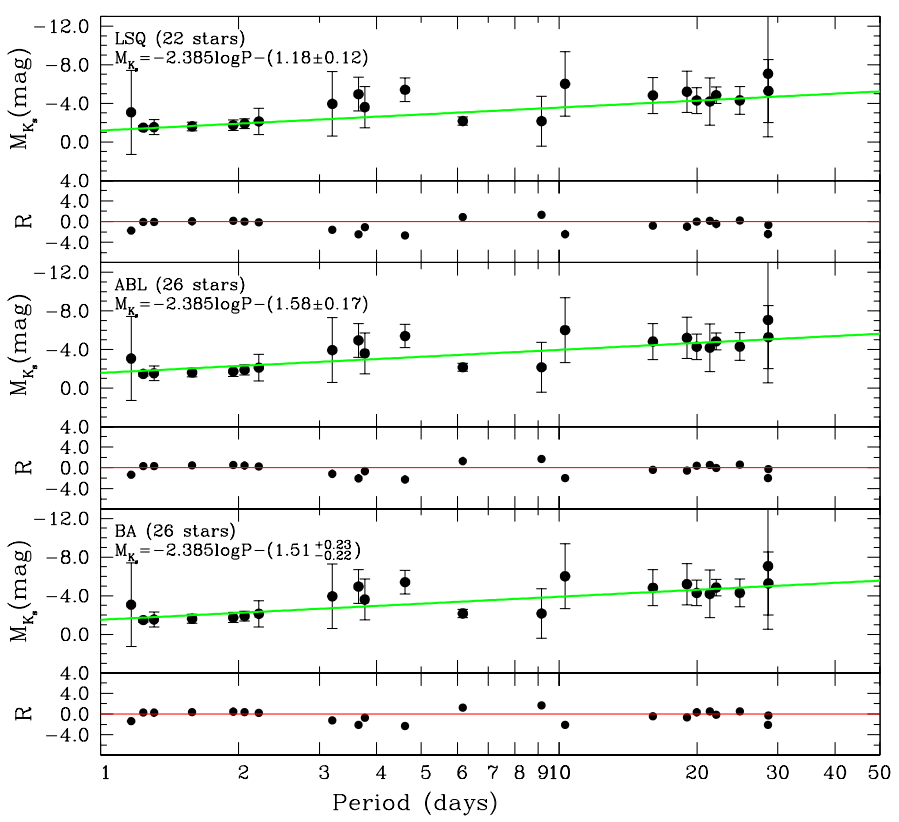

Fig. 22. $P L$ relation in the $K_{\mathrm{s}}$ band of the Type II Cepheids obtained i) via linear least-squares fits of the stars' absolute magnitudes inferred by direct transformation of the TGAS parallaxes (upper panel); ii) via non-linear least-squares fits and the ABL method (middle panel); and iii) using the Bayesian approach (bottom panel). The slope of the fit is adopted from Ripepi et al. (2015). The bottom part of each panel shows the residuals from the best fit line.

the paper. Similarly to what we found for classical Cepheids, the rms of all relations is fairly large; the ABL and Bayesian approaches are generally in good agreement with each other and are, on average, about 0.4 mag brighter than was found with the LSQ fit.

\section{RR Lyrae stars}

TGAS parallaxes are published in Gaia DR1 for 364 MW RR Lyrae stars. Photometry and metallicity for most of these stars is available in the literature, although sparse through many different papers and catalogues. Dambis et al. (2013) have collected and homogenised the literature data of $403 \mathrm{MW}$ RR Lyrae stars for which they publish period, pulsation mode, interstellar visual absorption $\left(A_{V}\right)$, iron abundance $([\mathrm{Fe} / \mathrm{H}])$ on the Zinn \& West (1984) metallicity scale (for 402 stars), and intensity-averaged mean magnitudes in the Johnson $V$, 2MASS $K_{\mathrm{s}}$, and Wide-field Infrared Survey Explorer (WISE) $\mathrm{W}_{1}$ 


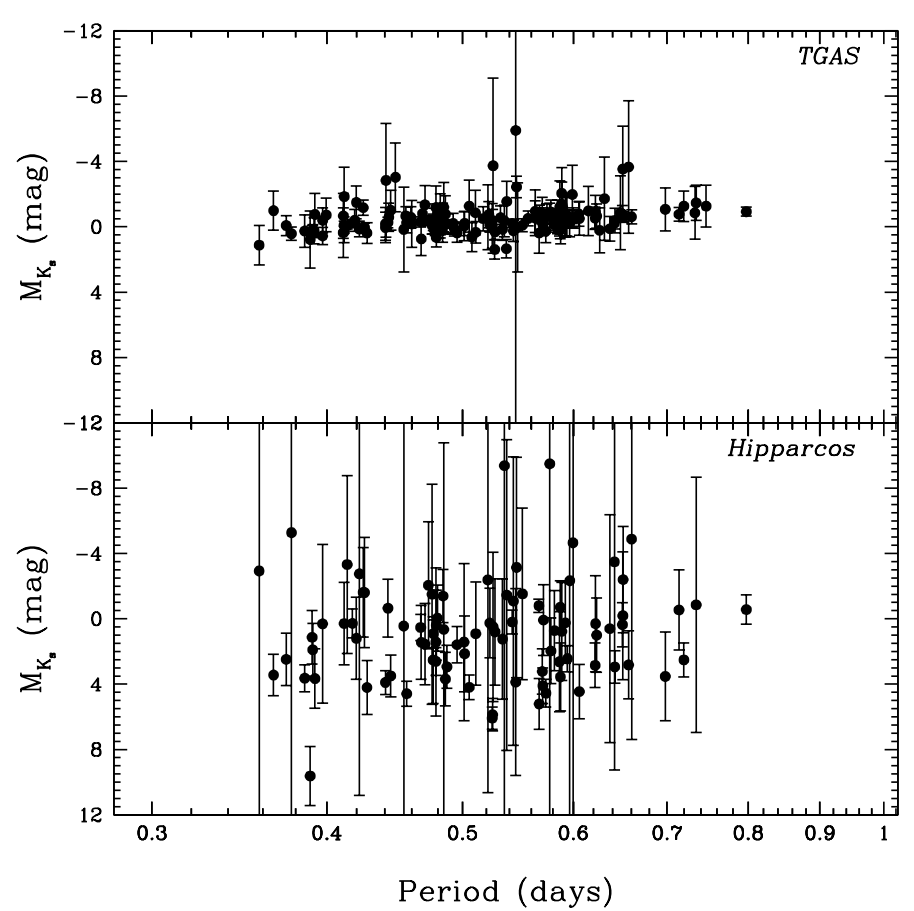

Fig. 23. RR Lyrae star $P M_{K_{\mathrm{s}}}$ relations using TGAS (upper panel) and HIPPARCos (lower panel) parallaxes. The upper panel shows the absolute magnitudes, $M_{K_{\mathrm{s}}}$, of 143 RR Lyrae stars with positive TGAS parallaxes. The lower panel shows $91 \mathrm{RR}$ Lyrae stars with positive HIPPARCos parallaxes.

(3.4 $\mu \mathrm{m})$ passbands for 384, 403, and 398 stars, respectively. The pulsation periods are taken from the ASAS3 (Pojmanski 2002; Maintz 2005) and GCVS (Samus et al. 2007-2015) catalogues. The interstellar extinction values are estimated from the 3D model by Drimmel et al. (2003). These authors do not provide individual errors for the extinction values, but they compared their extinction estimates with those derived from NIR colourmagnitude diagrams of different MW fields based on 2MASS data, finding differences smaller than 0.05 mag. We adopt this value as the mean uncertainty of the extinction. The mean $V$ magnitudes were calculated from nine overlapping sets of observations (see details in Dambis et al. 2013, and references therein); the $K_{\mathrm{s}}$-band mean magnitudes were calculated from the 2MASS single-epoch observations of Cutri et al. (2003) and by applying the phase-correction procedure described in Feast et al. (2008) ${ }^{10}$. Mean magnitudes in the MIR $W 1$ passband were calculated from the WISE single-exposure database. We cross-matched our sample of 364 RR Lyrae stars with TGAS parallaxes against the catalogue of Dambis et al. (2013) and found 200 sources in common. They span the period range from 0.27 to $0.80 \mathrm{~d}$. The error distribution of the TGAS parallaxes for these 200 RR Lyrae stars is shown by the blue histogram in Fig. 3. The complete dataset (Gaia/HIPPARCOS/Tycho-2 IDs; period; pulsation mode; $G,\langle V\rangle,\left\langle K_{\mathrm{s}}\right\rangle,\left\langle W_{1}\right\rangle$ magnitudes; $A_{V}$ and $[\mathrm{Fe} / \mathrm{H}]$ values) along with TGAS parallaxes and errors for the total sample of 364 RR Lyrae stars is presented in Table A.3. For the first 200 entries in the table, the literature values are from Dambis et al. (2013), for the remaining 164 sources the literature information is mainly taken from the GCVS (Samus et al. 2007-2015).

\footnotetext{
${ }^{10}$ For 32 RR Lyrae stars in the Dambis et al. (2013) sample, the 2MASS magnitudes do not have phase correction; however, only one of them falls in our sample of 364 stars.
}

Both HIPPARCos and TGAS parallaxes are available for 145 RR Lyrae stars out of 200. As for the classical Cepheids we transformed the TGAS and HIPPARCos parallaxes to absolute $M_{\mathrm{K}_{\mathrm{s}}}$ magnitudes applying Eq. (1). This transformation was possible only for stars with positive parallax values, namely 143 out of 145 stars with TGAS parallax and only 91 out of 145 for HIPPARCOS. We "fundamentalised" the periods of the RRc stars by adding 0.127 to the $\log P$. The corresponding $P M_{\mathrm{K}_{\mathrm{s}}}$ relations are shown in the upper and lower panels of Fig. 23 for TGAS and HIPPARCos parallaxes, respectively. The former shows the significant improvement of the TGAS parallaxes for RR Lyrae stars. This is much more impressive than for classical Cepheids and reveals a $P M_{\mathrm{K}_{\mathrm{s}}}$ relation which becomes markedly visible when compared to the HIPPARCOS values.

Since Dambis et al. (2013) provide a homogeneous dataset for a fairly large sample of RR Lyrae stars, we used their sample to study the $P L$ relations in the $K_{\mathrm{s}}$ and $W 1$ passbands, the $P L Z$ relation in the $K_{\mathrm{s}}$ band, and the optical $M_{V}-[\mathrm{Fe} / \mathrm{H}]$ relation adopting the three different approaches described in Sect. 3.2. The occurence of RR Lyrae stars in binary systems is an extremely rare event. Only one RR Lyrae star, TU UMa, is a confirmed member of a binary star system; it has an orbital period of approximately $23 \mathrm{yr}$ (Wade et al. 1999). Hence, we do not expect extra-scatter in the RR Lyrae relations due to this effect. Indeed, TU UMa is in the sample of 200 RR Lyrae stars that we used to derive the relations for the RR Lyrae stars described in the following sections, and it is found to fall very nicely on the best fit line of the various relations.

We note that we did not use the $G$-band magnitudes to compute the $P L, P L Z$, or the $M_{V}-[\mathrm{Fe} / \mathrm{H}]$ relations of the RR Lyrae stars, for the same reason as discussed for classical Cepheids in Sect. 4.2 and even more so for the RR Lyrae stars. In fact, as clearly shown by Fig. 2 in Catelan et al. (2004), the slope of the RR Lyrae $P L$ relation changes from positive to negative values moving from the blue to the red edges of the Gaia $G$ passband, being roughly zero at its center. Hence, such a large passband should not be used to derive the RR Lyrae $P L$ and $M_{V}-[\mathrm{Fe} / \mathrm{H}]$ relations.

\subsection{Derivation of the $R R$ Lyrae stars $P L$ and $P L Z$ relations in the $K_{s}$ passband}

The NIR $P M_{K} Z$ relation of RR Lyrae stars has been studied by many different authors and, as summarised in Sect. 1, coefficients and zero point of the relation differ significantly; the literature values for the dependence of $M_{\mathrm{K}_{\mathrm{s}}}$ on period range from -2.101 (Bono et al. 2003) to -2.73 (Muraveva et al. 2015) and the dependence on metallicity ranges from 0.03 (Muraveva et al. 2015) to 0.231 (Bono et al. 2003). We used the sample of 200 RR Lyrae stars with TGAS parallaxes along with $K_{\mathrm{s}}$ magnitudes, period, and metallicity values from Dambis et al. (2013) to fit the $P M_{K_{\mathrm{s}}}$ and $P M_{K_{\mathrm{s}}} Z$ relations. The $K_{\mathrm{s}}$ magnitudes were de-reddened using the $V$-band absorption values $\left(A_{V}\right)$ from Dambis et al. (2013), and the $A_{K} / A_{V}=0.114$ extinction law for the $K$ band from Cardelli et al. (1989). The periods of the first overtone RR Lyrae stars (RRc) were fundamentalised as described in the previous section. As with the Cepheids, we did not attempt to derive both slope and zero point of the RR Lyrae relations, but used the TGAS parallaxes only to estimate the zero points. Specifically, we adopted the slope of the $P M_{K_{\mathrm{s}}}$ relation from Muraveva et al. (2015), who studied RR Lyrae stars in the LMC. The upper panel of Fig. 24 shows the linear least-squares fit of the absolute magnitudes inferred from the direct transformation of the TGAS parallaxes. Five RR Lyrae stars in the 
Table 5. $P L$ and $M_{V}-[\mathrm{Fe} / \mathrm{H}]$ relations for RR Lyrae stars based on TGAS parallaxes.

\begin{tabular}{lcc}
\hline \hline & Relation & $\begin{array}{c}\text { rms } \\
(\mathrm{mag})\end{array}$ \\
\hline$P M_{K_{\mathrm{s}}}$ 195 stars (LSQ) & $-2.73 \log P-(1.06 \pm 0.04)$ & 0.84 \\
$P M_{K_{\mathrm{s}}} 200$ stars (ABL) & $-2.73 \log P-(1.26 \pm 0.04)$ & 0.90 \\
$P M_{K_{\mathrm{s}}}$ 200 stars (BA) & $-2.73 \log P-(1.24 \pm 0.05)$ & 1.02 \\
\hline$P M_{K_{\mathrm{s}}} Z 195$ stars (LSQ) & $-2.73 \log P-(0.01 \pm 0.07)[\mathrm{Fe} / \mathrm{H}]-(1.08 \pm 0.09)$ & 0.84 \\
$P M_{K_{\mathrm{s}}} Z$ 200 stars (ABL) & $-2.73 \log P+(0.07 \pm 0.07)[\mathrm{Fe} / \mathrm{H}]-(1.17 \pm 0.10)$ & 0.89 \\
$P M_{K_{\mathrm{s}}} Z 200$ stars (BA) & $-2.73 \log P-\left(0.01_{-0.07}^{+0.11}\right)[\mathrm{Fe} / \mathrm{H}]-\left(1.22_{-0.09}^{+0.40}\right)$ & 1.02 \\
\hline$P M_{W_{1}} 193$ stars (LSQ) & $-2.44 \log P-(1.02 \pm 0.04)$ & 0.82 \\
$P M_{W_{1}} 198$ stars (ABL) & $-2.44 \log P-(1.21 \pm 0.04)$ & 0.87 \\
$P M_{W_{1}} 198$ stars (BA) & $-2.44 \log P-(1.20 \pm 0.05)$ & 1.02 \\
\hline$M_{V}-[\mathrm{Fe} / \mathrm{H}] 195$ stars $(\mathrm{LSQ})$ & $0.214[\mathrm{Fe} / \mathrm{H}]+(1.01 \pm 0.04)$ & 0.82 \\
$M_{V}-[\mathrm{Fe} / \mathrm{H}]$ 200 stars (ABL) & $0.214[\mathrm{Fe} / \mathrm{H}]+(0.82 \pm 0.04)$ & 0.87 \\
$M_{V}-[\mathrm{Fe} / \mathrm{H}] 200$ stars $(\mathrm{BA})$ & $0.214[\mathrm{Fe} / \mathrm{H}]+\left(0.88_{-0.06}^{+0.04}\right)$ & 1.21 \\
\hline
\end{tabular}

sample have a negative parallax value, hence the least-squares fit could be applied only to 195 RR Lyrae stars. The middle and bottom panels of Fig. 24 show the $P M_{K_{\mathrm{s}}}$ relations obtained with the ABL and the Bayesian approaches, respectively. The whole sample of 200 RR Lyrae stars was used with these two methods. The $P M_{K_{\mathrm{s}}}$ relations obtained with the three different approaches are summarised in the first three rows of Table 5. Once again, the rms of all relations is significantly large; the zero points obtained with the ABL and Bayesian approaches are in good agreement with each other and are, on average, about 0.2 mag brighter than the values found with the LSQ fit. We also note that the zero points obtained with the ABL and Bayesian approaches are in perfect agreement with the zero point of the $P M_{K_{\mathrm{s}}} Z$ relation obtained in Muraveva et al. (2015) using the HST parallaxes of four RR Lyrae stars from Benedict et al. (2011) (Eq. (6) in Muraveva et al. 2015).

To fit the RR Lyrae NIR $P M_{K_{\mathrm{s}}} Z$ relation we used the metallicities in Dambis et al. (2013), which are on the Zinn $\&$ West metallicity scale, and took the slope of the period term from Muraveva et al. (2015), who used the metallicity scale defined in Gratton et al. (2004). This is systematically 0.06 dex higher than the Zinn \& West scale, and we transformed the Dambis et al. (2013) metallicities accordingly. Furthermore, Dambis et al. (2013) do not provide errors on the metallicities. We assumed them to be 0.2 dex for all stars, as an average between spectroscopic determinations, whose uncertainties are generally of the order of 0.1 dex, and photometric metallicities, whose typical errors can be as large as 0.3 dex. The $P M_{K_{\mathrm{s}}} Z$ relations obtained via the three different approaches are provided in rows 4 to 6 of Table 5 . The zero points obtained with the ABL and Bayesian approaches are in good agreement with each other and, on average, about 0.1 mag brighter than found with the LSQ fit. The dependence of the $M_{K_{\mathrm{s}}}$ magnitude on metallicity is always found to be negligible considering the current uncertainties.

\subsection{Derivation of the $R R$ Lyrae stars $P L$ relation in the $W_{1}$ passband.}

The MIR $P W_{1}$ relation of RR Lyrae variables has been studied by Madore et al. (2013), Dambis et al. (2014), Klein et al. (2014), and Neeley et al. (2015); the slope of the $P M_{W_{1}}$ dependence

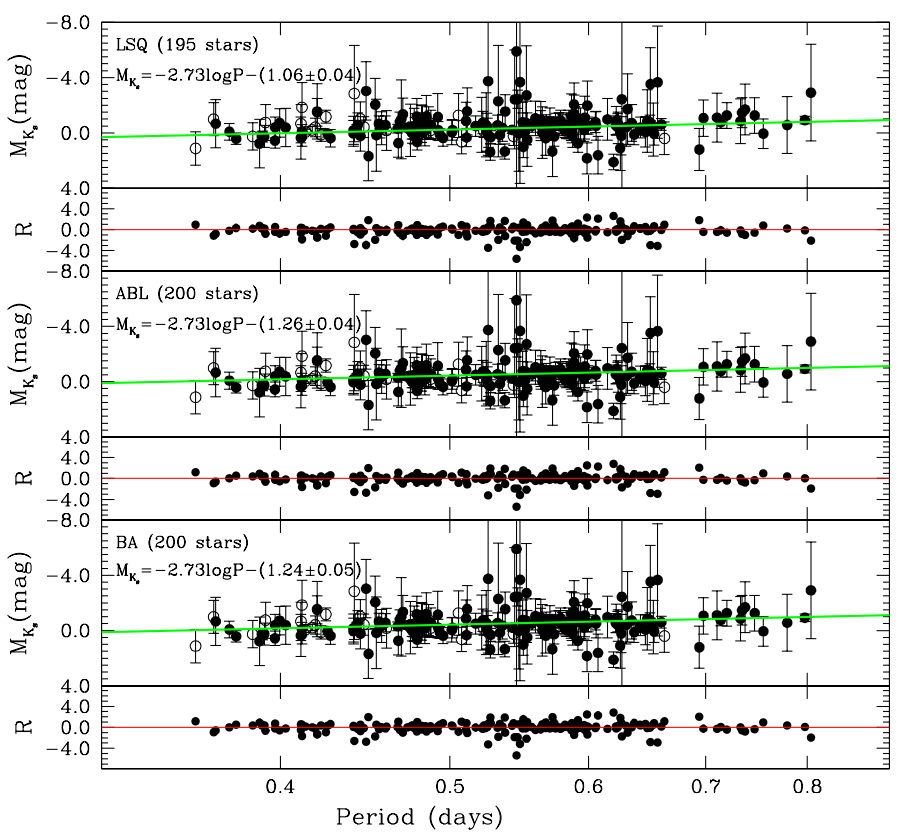

Fig. 24. RR Lyrae $P L$ relation in the $K_{\mathrm{s}}$ obtained i) via linear leastsquares fits of the stars' absolute magnitudes inferred from direct transformation of the TGAS parallaxes (upper panel); ii) via non-linear leastsquares fits and the ABL method (middle panel); and iii) using the Bayesian approach (bottom panel). The slope of the fit is taken from Muraveva et al. (2015). Filled and empty circles represent fundamentalmode (RRab) and first overtone (RRc) stars, respectively. The bottom part of each panel shows the residuals from the best fit line.

on period was found to range from -2.332 (Neeley et al. 2015 for the Spitzer $3.6 \mu \mathrm{m}$ passband) to -2.44 (Madore et al. 2013, for the WISE passbands). The metallicity dependence of the RR Lyrae $P L$ relations is known to decrease with increasing wavelength from NIR to MIR (see Sect. 1). In this paper the dependence on metallicity of the $M_{K_{\mathrm{s}}}$ magnitudes was found to be consistent with zero (see Sect. 6.1); therefore, we do not expect any dependence on metallicity of the $P M_{W_{1}}$ relation to be detectable, given the current large uncertainties. Hence, to compute the $P L$ relation in the $W_{1}$ passband we adopt the slope of the $P M_{W_{1}}$ relation from Madore et al. (2013), who neglect the 
Gaia Collaboration (Clementini, G., et al.): Gaia Data Release 1

Table 6. Distance moduli of the LMC obtained from some of the TGAS-based $P L, P W, P L Z$, and $M_{V}-[\mathrm{Fe} / \mathrm{H}]$ relations derived in this study.

\begin{tabular}{lccc}
\hline \hline Relation & $\begin{array}{c}\mu(\mathrm{LSQ}) \\
(\mathrm{mag})\end{array}$ & $\begin{array}{c}\mu(\mathrm{ABL} \text { method) } \\
(\mathrm{mag})\end{array}$ & $\begin{array}{c}\mu \text { (Bayesian method) } \\
(\mathrm{mag})\end{array}$ \\
\hline \multicolumn{4}{c}{ Classical Cepheids } \\
\hline$P M_{K_{\mathrm{S}}}$ for 95 (102) objects & $18.05 \pm 0.08$ & $18.62 \pm 0.09$ & $18.59 \pm 0.13$ \\
$P W(V, V-I)$ for 95 (102) objects & $18.09 \pm 0.08$ & $18.70 \pm 0.09$ & $18.51 \pm 0.13$ \\
$P W\left(K_{\mathrm{s}}, V-K_{\mathrm{s}}\right)$ for 95 (102) objects & $18.19 \pm 0.07$ & $18.74 \pm 0.09$ & $18.68 \pm 0.13$ \\
\hline \multicolumn{4}{c}{ Type II Cepheids } \\
\hline$P M_{\mathrm{J}}$ for 22 (26) objects & $18.67 \pm 0.19$ & $19.20 \pm 0.24$ & $19.06 \pm 0.22$ \\
$P M_{K_{\mathrm{S}}}$ for 22 (26) objects & $18.65 \pm 0.17$ & $19.05 \pm 0.22$ & $18.98 \pm 0.22$ \\
$P W\left(K_{\mathrm{s}}, J-K_{\mathrm{s}}\right)$ for 22 (26) objects & $18.65 \pm 0.16$ & $19.10 \pm 0.19$ & $18.98 \pm 0.22$ \\
\hline \multicolumn{4}{c}{ RR Lyrae stars } \\
\hline$P M_{K_{\mathrm{s}}}$ for 195 (200) objects & $18.49 \pm 0.06$ & $18.69 \pm 0.06$ & $18.67 \pm 0.07$ \\
$P M_{K_{\mathrm{s}}} Z$ for 195 (200) objects & $18.51 \pm 0.06$ & $18.61 \pm 0.06$ & $18.65 \pm 0.07$ \\
$M_{V}-[\mathrm{Fe} / \mathrm{H}]$ for 195 (200) objects & $18.37 \pm 0.06$ & $18.56 \pm 0.06$ & $18.50 \pm 0.09$ \\
\hline
\end{tabular}

metallicity term. Apparent $W_{1}$ magnitudes are available for 198 of the 200 RR Lyrae stars in our sample (Dambis et al. 2013). Five stars have negative parallaxes, and so when deriving the $P M_{W_{1}}$ relation with the LSQ fit we could use only 193 stars. The ABL and Bayesian approaches were applied instead to the whole sample of 198 stars. To correct for the extinction we used the relation $A_{W_{1}} / A_{V}=0.065$ from Madore et al. (2013). The $P M_{W_{1}}$ relations obtained with the three different approaches are summarised in rows 7 to 9 of Table 5. The zero point of the $P M_{W_{1}}$ derived from the LSQ fit is 0.24 mag fainter than the zero point in Madore et al. (2013) $(-1.26 \pm 0.25)$, which is based on the HST parallaxes of four RR Lyrae stars from Benedict et al. (2011), while the zero points obtained with the $\mathrm{ABL}$ and Bayesian methods are about 0.2 mag brighter and well in agreement, within the errors, with Madore et al. (2013).

\subsection{Derivation of the optical $M_{V}-[\mathrm{Fe} / \mathrm{H}]$ relation of $R R$ Lyrae stars}

We used our sample of 200 RR Lyrae stars to compute the luminosity-metallicity relation, $M_{V}-[\mathrm{Fe} / \mathrm{H}]$, which RR Lyrae stars conform to in the optical. The results are summarised in the bottom three rows of Table 5 for the LSQ fit, ABL method, and Bayesian approach. The direct transformation of the parallaxes to absolute magnitudes was only possible for 195 stars with positive parallaxes, while the ABL and Bayesian approaches were applied to the whole sample of 200 RR Lyrae stars. We corrected the $V$ apparent magnitudes for extinction using $A_{V}=3.1 E(B-V)$ (Cardelli et al. 1989). We adopted the slope of Clementini et al. (2003) and Gratton et al. (2004) who studied the luminosity-metallicity relation of RR Lyrae stars in the LMC. As in Sect. 6.1, we transformed the Dambis et al. (2013) metallicities to the scale adopted in Clementini et al. (2003) and Gratton et al. (2004). The $M_{V}-[\mathrm{Fe} / \mathrm{H}]$ relation derived by the direct transformation of the TGAS parallaxes provides an absolute magnitude of $M_{V}=0.69 \pm 0.04 \mathrm{mag}$ for RR Lyrae stars with metallicity $[\mathrm{Fe} / \mathrm{H}]=-1.5 \mathrm{dex}$. This is significantly fainter than the value of $M_{V}=0.45 \pm 0.05 \mathrm{mag}$ obtained for the same metallicity by Benedict et al. (2011) using HST parallaxes, although it agrees, within the errors, with the value of $M_{V}=0.66 \pm 0.14$ mag derived by Catelan \& Cortes (2008) for RR Lyrae itself $([\mathrm{Fe} / \mathrm{H}]=-1.48 \mathrm{dex})$.

The $M_{V}-[\mathrm{Fe} / \mathrm{H}]$ relations obtained by applying the ABL and Bayesian approaches lead to $M_{V}=0.50 \pm 0.04$ mag and
$M_{V}=0.56_{-0.06}^{+0.04} \mathrm{mag}$ at $[\mathrm{Fe} / \mathrm{H}]=-1.5$ dex, respectively, which are marginally consistent within the relative errors with the absolute magnitude derived by Benedict et al. (2011).

\section{Comparison of results from different relations and conclusions}

In this section we use the TGAS-based $P L, P W, P L Z$, and $M_{V}-$ $[\mathrm{Fe} / \mathrm{H}]$ relations of classical Cepheids, Type II Cepheids, and RR Lyrae stars derived with the three alternative fitting approaches presented in the previous sections to infer the distance to the LMC. We considered only the $P M_{K_{\mathrm{s}}}, P W(V, I)$, and $P W\left(V, K_{\mathrm{s}}\right)$ relations for the classical Cepheids and only the $P M_{K_{\mathrm{s}}}, P M_{K_{\mathrm{s}}} Z$, and $M_{V}-[\mathrm{Fe} / \mathrm{H}]$ relations for the RR Lyrae stars because they are the most relevant in distance scale studies. This comparison may provide the user with the potential and expected levels of systematics of the TGAS parallaxes for these primary standard candles of the cosmological distance ladder. It also gives some perspective on the effects of handling parallaxes in parallax or distance (absolute magnitude) space.

The LMC is a fundamental anchor of the extragalactic distance ladder and is its first step. Over the last two decades the distance modulus of the LMC has been measured countless times using different Population I and II distance indicators and many independent techniques (see e.g. Gibson 2000; Benedict et al. 2002; Clementini et al. 2003; Schaefer 2008, and a recent compilation of literature values by de Grijs et al. 2014), most of which are now converging on a median value of $\mu_{\mathrm{LMC}}=$ $18.49 \pm 0.09 \mathrm{mag}$, which is in close agreement with the value of $(m-M)_{0}=18.493 \pm 0.008$ (stat) \pm 0.047 (syst) mag derived by Pietrzynski et al. (2013) using eight eclipsing binaries in the LMC bar.

The distance moduli derived for the LMC from some of the various relations obtained in this paper are summarised in Table 6, with results for classical Cepheids, Type II Cepheids, and RR Lyrae stars shown in the upper, middle, and lower portions of the table, respectively. They were calculated according to the following procedure: for the classical Cepheids we adopted the $P M_{K_{\mathrm{s}}}$ and $P W(V, I)$ relations derived for the LMC variables by Fouqué et al. (2007) (rows 4 and 2 from the bottom of their Table 8$)$ and the $P W\left(V, K_{\mathrm{s}}\right)$ relation by Ripepi et al. (2012) (their Table 4). For the Type II Cepheids we used the LMC relations in Table 5 of Ripepi et al. (2015). Finally, for 


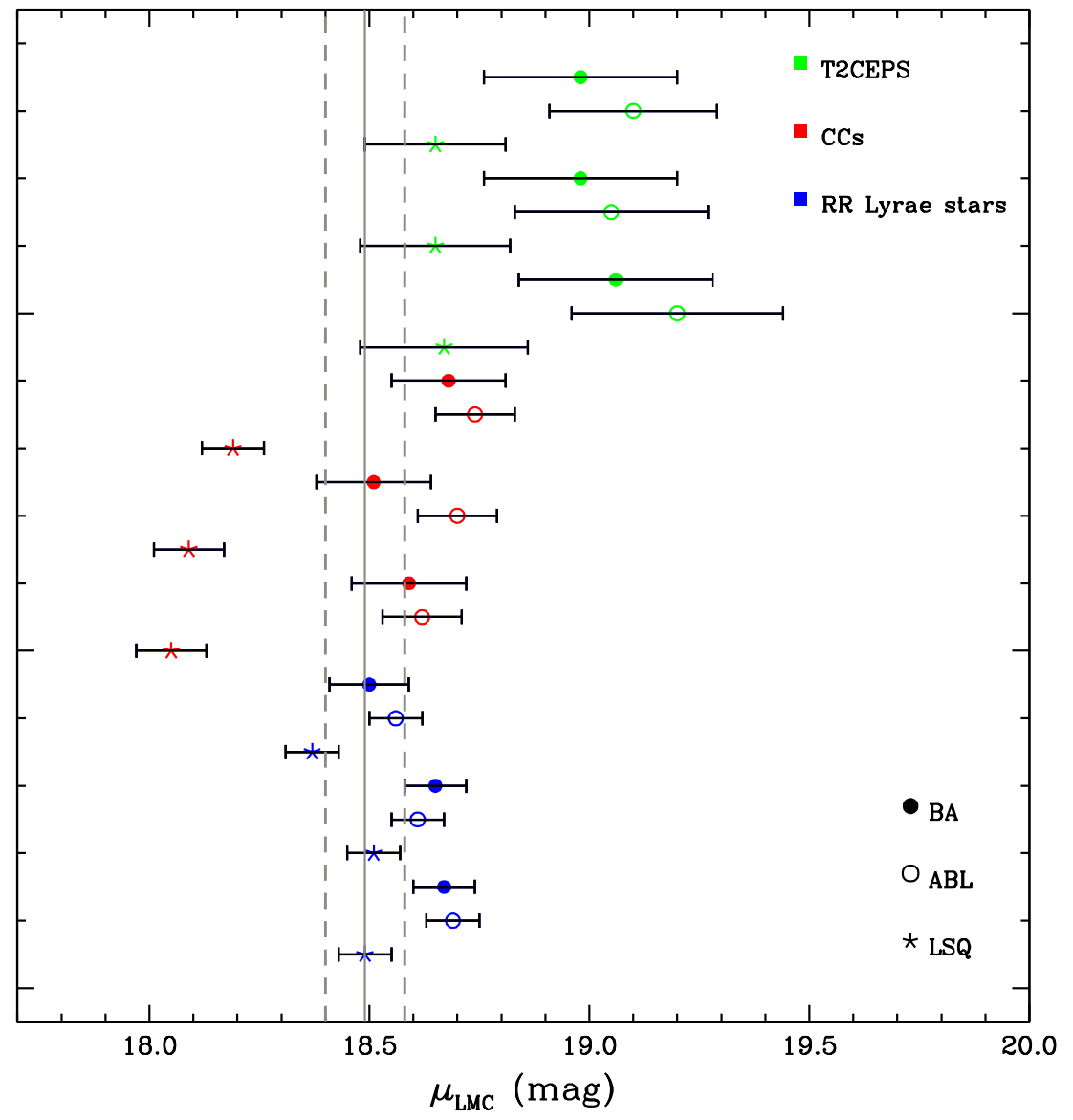

Fig. 25. Values of the LMC distance modulus obtained from the $P L, P L Z$, and $M_{V}-[\mathrm{Fe} / \mathrm{H}]$ relations for RR Lyrae stars (blue symbols), and some of the different TGAS-based $P L$ and $P W$ relations for classical (CCs; red symbols) and Type II Cepheids (T2CEPs; green symbols) derived in this paper. Asterisks, open circles, and filled circles indicate results obtained using the linear least-squares fit (LSQ), the ABL method, and the Bayesian approach (BA), respectively. From bottom to top, RR Lyrae stars (blue symbols): $P M_{K_{\varsigma}}$ relations for 195/200/200 stars (LSQ/ABL/BA methods, respectively) and slope from Muraveva et al. (2015); $P M_{K_{\mathrm{s}}} Z$ relations for $195 / 200 / 200$ stars and slope of the dependence on period from Muraveva et al. (2015); $M_{V}-[\mathrm{Fe} / \mathrm{H}]$ relations for $195 / 200 / 200$ stars with slope from Clementini et al. (2003). For classical Cepheids (red symbols): $P M_{K_{\mathrm{s}}}$ and $P W(V, V-$ I) relations for $95 / 102 / 102$ stars with slopes from Fouqué et al. (2007); and $P W\left(K_{\mathrm{s}}, V-K_{\mathrm{s}}\right)$ relation for 95/102/102 stars with slope from Ripepi et al. (2012). For Type II Cepheids (green symbols): $P M_{J}, P M_{K_{\mathrm{s}}}$ and $P W\left(K_{\mathrm{s}}, J-K_{\mathrm{s}}\right)$ relations for $22 / 26 / 26$ stars with slopes from Ripepi et al. (2015). the RR Lyrae stars we applied the relations from Muraveva et al. (2015) and Clementini et al. (2003). We then subtracted our corresponding TGAS-based zero points from the zero points of these relations, which are in apparent magnitude. Finally, errors on the distance moduli were calculated as the rms of our relations divided by the square root of the number of sources used in the fit. Different values are graphically compared in Fig. 25, where we adopt as a reference value the LMC distance modulus from Pietrzynski et al. (2013).

We find that there is a good consistency, within the errors, between results obtained with the ABL and Bayesian approaches for all three types of variables. On the other hand, the LSQ fit provides systematically shorter moduli. This discrepancy is largest for the classical Cepheids (about 0.5-0.6 mag on average), decreases for the Type II Cepheids (to 0.4-0.5 mag), and is the smallest for the RR Lyrae stars $(0.2 \mathrm{mag})$. When compared with the LMC distance modulus of Pietrzynski et al. (2013), results from the Bayesian approach applied to classical Cepheids are always consistent, within the errors, with the canonical value; the ABL results yield slightly longer distances than Pietrzynski et al. (2013), whereas results from the LSQ fit are 0.2-0.4 mag shorter than currently accepted in the literature. For the Type II Cepheids, all three methods provide longer moduli than the canonical value, by $0.3-0.5 \mathrm{mag}$ for the ABL and Bayesian approaches and by $0.2 \mathrm{mag}$ for the LSQ fit, whose results, hence are still consistent, within the errors, with the Pietrzynski et al. (2013) estimate. There are two times the number of RR Lyrae stars used in this exercise than the classical Cepheids and over seven times more than the Type II Cepheids. The results obtained for the RR Lyrae stars show a much better agreement among the three methods and also a reasonably good agreement with the literature, once again confirming the impressive improvement in quality and statistics of the TGAS parallaxes for RR Lyrae stars compared to HIPPARCOS.

However, taken at face value the results summarised in Table 6 and Fig. 25 span an uncomfortably wide range of over one magnitude around the commonly accepted value of $18.5 \mathrm{mag}$ for the distance modulus of the LMC. Because errors are still fairly large, it is not clear at this stage whether this hints to some systematics in the parallax derivation that may affect the different types of variables used in this paper in different ways. For instance, since no chromatic corrections were applied to derive the TGAS parallaxes, a colour effect could affect more classical Cepheids, which are intrinsically redder and undergo more reddening than RR Lyrae stars or Type II Cepheids. Nevertheless, it is not easy to interpret these results without considering the still very large uncertainties affecting the TGAS parallaxes and, perhaps, the relatively small sample of variable stars that could be used, for instance in the analysis of the Type II Cepheids. We also note that the complexity in the censorships of the samples available prevented us from producing a more reliable estimation of the possible biases introduced by them. As such the results presented in this paper should be considered as preliminary; they will be superseded by results from further releases of Gaia data, allowing the use of samples with more accurate parallaxes and less affected by uncontrolled censorship effects.

Acknowledgements. This work has made use of results from the European Space Agency (ESA) space mission Gaia, the data from which were processed by the Gaia Data Processing and Analysis Consortium (DPAC). Funding for the DPAC has been provided by national institutions, in particular the institutions 
participating in the Gaia Multilateral Agreement. The Gaia mission website is http: //www . cosmos. esa.int/gaia. The authors are current or past member of the ESA and Airbus DS Gaia mission teams and of the Gaia DPAC. This research has made use of the SIMBAD database, operated at CDS, Strasbourg, France. We thank the referee, Pierre Kervella, for his detailed comments and suggestions that have helped to improve the paper analysis and presentation. This work has financially been supported by: the Agenzia Spaziale Italiana (ASI) through grants I/037/08/0, I/058/10/0, 2014-025-R.0, and 2014-025-R.1.2015 to INAF and contracts I/008/10/0 and 2013/030/I.0 to ALTEC S.p.A.; the Algerian Centre de Recherche en Astronomie, Astrophysique et Géophysique of Bouzareah Observatory; the Austrian FWF Hertha Firnberg Programme through grants T359, P20046, and P23737; the BELgian federal Science Policy Office (BELSPO) through various PROgramme de Développement d'Expériences scientifiques (PRODEX) grants; the Brazil-France exchange programmes FAPESP COFECUB and CAPES-COFECUB; the Chinese National Science Foundation through grant NSFC 11573054; the Czech-Republic Ministry of Education, Youth, and Sports through grant LG 15010; the Danish Ministry of Science; the Estonian Ministry of Education and Research through grant IUT401; the European Commission's Sixth Framework Programme through the European Leadership in Space Astrometry (ELSA) Marie Curie Research Training Network (MRTN-CT-2006-033481), through Marie Curie project PIOF-GA 2009-255267 (SAS-RRL), and through a Marie Curie Transfer-of-Knowledge (ToK) fellowship (MTKD-CT-2004-014188); the European Commission's Seventh Framework Programme through grant FP7-606740 (FP7-SPACE-2013-1) for the Gaia European Network for Improved data User Services (GENIUS) and through grant 264895 for the Gaia Research for European Astronomy Train ing (GREAT-ITN) network; the European Research Council (ERC) through grant 320360 and through the European Union's Horizon 2020 research and innovation programme through grant agreement 670519 (Mixing and Angular Momentum tranSport of massIvE stars - MAMSIE); the European Science Foundation (ESF), in the framework of the Gaia Research for European Astronomy Training Research Network Programme (GREAT-ESF); the European Space Agency in the framework of the Gaia project; the European Space Agency Plan for European Cooperating States (PECS) programme through grants for Slovenia; the Czech Space Office through ESA PECS contrac 98058; the Academy of Finland; the Magnus Ehrnrooth Foundation; the French Centre National de la Recherche Scientifique (CNRS) through action "Défi MASTODONS"; the French Centre National d'Études Spatiales (CNES); the French L'Agence Nationale de la Recherche (ANR) "investissements d'avenir" Initiatives D'EXcellence (IDEX) programme PSL* through grant ANR-10IDEX-0001-02; the Région Aquitaine; the Universite de Bordeaux; the French Utinam Institute of the Université de Franche-Comté, supported by the Région de Franche-Comté and the Institut des Sciences de l'Univers (INSU) the German Aerospace Agency (Deutsches Zentrum für Luft- und Raumfahrt e.V., DLR) through grants 50QG0501, 50QG0601, 50QG0602, 50QG0701, 50QG0901, 50QG1001, 50QG1101, 50QG140, 50QG1401, 50QG1402, and 50QG1404; the Hungarian Academy of Sciences through Lendület Programme LP2014-17; the Hungarian National Research, Development, and Innovation Office through grants NKFIH K-115709, K-119517 and PD-116175; the Israel Ministry of Science and Technology through grant 3-9082; the Italian Istituto Nazionale di Astrofisica (INAF); the Netherlands Organisation for Scientific Research (NWO) through grant NWO-M-614.061.414 and through a VICI grant to A. Helmi; the Netherlands Research School for Astronomy (NOVA); the Polish National Science Centre through HARMONIA gran 2015/18/M/ST9/00544; the Portugese Fundação para a Ciência e a Tec nologia (FCT) through grants PTDC/CTE-SPA/118692/2010, PDCTE/CTE AST/81711/2003, and SFRH/BPD/74697/2010; the Strategic Programmes PEstOE/AMB/UI4006/2011 for SIM, UID/FIS/00099/2013 for CENTRA, an UID/EEA/00066/2013 for UNINOVA; the Slovenian Research Agency; the Spanish Ministry of Economy MINECO-FEDER through grants AyA201455216, AyA2011-24052, ESP2013-48318-C2-R, and ESP2014-55996-C2-R and MDM-2014-0369 of ICCUB (Unidad de Excelencia María de Maeztu); the Swedish National Space Board (SNSB/Rymdstyrelsen); the Swiss State Secretariat for Education, Research, and Innovation through the ESA PRODEX programme; the Swiss Mesures d'Accompagnement; the Swiss Activités Nationales Complémentaires; the Swiss National Science Foundation, including an Early Postdoc.Mobility fellowship; the United Kingdom Rutherford Appleton Laboratory; the United Kingdom Science and Technology Facilities Counci (STFC) through grants PP/C506756/1 and ST/I00047X/1; and the United King dom Space Agency (UKSA) through grants ST/K000578/1 and ST/N000978/1.

\section{References}

Anderson, T. W., \& Darling, D. A. 1952, Ann. Math. Statist., 2, 193 Anderson, R. I., Casertano, S., Riess, A. G., et al. 2016, ApJS, 226, 18 Arenou, F., \& Luri, X. 1999, ASP Conf. Ser., 167, 13 Arenou, F., Luri, X., Babusiaux, S., et al. 2017, A\&A, 599, A50 (Gaia SI)
Bailer-Jones, C. A. L. 2015, PASP, 129, 994

Beaton, R. L., Freedman, W. L., Madore, B. F., et al. 2016, ApJ, 832, 210 Benedict, G. F., McArthur, B. E., Fredrick, L. W., et al. 2002, AJ, 123, 473 Benedict, G. F., McArthur, B. E., Feast, M. W., et al. 2007, AJ, 133, 1810 Benedict, G. F., McArthur, B. E., Feast, M. W., et al. 2011, AJ, 142, 187 Berdnikov, L. N. 2008, VizieR Online Data Catalog: II/285 Berdnikov, L. N., Dambis, A. K., \& Vozyakova, O. V. 2000, A\&AS, 143, 211 Bono, G., Caputo, F., \& Santolamazza, P. 1997, A\&A, 317, 171 Bono, G., Castellani, V., \& Marconi, M. 2000, ApJ, 532, L129 Bono, G., Caputo, F., Castellani, V., Marconi, M., \& Storm, J. 2001, MNRAS, 326, 1183

Bono, G., Castellani, V., \& Marconi, M. 2002, ApJ, 565, L83

Bono, G., Caputo, F., Castellani, V., et al. 2003, MNRAS, 344, 1097

Bono, G., Caputo, F., Marconi, M., \& Musella, I. 2010, ApJ, 715, 277

Borissova, J., Rejkuba, M., Minniti, D., Catelan, M., \& Ivanov, V. D. 2009, A\&A, 502, 505

Breitfelder, J., Mérand, A., Kervella, P., et al. 2016, A\&A, 587, A117

Cacciari, C., Clementini, G., \& Fernley, J. A. 1992, ApJ, 396, 219

Cacciari, C., \& Clementini, G. 2003, in Stellar Candles for the Extragalactic Distance Scale, eds. D. Alloin, \& W. Gieren (Berlin: Springer Verlag), Lect. Notes Phys., 635, 105

Caputo, F., Marconi, M., \& Musella, I. 2000a, A\&A, 354, 610

Caputo, F., Castellani, V., Marconi, M., \& Ripepi, V. 2000b, MNRAS, 316, 819

Cardelli, J. A., Clayton, C., \& Mathis, J. S. 1989, AJ, 345, 245

Casertano, S., Riess, A. G., Anderson, J., et al. 2016, ApJ, 825, 11

Casertano, S., Riess, A. G., Bucciarelli, B., \& Lattanzi, M. G. 2017, A\&A, 599, 67

Cassisi, S., Castellani, V., degl'Innocenti, S., \& Weiss, A. 1998, A\&AS, 129, 267

Catelan, M., \& Cortes, C. 2008, ApJ, 676, L135

Catelan, M., Pritzl, B. J., \& Smith, H. A. E. 2004, ApJS, 154, 633

Cioni, M.-R. L., Clementini, G., Girardi, L., et al. 2011, A\&A, 527, A116

Clementini, G., Gratton, R. G., Bragaglia, A., et al. 2003, AJ, 125, 1309

Clementini, G., Ripepi, V., Leccia, S., et al. 2016, A\&A, 595, A133 (Gaia SI)

Coppola, G., Dall'Ora, M., Ripepi, V., et al. 2011, MNRAS, 416, 1056

Cutri, R., Skrutskie, M. F., van Dyk, S., et al. 2003, VizieR Online Data Catalog: II $/ 246$

Dall'Ora, M., Storm, J., Bono, G., et al. 2004, ApJ, 610, 269

Dambis, A. K., Berdnikov, L. N., Kniazev, A. Y., et al. 2013, MNRAS, 435, 3206

Dambis, A. K., Rastorguev, A. S., \& Zabolotskikh, M. V. 2014, MNRAS, 439, 3765

de Bruijne, J., Perryman, M., Lindegren, L., et al. 2005, Gaia Data Processing and Analysis Consortium (DPAC) technical note GAIA-JDB-022

De Grijs, R., Wicker J. E., \& Bono, G., 2014, AJ, 147, 122

Del Principe, M., Piersimoni, A. M., Storm, J., et al. 2006, AJ, 652, 362

Di Fabrizio, L., Clementini, G., Marconi, M., et al. 2002, MNRAS, 336, 841

Drimmel, R., Cabrera-Lavers, A., \& Loṕez-Corredoira, M. 2003, A\&A, 409, 205

ESA 1997, The HIPPARCOS and TYCHO catalogues. Astrometric and photometric star catalogues derived from the ESA HIPPARCOS Space Astrometry Mission, ESA SP, 1200

Evans, N. R. 1994, ApJ, 436, 273

Feast, M. W., Clifton, D. L., Kinman, T. D., van Leeuwen, F., \& Whitelock, P. A 2008, MNRAS, 386, 2115

Fernie, J. D. 2000, AJ, 120, 978

Fernie, J. D., Beattie, B., Evans, N. R., \& Seager, S. 1995, IBVS, 4148

Fernley, J. A. 1994, A\&A, 284, L16

Fiorentino, G., Musella, I., \& Marconi, M. 2013, MNRAS, 434, 2866

Fouqué, P., Arriagada, P., Storm, J., et al. 2007, A\&A, 476, 73

Freedman, W. L., Madore, B. F., Gibson, B. K., et al. 2001, ApJ, 553, 47

Gaia Collaboration (Brown, A.G.A., et al.) 2016a, A\&A, 595, A2

Gaia Collaboration (Prusti, T., et al.) 2016b, A\&A, 595, A1

Gallenne, A., Kervella, P., Mérand, A., et al. 2012, A\&A, 541, A87

Genovali, K., Lemasle, B., Bono, G., et al. 2014, A\&A, 566, A37

Gibson, B. K. 2000, Mem. Soc. Astron. Ital., 71, 693

Gieren, W., Górski, M., Pietrzyński, G., et al. 2013, ApJ, 773, 69

Gingold, R. A. 1985, Mem. Soc. Astron. Ital., 56, 169

Gratton, R. G., Bragaglia, A., Clementini, G., et al. 2004, A\&A, 421, 937

Groenewegen, M. A. T. 1999, A\&AS, 139, 245

Groenewegen, M. A. T., Udalski, A., \& Bono, G. 2008, A\&A, 481, 441

Harris, H. C. 1985, AJ, 90, 756

Høg, E., Fabricius, C., Makarov, V. V., et al. 2000, A\&A, 355, L27

Inno, L., Matsunaga, N., Bono, G., et al. 2013, ApJ, 764, 84

Inno, L., Matsunaga, N., Romaniello, M., et al. 2015, A\&A, 576, A30

Jordi, C., Gebran, M., Carrasco, J. M., et al. 2010, A\&A, 523, A48

Keller, S. C., \& Wood, P. R. 2002, ApJ, 578, 144 
Keller, S. C., \& Wood, P. R. 2006, ApJ, 642, 834

Kervella, P., Mérand, A., Szabados, L., et al. 2008, A\&A, 480, 167

Kervella, P., Bond, H. E., Cracraft, M., et al. 2014, A\&A, 572, A7

Klagyivik, P., \& Szabados, L. 2009, A\&A, 504, 959

Klein, C. R., Richards, J. W., Butler, N. R., \& Bloom J. S., 2014, MNRAS, 440, L96

Kubiak, M., \& Udalski, A. 2003, Acta Astron., 53, 117

Laney, C. D., \& Stobie, R. S. 1992, A\&AS, 93, 93

Lee, Y.-W., Demarque, P., \& Zinn, R. 1990, ApJ, 350, 155

Lemasle, B., François, P., Bono, G., et al. 2007, A\&A, 467, 283

Lemasle, B., François, P., Piersimoni, A., et al. 2008, A\&A, 490, 613

Lemasle, B., Kovtyukh, V., Bono, G., et al. 2015, A\&A, 579, A47

Lindegren, L., Lammers, U., Bastian, U., et al. 2016, A\&A, 595, A4

Longmore, A. J., Fernley, J. A., \& Jameson, R. F. 1986, MNRAS, 220, 279

Longmore, A. J., Dixon, R., Skillen, I., Jameson, R. F., \& Fernley, J. A. 1990, MNRAS, 247, 684

Luck, R. E., \& Lambert, D. L. 2011, AJ, 142, 136

Luck, R. E., Andrievsky, S. M., Kovtyukh, V. V., Gieren, W., \& Graczyk, D. 2011, AJ, 142, 51

Lutz, T. E., \& Kelker, D. H. 1973, PASP, 85, 573

Madore, B. F. 1982, ApJ, 253, 575

Madore, B. F., \& Freedman, W. L. 1991, PASP, 103, 933

Madore, B. F., Hoffman D., Freedman W. L., et al. 2013, ApJ, 776, 135

Maintz, G. 2005, A\&A, 442, 381

Malmquist, K. G. 1936, Stockholms Ob. Medd., 26

Marconi, M. 2015, Mem. Soc. Astron. Ital., 86, 190

Marconi, M., \& Clementini, G. 2005, AJ, 129, 2257

Marconi, M., \& Degl'Innocenti, S. 2007, A\&A, 474, 557

Marconi, M., \& Di Criscienzo, M. 2007, A\&A, 467, 223

Marconi, M., Musella, I., \& Fiorentino, G. 2005, ApJ, 632, 590

Marconi, M., Molinaro, R., Bono, G., et al. 2013a, ApJ, 768, L6

Marconi, M., Molinaro, R., Ripepi, V., Musella, I., \& Brocato, E. 2013b, MNRAS, 428, 2185

Matsunaga, N., Fukushi, H., Nakada, Y., et al. 2006, MNRAS, 370, 1979

Matsunaga, N., Feast, M. W., \& Soszyński, I. 2011, MNRAS, 413, 223

McNamara, D. H., Clementini, G., \& Marconi, M. 2007, AJ, 133, 2752

Mérand, A., Kervella, P., Breitfelder, J., et al. 2015, A\&A, 584, A80

Michalik, D., Lindegren, L., \& Hobbs, D., 2015, A\&A, 574, A115

Molnár, L., \& Szabados, L. 2014, MNRAS, 442, 3222

Monson, A. J., \& Pierce, M. J. 2011, ApJS, 193, 12

Muraveva, T., Palmer, M., Clementini, G., et al. 2015, ApJ, 807, 127

Natale, G., Marconi, M., \& Bono, G. 2008, ApJ, 674, L93

Neeley, J. R., Marengo, M., Bono, G., et al. 2015, ApJ, 808, 11

Nemec, J. M., Nemec, A. F. L., \& Lutz, T. E. 1994, AJ, 108, 222

Ngeow, C.-C. 2012, ApJ, 747, 50

Ngeow, C., \& Kanbur, S. M. 2006, ApJ, 650, 180

Ngeow, C.-C., Kanbur, S. M., Bellinger, E. P., et al. 2012, Ap\&SS, 341, 105

Pejcha, O., \& Kochanek, C. S. 2012, ApJ, 748, 107

Pietrzynski, G., Graczyk, D., Gieren, W., et al. 2013, Nature, 495, 76

Pojmanski, G. 2002, Acta Astron., 52, 397

Pritzl, B. J., Smith, H. A., Stetson, P. B., et al. 2003, AJ, 126, 1381

Riess, A. G., Macri, L., Casertano, S., et al. 2011, ApJ, 730, 119

Riess, A. G., Casertano, S., Anderson, J., MacKenty, J., \& Filippenko, A. V. 2014, ApJ, 785, 161

Riess, A. G., Macri, L. M., Hoffmann, S. L., et al. 2016, ApJ, 826, 56

Ripepi, V., Moretti, M. I., Marconi, M., et al. 2012, MNRAS, 424, 1807

Ripepi, V., Moretti, M.-I., Marconi, M., et al. 2015, MNRAS, 446, 3034

Ripepi, V., Marconi, M., Moretti, M. I., et al. 2016, ApJS, 224, 21

Romaniello, M., Primas, F., Mottini, M., et al. 2008, A\&A, 488, 731

Saha, A., Thim, F., Tammann, G. A., Reindl, B., \& Sandage, A. 2006, ApJS 165,108

Samus, N. N., Durlevich O. V., Goranskij V. P., et al. 2007-2015, General Catalogue of Variable Stars (Samus+ 2007-2015)

Samus', N. N., Kazarovets, E. V., Durlevich, O. V., Kireeva, N. N., \& Pastukhova, E. N. 2017, Astron. Rep., 61, 80

Schaefer, B. E. 2008, AJ, 135, 112

Schlafly, E. F., \& Finkbeiner, D. P. 2011, ApJ, 737, 103

Sesar, B., Fouesneau, M., Price-Whelan, A. M., et al. 2017, ApJ, 838, 107

Skillen, I., Fernley, J. A., Stobie, R. S., \& Jameson, R. F. 1993, MNRAS, 265, 301

Sollima, A., Cacciari, C., \& Valenti, E., 2006, MNRAS, 372, 1675

Sollima, A., Cacciari, C., Arkharov, A. A. H., et al. 2008, MNRAS, 384, 1583

Soszyński, I., Udalski, A., Szymański, M. K., et al. 2008, Acta Astron., 58, 293

Szabados, L. 1997, in HipPARcos - Venice 97", ESA SP, 402, 657

Szabados, L. 2003, IBVS, 5394

Sziládi, K., Vinkó, J., Poretti, E., Szabados, L., \& Kun, M. 2007, A\&A, 473, 579
Tammann, G. A., Sandage, A., \& Reindl, B. 2003, A\&A, 404, 423

Turner, D. G., Billings, G. W., \& Berdnikov, L. N. 2001, PASP, 113, 715

Udalski, A., M., Kaluzny, J., Kubiak, M., \& Mateo, M. 1992, Acta Astron., 42, 253

Udalski, A., Soszynski, I., Szymanski, M., et al. 1999, Acta Astron., 49, 45 van Leeuwen, F. 2007a, HIPPARcos, the New Reduction of the Raw Data, Astrophys. Space Sci. Lib., 350

van Leeuwen, F. 2007b, A\&A, 474, 653

Wade, R. A., Donley, J., Fried, R., White, R. E., \& Saha, A. 1999, AJ, 118, 2442

Wallerstein, G. 2002, PASP, 114, 689

Wallerstein, G., \& Cox, A. N. 1984, PASP, 96, 677

Welch, D. L., Wieland, F., McAlary, C. W., et al. 1984, ApJS, 54, 547

Wenger, M., Ochsenbein, F., Egret, D., et al. 2000, A\&AS, 143, 9

Wood, P. R. S., Arnold, A., \& Sebo, K. M. 1997, ApJ, 485, L25

Zinn, R., \& West, M. J. 1984, ApJS, 55, 45

1 INAF-Osservatorio Astronomico di Bologna, via Piero Gobetti 93/3, 40129 Bologna, Italy

2 Department of Astronomy, University of Geneva, Chemin des Maillettes 51, 1290 Versoix, Switzerland

3 INAF-Osservatorio Astronomico di Capodimonte, via Moiariello 16, 80131 Napoli, Italy

4 Dipartimento di Fisica e Astronomia, Università di Bologna, via Piero Gobetti 93/2, 40129 Bologna, Italy

5 Dpto. de Inteligencia Artificial, UNED, c/ Juan del Rosal 16, 28040 Madrid, Spain

6 Institut de Ciències del Cosmos, Universitat de Barcelona (IEECUB), Martí Franquès 1, 08028 Barcelona, Spain

7 Department of Astronomy, University of Geneva, Chemin d'Ecogia 16, 1290 Versoix, Switzerland

8 Konkoly Observatory, Research Centre for Astronomy and Earth Sciences, Hungarian Academy of Sciences, Konkoly Thege Miklós út 15-17, 1121 Budapest, Hungary

9 Department of Physics and Astronomy, The Johns Hopkins University, 3400 N Charles St., Baltimore, MD 21218, USA

10 Scientific Support Office, Directorate of Science, European Space Research and Technology Centre (ESA/ESTEC), Keplerlaan 1, 2201AZ, Noordwijk, The Netherlands

11 Leiden Observatory, Leiden University, Niels Bohrweg 2, 2333 CA Leiden, The Netherlands

12 INAF-Osservatorio astronomico di Padova, Vicolo Osservatorio 5, 35122 Padova, Italy

13 GEPI, Observatoire de Paris, PSL Research University, CNRS, Univ. Paris Diderot, Sorbonne Paris Cité, 5 Place Jules Janssen, 92190 Meudon, France

14 Max Planck Institute for Astronomy, Königstuhl 17, 69117 Heidelberg, Germany

15 Astronomisches Rechen-Institut, Zentrum für Astronomie der Universität Heidelberg, Mönchhofstr. 12-14, 69120 Heidelberg, Germany

16 Institute of Astronomy, University of Cambridge, Madingley Road, Cambridge CB3 OHA, UK

17 Mission Operations Division, Operations Department, Directorate of Science, European Space Research and Technology Centre (ESA/ESTEC), Keplerlaan 1, 2201 AZ, Noordwijk, The Netherlands

18 Lohrmann Observatory, Technische Universität Dresden, Mommsenstraße 13, 01062 Dresden, Germany

19 European Space Astronomy Centre (ESA/ESAC), Camino bajo del Castillo, s/n, Urbanizacion Villafranca del Castillo, Villanueva de la Cañada, 28692 Madrid, Spain

20 Lund Observatory, Department of Astronomy and Theoretical Physics, Lund University, Box 43, 22100 Lund, Sweden

21 Laboratoire Lagrange, Université Nice Sophia-Antipolis, Observatoire de la Côte d'Azur, CNRS, CS 34229, 06304 Nice Cedex, France

22 CNES Centre Spatial de Toulouse, 18 avenue Edouard Belin, 31401 Toulouse Cedex 9, France 
23 Institut d'Astronomie et d'Astrophysique, Université Libre de Bruxelles CP 226, Boulevard du Triomphe, 1050 Brussels, Belgium

24 F.R.S.-FNRS, Rue d'Egmont 5, 1000 Brussels, Belgium

25 INAF-Osservatorio Astrofisico di Arcetri, Largo Enrico Fermi 5, 50125 Firenze, Italy

26 Telespazio Vega UK Ltd for ESA/ESAC, Camino bajo del Castillo, $\mathrm{s} / \mathrm{n}$, Urbanizacion Villafranca del Castillo, Villanueva de la Cañada, 28692 Madrid, Spain

27 Laboratoire d'astrophysique de Bordeaux, Université de Bordeaux, CNRS, B18N, allée Geoffroy Saint-Hilaire, 33615 Pessac, France

28 Instituut voor Sterrenkunde, KU Leuven, Celestijnenlaan 200D, 3001 Leuven, Belgium

29 Department of Astrophysics/IMAPP, Radboud University Nijmegen, PO Box 9010, 6500 GL Nijmegen, The Netherlands

30 Mullard Space Science Laboratory, University College London, Holmbury St Mary, Dorking, Surrey RH5 6NT, UK

31 INAF-Osservatorio Astrofisico di Torino, via Osservatorio 20, 10025 Pino Torinese (TO), Italy

32 Niels Bohr Institute, University of Copenhagen, Juliane Maries Vej 30, 2100 Copenhagen $\emptyset$, Denmark

33 Centre for Electronic Imaging, Department of Physical Sciences, The Open University, Walton Hall MK7 6AA Milton Keynes, UK

34 ALTEC S.p.a, Corso Marche, 79,10146 Torino, Italy

35 Serco Gestión de Negocios for ESA/ESAC, Camino bajo del Castillo, s/n, Urbanizacion Villafranca del Castillo, Villanueva de la Cañada, 28692 Madrid, Spain

36 STFC, Rutherford Appleton Laboratory, Harwell, Didcot, OX11 OQX, UK

37 Gaia DPAC Project Office, ESAC, Camino bajo del Castillo, s/n, Urbanizacion Villafranca del Castillo, Villanueva de la Cañada, 28692 Madrid, Spain

38 SYRTE, Observatoire de Paris, PSL Research University, CNRS, Sorbonne Universités, UPMC Univ. Paris 06, LNE, 61 avenue de l'Observatoire, 75014 Paris, France

39 National Observatory of Athens, I. Metaxa and Vas. Pavlou, Palaia Penteli, 15236 Athens, Greece

40 IMCCE, Observatoire de Paris, PSL Research University, CNRS, Sorbonne Universités, UPMC Univ. Paris 06, Univ. Lille, 77 av. Denfert-Rochereau, 75014 Paris, France

${ }^{41}$ Royal Observatory of Belgium, Ringlaan 3, 1180 Brussels, Belgium

42 Institut d'Astrophysique Spatiale, Université Paris XI, UMR 8617 , CNRS, Bâtiment 121, 91405 Orsay Cedex, France

43 Institute for Astronomy, Royal Observatory, University of Edinburgh, Blackford Hill, Edinburgh EH9 3HJ, UK

${ }^{44} \mathrm{HE}$ Space Operations BV for ESA/ESAC, Camino bajo del Castillo, s/n, Urbanizacion Villafranca del Castillo, Villanueva de la Cañada, 28692 Madrid, Spain

45 Institut d'Astrophysique et de Géophysique, Université de Liège, $19 \mathrm{c}$, Allée du 6 Août, 4000 Liège, Belgium

46 Área de Lenguajes y Sistemas Informáticos, Universidad Pablo de Olavide, Ctra. de Utrera, km 1. 41013 Sevilla, Spain

47 Observatoire Astronomique de Strasbourg, Université de Strasbourg, CNRS, UMR 7550, 11 rue de l'Université, 67000 Strasbourg, France

48 Kavli Institute for Cosmology, University of Cambridge, Madingley Road, Cambride CB3 OHA, UK

49 Aurora Technology for ESA/ESAC, Camino bajo del Castillo, s/n, Urbanizacion Villafranca del Castillo, Villanueva de la Cañada, 28692 Madrid, Spain

${ }^{50}$ Laboratoire Univers et Particules de Montpellier, Université Montpellier, Place Eugène Bataillon, CC72, 34095 Montpellier Cedex 05, France

51 Department of Astrophysics, Astronomy and Mechanics, National and Kapodistrian University of Athens, Panepistimiopolis, Zografos, 15783 Athens, Greece

52 Department of Physics and Astronomy, Division of Astronomy and Space Physics, Uppsala University, Box 516, 75120 Uppsala, Sweden
53 Università di Catania, Dipartimento di Fisica e Astronomia, Sezione Astrofisica, via S. Sofia 78, 95123 Catania, Italy

54 INAF-Osservatorio Astrofisico di Catania, via S. Sofia 78, 95123 Catania, Italy

55 Universidade da Coruña, Facultade de Informática, Campus de Elviña S/N, 15071 A Coruña, Spain

56 CENTRA, Universidade de Lisboa, FCUL, Campo Grande, Edif. C8, 1749-016 Lisboa, Portugal

57 University of Helsinki, Department of Physics, PO Box 64, 00014 University of Helsinki, Finland

${ }^{58}$ Finnish Geospatial Research Institute FGI, Geodeetinrinne 2, 02430 Masala, Finland

59 Isdefe for ESA/ESAC, Camino bajo del Castillo, s/n, Urbanizacion Villafranca del Castillo, Villanueva de la Cañada, 28692 Madrid, Spain

60 ASI Science Data Center, via del Politecnico SNC, 00133 Roma, Italy

61 Institut UTINAM UMR6213, CNRS, OSU THETA FrancheComté Bourgogne, Université Bourgogne Franche-Comté, 25000 Besançon, France

62 Elecnor Deimos Space for ESA/ESAC, Camino bajo del Castillo, $\mathrm{s} / \mathrm{n}$, Urbanizacion Villafranca del Castillo, Villanueva de la Cañada, 28692 Madrid, Spain

63 Thales Services for CNES Centre Spatial de Toulouse, 18 avenue Edouard Belin, 31401 Toulouse Cedex 9, France

${ }^{64}$ EURIX S.r.l., via Carcano 26, 10153 Torino, Italy

65 University of Vienna, Department of Astrophysics, Türkenschanzstraße 17, A1180 Vienna, Austria

66 ON/MCTI-BR, Rua Gal. José Cristino 77, 20921-400 Rio de Janeiro, RJ, Brazil

67 OV/UFRJ-BR, Ladeira Pedro Antônio 43, 20080-090 Rio de Janeiro, RJ, Brazil

68 Faculdade Ciencias, Universidade do Porto, Departamento Matematica Aplicada, Rua do Campo Alegre, 687 4169-007 Porto, Portugal

69 Instituto de Astrofísica e Ciências do Espaço, Universidade de Lisboa Faculdade de Ciências, Campo Grande, PT1749-016 Lisboa, Portugal

${ }^{70}$ Departamento de Astrofísica, Centro de Astrobiología (CSICINTA), ESA-ESAC. Camino Bajo del Castillo s/n. 28692 Villanueva de la Cañada, Madrid, Spain

71 Department of Physics and Astronomy, University of Leicester, University Road, Leicester LE1 7RH, UK

${ }^{72}$ University of Oviedo, Campus Universitario, 33203 Gijón, Spain

73 University of Cádiz, Avd. De la universidad, Jerez de la Frontera, 11002 Cádiz, Spain

74 Kapteyn Astronomical Institute, University of Groningen, Landleven 12, 9747 AD Groningen, The Netherlands

75 Consorci de Serveis Universitaris de Catalunya, C/ Gran Capità, 2-4 3rd floor, 08034 Barcelona, Spain

76 University of Turin, Department of Computer Sciences, Corso Svizzera 185, 10149 Torino, Italy

77 INAF-Osservatorio Astronomico di Roma, via di Frascati 33, 00078 Monte Porzio Catone (Roma), Italy

78 CRAAG-Centre de Recherche en Astronomie, Astrophysique et Géophysique, Route de l'Observatoire BP 63 Bouzareah, 16340 Algiers, Algeria

79 Universiteit Antwerpen, Onderzoeksgroep Toegepaste Wiskunde, Middelheimlaan 1, 2020 Antwerpen, Belgium

80 Department of Physics and Astronomy, University of Padova, via Marzolo 8, 35131 Padova, Italy

81 INAF-Osservatorio Astronomico di Teramo, via Mentore Maggini, 64100 Teramo, Italy

82 Instituto de Astronomia, Geofîsica e Ciências Atmosféricas, Universidade de São Paulo, Rua do Matão, 1226, Cidade Universitaria, 05508-900 São Paulo, SP, Brazil

83 Department of Geosciences, Tel Aviv University, 6997801 Tel Aviv, Israel

84 Astronomical Institute Anton Pannekoek, University of Amsterdam, PO Box 94249, 1090 GE, Amsterdam, The Netherlands 
85 Leibniz Institute for Astrophysics Potsdam (AIP), An der Sternwarte 16, 14482 Potsdam, Germany

86 ATOS for CNES Centre Spatial de Toulouse, 18 avenue Edouard Belin, 31401 Toulouse Cedex 9, France

87 UNINOVA-CTS, Campus FCT-UNL, Monte da Caparica, 2829516 Caparica, Portugal

88 Laboratoire Géoazur, Université Nice Sophia-Antipolis, UMR 7329, CNRS, Observatoire de la Côte d'Azur, 250 rue A. Einstein, 06560 Valbonne, France

89 RHEA for ESA/ESAC, Camino bajo del Castillo, s/n, Urbanizacion Villafranca del Castillo, Villanueva de la Cañada, 28692 Madrid, Spain

90 Astronomical Institute, Academy of Sciences of the Czech Republic, Fričova 298, 25165 Ondřejov, Czech Republic

91 Barcelona Supercomputing Center - Centro Nacional de Supercomputación, c/ Jordi Girona 29, Ed. Nexus II, 08034 Barcelona, Spain

92 Department of Mechanical Engineering, University of La Rioja, c/ San José de Calasanz, 31, 26004 Logroño, La Rioja, Spain

93 ETSE Telecomunicación, Universidade de Vigo, Campus LagoasMarcosende, 36310 Vigo, Galicia, Spain

94 SRON, Netherlands Institute for Space Research, Sorbonnelaan 2, 3584CA, Utrecht, The Netherlands

95 Faculty of Mathematics and Physics, University of Ljubljana, Jadranska ulica 19, 1000 Ljubljana, Slovenia

96 Physics Department, University of Antwerp, Groenenborgerlaan 171, 2020 Antwerp, Belgium

97 Harvard-Smithsonian Center for Astrophysics, 60 Garden Street, Cambridge MA 02138, USA

98 Institut de Physique de Rennes, Université de Rennes 1, 35042 Rennes, France

99 Shanghai Astronomical Observatory, Chinese Academy of Sciences, 80 Nandan Rd, 200030 Shanghai, PR China

100 CSC Danmark A/S, Retortvej 8, 2500 Valby, Denmark

101 Las Cumbres Observatory Global Telescope Network, Inc., 6740 Cortona Drive, Suite 102, Goleta, CA 93117, USA

102 Astrophysics Research Institute, Liverpool John Moores University, L3 5RF, UK

103 Baja Observatory of University of Szeged, Szegedi út III/70, 6500 Baja, Hungary

104 Laboratoire AIM, IRFU/Service d'Astrophysique - CEA/DSM CNRS - Université Paris Diderot, Bât 709, CEA-Saclay, 91191 Gif-sur-Yvette Cedex, France

105 INAF-Osservatorio Astronomico di Trieste, via G.B. Tiepolo 11, 34143, Trieste, Italy

106 Laboratoire de l'Accélérateur Linéaire, Université Paris-Sud, CNRS/IN2P3, Université Paris-Saclay, 91898 Orsay Cedex, France

107 École polytechnique fédérale de Lausanne, SB MATHAA STAP, MA B1 473 (Bâtiment MA), Station 8, 1015 Lausanne, Switzerland

108 INAF-Istituto di Astrofisica Spaziale e Fisica Cosmica di Bologna, via Piero Gobetti 101, 40129 Bologna, Italy

109 Technical University of Madrid, José Gutiérrez Abascal 2, 28006 Madrid, Spain

110 EQUERT International for CNES Centre Spatial de Toulouse, 18 avenue Edouard Belin, 31401 Toulouse Cedex 9, France

111 AKKA for CNES Centre Spatial de Toulouse, 18 avenue Edouard Belin, 31401 Toulouse Cedex 9, France

112 Villanova University, Dept. of Astrophysics and Planetary Science, 800 E Lancaster Ave, Villanova PA 19085, USA

113 Vitrociset Belgium for ESA/ESAC, Camino bajo del Castillo, s/n, Urbanizacion Villafranca del Castillo, Villanueva de la Cañada, 28692 Madrid, Spain

114 Fork Research, Rua do Cruzado Osberno, Lt. 1, 9 esq., Lisboa, Portugal

115 APAVE SUDEUROPE SAS for CNES Centre Spatial de Toulouse, 18 avenue Edouard Belin, 31401 Toulouse Cedex 9, France

116 Spanish Virtual Observatory, Spain

117 Fundación Galileo Galilei - INAF, Rambla José Ana Fernández Pérez 7, 38712 Breña Baja, Santa Cruz de Tenerife, Spain
118 INSA for ESA/ESAC, Camino bajo del Castillo, s/n, Urbanizacion Villafranca del Castillo, Villanueva de la Cañada, 28692 Madrid, Spain

119 Dpto. Arquitectura de Computadores y Automática, Facultad de Informática, Universidad Complutense de Madrid, C/ Prof. José García Santesmases s/n, 28040 Madrid, Spain

${ }^{120}$ H H Wills Physics Laboratory, University of Bristol, Tyndall Avenue, Bristol BS8 1TL, UK

121 Stellar Astrophysics Centre, Aarhus University, Department of Physics and Astronomy, 120 Ny Munkegade, Building 1520, 8000 Aarhus C, Denmark

122 Applied Physics Department, University of Vigo, 36310 Vigo, Spain

123 HE Space Operations BV for ESA/ESTEC, Keplerlaan 1, 2201AZ, Noordwijk, The Netherlands

124 Warsaw University Observatory, Al. Ujazdowskie 4, 00-478 Warszawa, Poland

125 Instituto de Astrofísica de Canarias, 38205 La Laguna, Tenerife, Spain

126 Universidad de La Laguna, Departamento de Astrofísica, $38206 \mathrm{La}$ Laguna, Tenerife, Spain

127 RHEA for ESA/ESTEC, Keplerlaan 1, 2201AZ, Noordwijk, The Netherlands

128 Max Planck Institute for Solar System Research, Justus-vonLiebig-Weg 3, 37077 Göttingen, Germany

129 SISSA (Scuola Internazionale Superiore di Studi Avanzati), via Bonomea 265, 34136 Trieste, Italy

${ }^{130}$ Instituto Nacional de Pesquisas Espaciais/Ministério da Ciencia Tecnologia, Avenida dos Astronautas 1758, São José Dos Campos, SP 12227-010, Brazil

131 Argelander Institut für Astronomie der Universität Bonn, Auf dem Hügel 71, 53121 Bonn, Germany

132 European Southern Observatory (ESO), Karl-SchwarzschildStraße 2, 85748 Garching bei München, Germany

133 Laboratory of Optics, Lasers and Systems, Faculty of Sciences, University of Lisbon, Campus do Lumiar, Estrada do Paço do Lumiar, 22, 1649-038 Lisboa, Portugal

134 Department of Physics and Astronomy, Notre Dame University, Louaize, PO Box 72, Zouk Mikaël, Lebanon

135 University of Nova Gorica, Vipavska 13, 5000 Nova Gorica, Slovenia

136 Max Planck Institute for Extraterrestrial Physics, OPINAS, Gießenbachstraße, 85741 Garching, Germany

137 NASA/IPAC Infrared Science Archive, California Institute of Technology, Mail Code 100-22, 770 South Wilson Avenue, Pasadena, CA, 91125, USA

138 Center of Applied Space Technology and Microgravity (ZARM), c/o Universität Bremen, Am Fallturm 1, 28359 Bremen, Germany

139 RHEA System for ESA/ESOC, Robert Bosch Straße 5, 64293 Darmstadt, Germany

140 Tartu Observatory, 61602 Tõravere, Estonia

141 Sydney Institute for Astronomy, School of Physics A28, The University of Sydney, NSW 2006, Australia

142 Slovak Organisation for Space Activities, Zamocka 18, 85101 Bratislava, Slovak Republic

143 National Astronomical Observatories, CAS, 100012 Beijing, PR China

144 US Naval Observatory, Astrometry Department, 3450 Massachusetts Ave. NW, Washington DC 20392-5420 D.C., USA

145 European Southern Observatory (ESO), Alonso de Córdova 3107, Vitacura, Casilla 19001, Santiago de Chile, Chile

146 Airbus Defence and Space SAS, 31 Rue des Cosmonautes, 31402 Toulouse Cedex 4, France

147 EJR-Quartz BV for ESA/ESTEC, Keplerlaan 1, 2201AZ, Noordwijk, The Netherlands

148 The Server Labs for ESA/ESAC, Camino bajo del Castillo, s/n, Urbanizacion Villafranca del Castillo, Villanueva de la Cañada, 28692 Madrid, Spain 
149 Astronomical Observatory Institute, Faculty of Physics, A. Mickiewicz University, ul. Słoneczna 36, 60-286 Poznań, Poland

150 CS Systèmes d'Information for CNES Centre Spatial de Toulouse, 18 avenue Edouard Belin, 31401 Toulouse Cedex 9, France

151 Directorate of Science, European Space Research and Technology Centre (ESA/ESTEC), Keplerlaan 1, 2201AZ, Noordwijk, The Netherlands

152 Praesepe BV for ESA/ESAC, Camino bajo del Castillo, s/n, Urbanizacion Villafranca del Castillo, Villanueva de la Cañada, 28692 Madrid, Spain

153 Sorbonne Universités UPMC et CNRS, UMR7095, Institut d'Astrophysique de Paris, 75014, Paris, France

154 GMV for ESA/ESAC, Camino bajo del Castillo, s/n, Urbanizacion Villafranca del Castillo, Villanueva de la Cañada, 28692 Madrid, Spain

155 Institute of Theoretical Physics and Astronomy, Vilnius University, Sauletekio al. 3, 10222 Vilnius, Lithuania

156 S[\&]T Corporation, PO Box 608, 2600 AP, Delft, The Netherlands
157 Department of Space Studies, Southwest Research Institute (SwRI), 1050 Walnut Street, Suite 300, Boulder, Colorado 80302, USA

158 Deutsches Zentrum für Luft- und Raumfahrt, Institute of Space Systems, Am Fallturm 1, 28359 Bremen, Germany

159 University of Applied Sciences Munich, Karlstr. 6, 80333 Munich, Germany

160 Dipartimento di Fisica, Università di Roma Tor Vergata, via della Ricerca Scientifica 1, 00133 Rome, Italy

161 Department of Physics and Astronomy, University of the Western Cape, Robert Sobukwe Road, 7535 Bellville, Cape Town, South Africa

162 INAF-Istituto di Radioastronomia, via Piero Gobetti 101, 40129 Bologna, Italy

163 Department of Physics, Florida International University, 11200 SW 8th Street, Miami, FL 33199, USA

164 Hamburger Sternwarte, Gojenbergsweg 112, 21029 Hamburg, Germany 

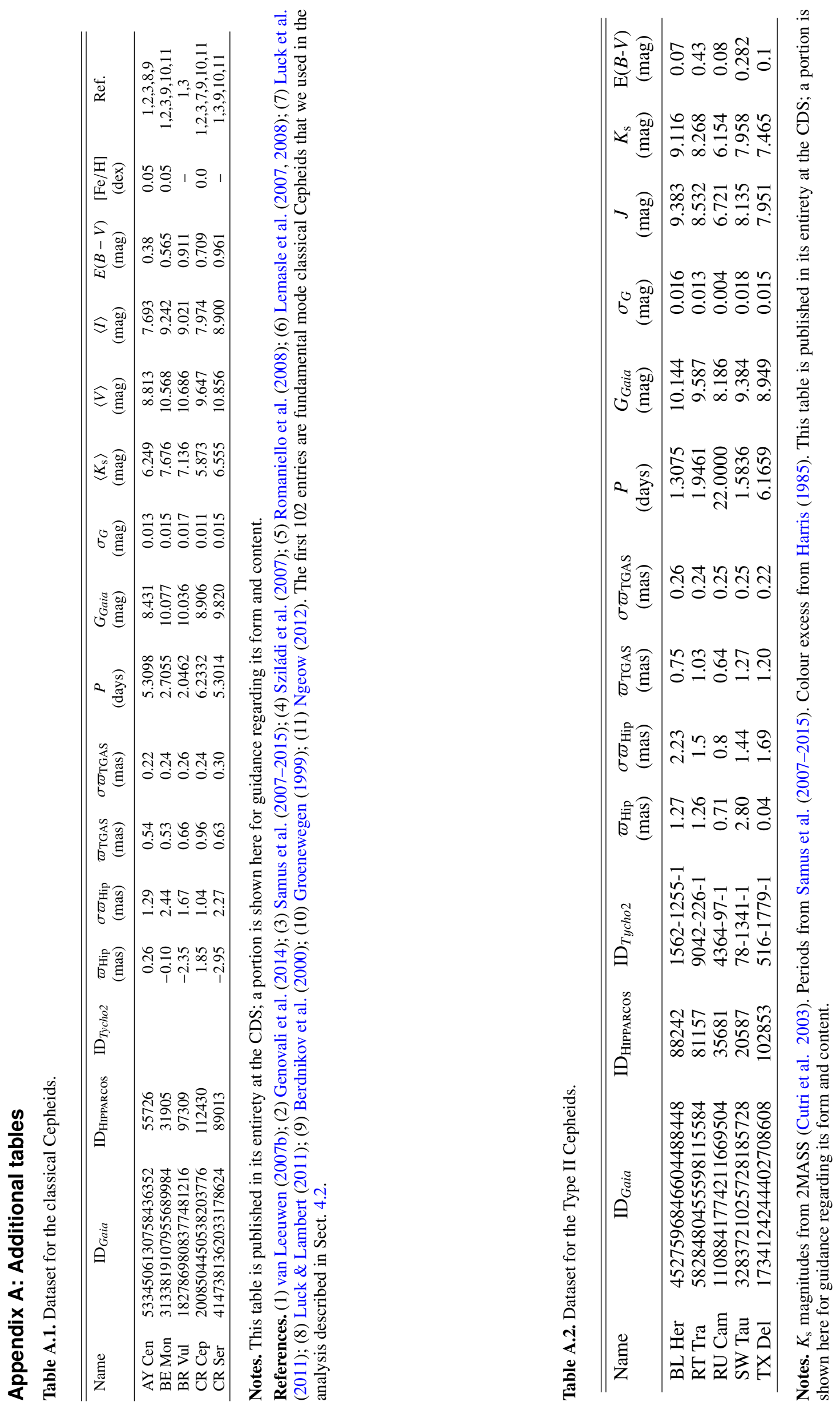
Gaia Collaboration (Clementini, G., et al.): Gaia Data Release 1

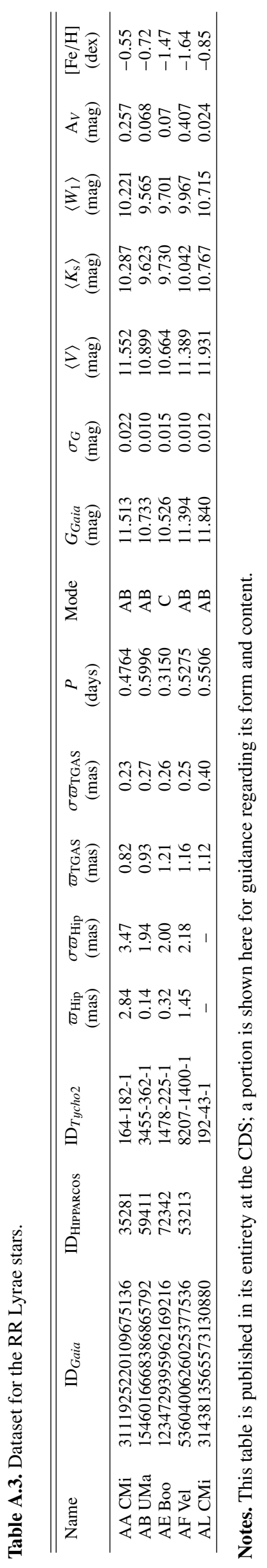

